\title{
How science-policy relations change: implementing EU environmental policy in Poland
}

Katarzyna Kowalczewska 


\section{Thesis committee}

\section{Promotor}

Prof. Dr E. Turnhout

Personal chair at the Forest and Nature Conservation Policy Group

Wageningen University \& Research

\section{Co-promotor}

Dr J.H. Behagel

Assistant professor at the Forest and Nature Conservation Policy Group

Wageningen University \& Research

\section{Other members}

Prof. Dr P.M. Macnaghten, Wageningen University \& Research

Prof. Dr Z. Karaczun, Warsaw University of Life Sciences, Poland

Dr T.A.P. Metze, Wageningen University \& Research

Dr E. Kunseler, Netherlands Environmental Assessment Agency

This research was conducted under the auspices of the Wageningen School of Social Sciences (WASS). 


\title{
How science-policy relations change: implementing EU environmental policy in Poland
}

\author{
Katarzyna Kowalczewska
}

Thesis

submitted in fulfilment of the requirements for the degree of doctor at Wageningen University by the authority of the Rector Magnificus

Prof. Dr A.P.J. Mol, in the presence of the

Thesis Committee appointed by the Academic Board to be defended in public on Monday 27 May 2019 at $13: 30$ in the Aula. 
Katarzyna Kowalczewska

How science-policy relations change: implementing EU environmental policy in Poland, 174 pages.

PhD thesis, Wageningen University, Wageningen, the Netherlands (2019)

With references, with summary in English

ISBN 978-94-6343-961-9

DOI https://doi.org/10.18174/476385 


\section{Preface and Acknowledgements}

After obtaining my Master of Environmental Sciences diploma at Wageningen University I did not expect to obtain a doctoral degree at the same university a few years later. The start of my PhD start did not go very well. There were problems with my accommodation at Wageningen and getting to a mutual agreement on the contract, and my laptop stopped working. Additionally, the perspective of a temporary separation from my newly-married husband who stayed in Warsaw at that time did not help. Esther Turnhout, the promotor of this thesis, came up with a solution that in the end led to the result of this finished PhD thesis: I became an external PhD student of Wageningen University. Moreover, I decide to continue my regular work in Poland at the Ministry of Agriculture and Rural Development. At the time, I had no idea of the enormity of the task ahead nor of the difficulties of the writing process while being alone and more than one thousand kilometres away from my supervisor(s) and the chair group of FNP.

During the many years of writing process, I had ups and downs but never ideas to quit my PhD. My professional experience in policymaking offered me a lot of ideas to write about and explore, but also less time to write it. From time to time, I sent my promotor, Esther, long emails with new ideas and explanations about what I will investigate more in my PhD thesis. She was always supportive even when - reflecting about it today - some of these ideas were too ambitious, not always realistic and too much policy and solution oriented. A breakthrough moment happened when I joined the team at the Embassy of the Kingdom of the Netherlands in Warsaw. Everyday contact with the Dutch, their logical way of thinking, process-orientation, and deliberation style, reminded me of why I wanted to do my PhD thesis in the Netherlands. I decided to complete my PhD within the duration of my first 4-year contract at the Embassy. I think Esther noticed my additional motivation and proposed a co-promotor. Jelle Behagel, whom I already knew, joined my project at the beginning of 2016 and helped a lot towards finalizing my thesis.

The last three years have been a challenge to combine work, home and children with writing a doctoral dissertation and therefore a big sacrifice. Although writing has been an individual process, I would not have competed this without the support of some important people which I would like to thank. I am sincerely grateful to my promotor Professor Esther Turnhout for her intuition and belief in my potential. She provided me with support, theoretical inspirations (although not always understood by me), and, most importantly, constructive feedback in the writing and research processes to deliver 
what was expected. I have learned how to use this feedback by listening and making sense of it in my own thesis. I am also grateful to my co-promotor Dr Jelle Behagel for his supervision in the last three years that was substantial for my writing process. I appreciate the time he devoted to read all texts I sent him and for all the Skype-calls we had. His feedback was always constructive, explaining what can or cannot be done/written instead of saying what was wrong. I did not realize how important it is to keep a positive thinking mood and how motivating this can be within the writing process. Jelle also turned out to be a very nice colleague with whom you can go for a beer and have fun which I also appreciated as it allowed me to develop a more personal contact with him.

During my work at the Polish Ministry of Agriculture and the European Commission I met a few colleagues whose mentoring and advice were very helpful in broadening my knowledge and collecting data for my research. I cannot mention them here all by name, but I am grateful for their willingness to share and the simple fact that I could work with them which was a great learning experience for me. I am also grateful to all my colleagues at the Embassy of the Kingdom of the Netherlands in Warsaw for understanding my ambition. I thank Martijn Homan in particular for the support I received during the last phase of writing my dissertation.

I also thank my three Dutch girlfriends Cathelijne, Mariola, and Ilse, for always being there for me and with me in the Netherlands in good and bad moments.

Finally, I would like to thank my family who always supported my choices and had an unconditional confidence in me. Without my parents and my parents in law who many times took care of my kids I could not have free Saturdays and Sundays to write. Your everyday involvement in being great grandparents is very much appreciated. Finally, the biggest thanks go to Tomasz, my husband, for his patience, support, keeping family away in weekends, taking care of home, and assisting me wherever I needed it. You have read many pieces of this PhD thesis and helped me with the final shape of it. Thanks for being always there for me.

It is another Sunday, when I am writing this preface and acknowledgements. Now, I really look forward to free weekends and holidays without the laptop, with Tomasz and the kids.

Katarzyna Kowalczewska 


\section{TABLE OF CONTENTS}

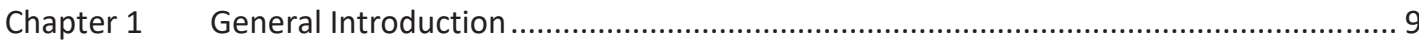

Chapter 2 Infrastructures of expertise: policy convergence and the implementation of the EU Nitrates Directive in Poland

Chapter 3 The usability of scenario studies: the case of EUruralis from the users' perspective

Chapter 4 How to create usable knowledge in a public procurement contract: the role of a knowledge broker

Chapter 5 How policymakers' demands for usable knowledge shape science-policy relations in environmental policy in Poland ...................................................................... 103

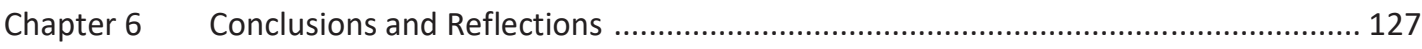

References

Summary

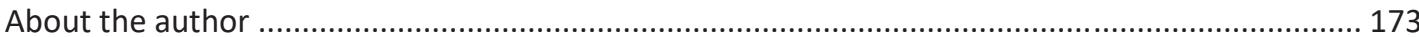


CHAPTER 1

\section{GENERAL INTRODUCTION}




\subsection{The changing role of science in policy and society}

The role of science in policy and society has been discussed by various scholars through time. An early contributor to that debate, Weinberg (1972), wrote that whereas scientists and science can provide the means for making policies, politicians and politics decide on the ends. He stated that "many of the issues that arise in the course of the interaction between science or technology and society [...] hang on the answers to questions which can be asked of science and yet which cannot be answered by science" (Weinberg 1972, p. 209). Weinberg called the answers to these questions the domain of 'trans-science' in an effort "to defend science itself from getting too implicated in areas of uncertainty" (Jasanoff 2011, p. 2). Two decades later, Beck (1992) wrote that science and policy had become collapsed in a 'risk society' due to modernization processes. A collapse which displaced the traditionally dominant role that science previously played in environmental policy. Science was traditionally considered as the only privileged form of knowledge to inform policy solutions, however the growing uncertainties and risks associated with modernity have led to science becoming politicized and openly contested in the public sphere. Accordingly, there has been erosion of trust in science and institutions.

In response to the erosion of trust in science in society, Funtowicz and Ravetz (1993, p. 86) argued that when "facts are uncertain, values in dispute, stakes high and decisions urgent", there is need for post-normal science that requires new forms of quality assurance based on an "extended peer community" and incorporating "extended facts" (Funtowicz and Ravetz 1993, p. 114, 115). In addition, Jasanoff $(1990,2011)$ argued for elaborating on the concept of regulatory science. She stated that "in regulatory science the problem of deciding what will count as adequately objective is a real issue that one has to grapple with it and think about: what is adequate for the purposes that we strive to serve" (Jasanoff 2011, p.9). She said that the point for regulatory science is not to get at the truth per se but to achieve a serviceable truth (Jasanoff 2011). These "serviceable truths" satisfy tests of scientific acceptability and support reasoned decision making (Jasanoff 1990). Hence today, there is a broad consensus that the role of science in society has been changing, where science in itself or as an isolated discipline is no longer able to serve policy and society. 
The changing role of science in policy and society has been well captured by the concepts of Mode 1 and Mode 2 of knowledge production and use, offered by Gibbons et al. (1994) and Nowotny, Scott, and Gibbons (2003). They use the term Mode 1 to denote the conventional model of knowledge production within scientific disciplines. In this model, science-policy relations are linear and "problems are set and solved in a context governed by the (largely academic) interests of a specific community" (Kraak 2000, p. 35). So, Mode 1 knowledge production is discipline-based, motivated by scientific knowledge alone (basic research) and not (per se) interested in knowledge application and innovation. The idea that basic research will automatically lead to societal benefits has been criticized for being idealistic and politically naïve (Wyborn 2015). The idea of production of science for science's sake alone, or science in isolation, is thus thought to result in less use of scientific knowledge in policy decisions. According to Gibbons et al. (1994), these problems are addressed in Mode 2, in which knowledge is produced in a context of application and can involve a much broader range of social perspectives. While peer review serves as quality control in both models, in Mode 2 it involves a more temporary and heterogeneous set of participants who are interactively involved in discussions on contextualized problems. Mode 2 is thus based on inter- or transdisciplinarity, heterogeneity of participants, and organizational diversity. As a result, Mode 2 is thought to be more socially accountable and reflexive than Mode 1 (Gibbons et al. 1994, p. 3-8, 167; Nowotny, Scott, and Gibbons 2003).

It is important to emphasize that scholars like Gibbons (1998) have not argued that Mode 2 is going to eliminate the old paradigm of Mode 1. While the shift from Mode 1 to Mode 2 was originally presented as a historical description of reality, Mode 2 has also taken on a normative meaning of a more desirable mode of knowledge production. The Mode 1/Mode 2 discussion is moreover embedded in multidirectional changes in thinking about ways of knowledge production and use: from mono to inter- and transdisciplinary approaches (Klein et al. 2001; Klein 2004; Regeer and Bunders 2009); from linear to multidirectional and social processes of knowledge co-production (Jasanoff 2004); from the sole involvement of science to the inclusion of multiple knowledge systems and knowledge holders (also captured by Funtowicz and Ravetz 1993 in their concept of post-normal science); and from an emphasis on knowledge production to an emphasis on knowledge use, application, and policy relevance (Mode 2 of Gibbons et al. 1994). 
Today, the societal trend is towards more complex and interactive models of knowledge production that include users (Metze and Turnhout 2014); Mode 2 as offered by Gibbons et al. (1994) is one of these models. Another model is found in the work of Jasanoff on the dynamic interaction between the production of knowledge and social order called co-production (Jasanoff 2004). According to Jasanoff, the term co-production includes the mutually constitutive, interactive, and influential arrangements of knowledge-making and decision-making in various aspects of political life-in other words knowledge both shapes and is shaped by social processes. She writes that "the ways in which we know and represent the world (both nature and society) are inseparable from the ways in which we choose to live in it" (Jasanoff 2004, p. 2). Therefore, the production and use of knowledge is part of social, cultural and political dynamics and cannot be separated from how we act in and organize the world.

\subsection{Normative and interventionist perspectives: moving from Mode 1 to Mode 2}

As scholars have attached a normative and interventionist interpretation to concepts such as Mode 1, Mode 2, and co-production, they are increasingly turning these concepts into instruments or methods to improve science-policy and society relations and to effectively connect knowledge systems with user demands. For example, a more instrumental interpretation of the concept of co-production has been proposed by Lemos and Morehouse (2005) and Muñoz-Erickson (2014) who see co-production as a set of collaborative processes focused on generating usable knowledge targeted at particular policies or problems. The idea is to motivate "greater interactions between producers and users of knowledge to examine the policies, processes, and capacities required to bring scientific knowledge into action" at the science-policy interface (Wyborn 2015, p. 294; also in Cash et al. 2006; Dilling and Lemos 2011; Muñoz-Erickson 2014).

The science-policy interface is another concept that is closely associated with efforts of connecting knowledge systems with user demands (Van den Hove 2007; Bradshaw and Borchers 2000; Holmes and Clark 2008). It is used to highlight the importance of managing existing science-policy interactions to ensure their effectiveness in producing usable knowledge for policy and decision makers. Greater interactions can also be a mean of reconciling supply and demand of knowledge (Sarewitz and Pielke 2007). 
The concept of transdisciplinary research has also been developed into a method or a tool with its own handbook and training methods (Bergmann et al. 2012; Hadorn et al. 2008). Rogeer and Bunders (2009) call transdisciplinarity the methodological or practical translation of Mode 2. In other words, transdisciplinarity signifies a move from analysis and descriptions of shifts (the main focus of the mode $1 /$ mode 2 argument) to intervention. Transdisciplinary research means "striving for concrete problem solving in a social and political context through cooperation between science and society" (Hadorn et al. 2008, p. 43). This term emphasizes a move beyond disciplines and advocates the inclusion of non-scientific forms of knowledge. The handbook recommends participatory processes in transdisciplinary research to carefully structure, sequence, and select negotiations and interactions (Hadorn et al. 2008). Moreover, for collaborative efforts of integration "combining different means of integration i.e. developing joint theoretical frameworks, applied models, and concrete common outputs - in an iterative or circular process - has proven to be particularly successful" (Hadorn et al. 2008, p. 437). In transdisciplinary research, there is a clear demand from science "to be conscious and explicit in terms of values and in terms of the boundaries of knowledge and findings" (Hadorn et al. 2008, p. 441).

Improving science-policy and society relations and connecting knowledge production and use are complex processes, and this complexity has several implications. First, some scholars claim that effective processes of knowledge production and use with high uptake in policymaking only have these qualities because they actually exclude actors and knowledge. Participatory processes often depoliticize the issues and fall into the trap of the information deficit model (Chivers and Kearners 2015). For example, although policymakers can be informed by a reliable, knowledgeable and hierarchical scientific community in the form of simple commands and generic instruction, increased access to information does not necessarily change their views. Second, there are always risks of colonization of one domain by the other: science may take over policy which leads to technocracy, or policy takes over science by prioritizing its own demands, which has been referred to as the merger of knowledge with power (Ravetz 1990) or simply the politicization of science (Pielke 2007). There can also be competing knowledge coalitions that use and reject knowledge based on vested interests (Turnhout, Hisschemöller, and Eijsackers 2008). Finally, certain demands for science to deliver evidence may lead to privatization of science (as sometimes is the case in specific industries 
like pharmaceuticals) which also involve risks of diminished scientific credibility and quality due to a lack of legitimate knowledge production processes.

Given the above, there is a broad consensus amongst scholars that the role of science has changed in policy processes and society. Multiple scholars moreover have argued how science may shift in multiple directions in order to make it relevant and applied in policy. Specifically, there is no consensus on the most important aspects of these shifts and what they resemble. A literature review shows that there is no one direction of shift however there are a number of intersecting developments that pull science and its connection to policy and society in different directions. Within this field, many scholars often focus on how science should be more sensitive to policy and user demands (Cash et al. 2006; Weiss 1995) whereas less focus has been placed on how policy and user demands pull science and in which directions (Bielak et al. 2008; also emphasized by Mr. Moedas, the EU Commissioner for Research in Wilsdon, Doubleday, and Stirling 2015).

\subsection{Role of the EU in steering relations between science, policy and society}

The European Union (EU) plays an important role in steering science, policy and society relations by emphasizing the importance of science and policy co-production, the involvement of users and other stakeholders in policy process, and forming policies based on high-quality science. Both co-production as well as involvement of users and other stakeholders is being promoted within the research culture in the EU, thanks to EU research programming as well as the EU culture of policy-making and policy implementation. To illustrate the role of the EU in steering science, policy and society interactions, three aspects are discussed below: 1) EU research programming, 2) EU policymaking, and 3) implementation of the EU law.

First, research together with technology and innovation are seen as the core of the European economy and vital for a successful society. Over the years, the EU “...has gradually acquired the sense of deliberate collaboration between European countries linking first their research activities, then their policies in this field" (O'Donnell and Deighton 2015, p. 3). Part of this effort is an attempt to shift knowledge production processes in order to produce relevant knowledge for policy and society and better connect knowledge systems with policy demands. What is aimed for is a change in the science-society relations of the EU, called by Stirling (2006) 
as a shift from 'science and society' to 'science in society', and finally to 'responsible research and innovation' (Felt et al. 2013). This shift is especially visible in the building of EU research programming where over the years research programmes changed in size, scope, and ambition (O'Donnell and Deighton 2015).

EU research programming started in the 1950s with the establishment of the first research programme for the coal, steel and atomic industry. Then, separate programmes for energy, environment and molecular biology were launched. Since the 1980s, a process of setting up research initiatives and putting them together in a single coherent framework started (O'Donnell and Deighton 2015). The First Framework Programme was launched in 1984 with a total budget of EUR 3.3 billion. In 1996, the Single European Act for the first time included a specific chapter on research emphasising a need for applied research to support the competitiveness of the European industry (O'Donnell and Deighton 2015). The most recent, eighth Framework Programme called Horizon 2020 has a budget of 80 billion EUR and places a great deal of emphasis on: i) engagement of participants from several countries (international approach for research became a norm in Europe); ii) widening priorities of research to include societal challenges; and iii) creating large-scale partnerships involving different sectors, industry and researchers across member states (O'Donnell and Deighton 2015). In addition, the Horizon 2020 promotes throughout its objectives responsible research and innovation approach (Felt et al. 2013; European Commission 2019).

Second, there is a certain policy culture in the EU that favours co-production and the Mode 2 type of knowledge production. This policy culture (as well as the important role of science in creating European policies) is being promoted by workshops and special expert groups acting under mandate from the European Commission (EC) (Felt et al. 2007; Stirling 2006). Specifically, this policy culture refers to the grounding European policies in expert advice, including scientific, by respecting principles of accountability, plurality and integrity when collecting and using this expert advice (European Commission's White Paper on European Governance 2001).

It also refers to using different and high-quality expert advice at every stage of the policymaking cycle and searching for mechanisms how high-quality expert advice can best inform policy, advise policy-makers, and better connect science and policy. In particular, the EC plays 
a fundamental role in promoting science and its use within EU policymaking processes and sets guidelines for what good quality knowledge is (European Commission 2002). According to these guidelines, good quality knowledge contains excellence, independency and pluralism. The same EC guidelines promote bringing scientific expertise to bear on each stage of the policy-making cycle. The need to use knowledge within the policy-making cycle is institutionalized via EU directives and regulations which demand: identification of the problem for a policy action or response (including foresight exercises), providing policy options (including impact assessment), formulating policy solutions/proposals, implementation, monitoring, review and evaluation (European Commission 2002).

The EU uses different types of expertise ranging from complex computer models and cost-benefit analyses, to simple check lists, decision trees, to ex-ante and ex-post evaluations (Nilsson et al. 2008). Expertise may also be invoked for specific national or regional situations (European Commission 2002). For example, specialized knowledge is sought by the EC both within its own Joint Research Centre (JRC) and at other organizations that can deliver professional and technical expertise such as universities, research and consulting institutes. This is because the EC has limited resources to act as a repository of knowledge and to gather and process new specialised information by itself (Sanderson 2006). But knowledge can also be gathered from non-scientific actors like citizens and non-governmental organisations. Within the policy consultation process (for which the EU has set certain minimum standards) a broad range of actors may participate including non-governmental organizations and private citizens. Proposing any new legislation (in the form of green or white papers) is always supported by an open multistage consultation process. This consultation process is primarily based on the work of formalized committees, stakeholder groups, and expert groups.

Third, EU directives and regulations institutionalize the need to collect and use expertise at both the EU level as well as at the national level. Member states require a sufficient level of expertise within their own national context in order to implement directives. In many of the EU environmental directives, for example, different implementation pathways have been applied by member states due to different national environmental conditions, different national policy cultures (an example can be the Natura 2000 network, see also McCauley 2008; Mocsari 2004; Van der Zouwen and Van den Top 2000) and also different institutional fit with the EU requirements (Borrass, Sotirov, and Winkel 2015). In many cases, the EU environmental 
directives call for a certain kind of expertise which is the use of common and standardised categories (Waterton and Wynne 1996; Waterton 2002; Turnhout et al. 2015) that member states need to deliver. These include pre-established scientific categories to designate protected areas, zones, and/or water bodies where policy intervention is required to take place (Behagel 2012). Implementation progress is reported on and monitored through these and additional scientific categories, often also developed at the national level of member states. Finally, even when specific types of expertise are not required by EU directives, these directives are nonetheless likely to act as catalysts for the production of new policy expertise at national levels (Bouwma, Arts, and Liefferink 2017).

The transposition and implementation processes of various EU directives also leads to a number of interactions between EC officials and particular member states (Héritier and Lehmkuhl 2008). In these interactions, the EC imposes its research culture, as well as its demands for scientific expertise and ways of structuring the policy processes. Member states are assisted in these interactions and provided with, for example, implementation plans, guidance documents, scientific expertise, online information, as well as support within the expert-group meetings where they can share knowledge and experiences (European Commission 2018). This support refers also to encouraging member states to use expertise in policy implementation processes according to EU standards - hence high-quality science - and thus pursue dialogue with the EC in further policy developments. The EC also has a duty to monitor member states' actions in implementing the law and ensure that their legislation and practice is compliant with those policies (European Commission 2017). If national authorities of a member state fail to comply with EU law properly, the EC may launch a formal infringement procedure against the country or even refer the case to the European Court of Justice. As shown in an article by Héritier and Lehmkuhl (2008), the European Court of Justice plays a crucial role in strengthening the actions of the EC. In this way, the EC retains its control over national policy processes and has the power to impose its own research culture on member states.

\subsection{Relations between science, policy and society in Poland}

Poland exemplifies a country in which many national processes related to formulating policies and science-policy relations are steered by EU policy processes. The pre-accession and 
accession period of Poland to the EU required many environmental directives and rural development regulations to be introduced and transposed to national Polish law in order to comply with EU rules. It also required the production of knowledge for policy convergence with the EU. Specific administrative structures and policy styles were needed (Knill 2001) in which knowledge and expertise would play an important role by providing policymakers with good quality data sets, indicators, interpretations, calculations, and new ideas to create policy solutions in particular policy sectors, including both environmental and rural development policy. The EU membership also implied that Poland needed to introduce multilevel administrative systems and ensure participation of different stakeholders in policy processes in order to comply to the EU policy culture. Hence, Poland is a good example of how the formulation of national policies and science-policy relations are steered by the EU policy processes.

Poland has struggled to comply with EU environmental policy requirements, for which multiple reasons are reported. First, Börzel and Buzogány (2010) indicate a clash of the EU culture of policy making and research with a different tradition of doing policy in Poland. This different tradition is partly a heritage of the previous socialistic system that was in Poland before 1989. The collapse of the socialistic system in 1989 resulted in transition processes to build democratic systems, based on a market economy and private ownership, and new institutional structures both in science and policy (Gorton, Lowe, and Zellei 2005). Even so, the tradition in policy remained more top-down and a shift towards a more plural, deliberative, common, interactive, and interdisciplinary focus did not (or only partly) take place. So, at the time of its EU accession in 2004, a top-down tradition of policy dominated in Poland, with a strong role of state administrative capacities in setting policy which often contradicted with EU calls for empowerment of non-state actors in the policy process (Börzel and Buzogány 2010). At that time, Polish institutions and cultures of expertise misaligned with the EU regulation's requirements for setting out terms of institutional cooperation, information infrastructure, and culture of knowledge production and use. Hallstrom (2004) observed weak capacities of administration to do environmental policymaking and an absence of domestic and supranational lobbying experience which hindered civil society groups (that would also make use of science) to actively use the opportunities provided by the EU multilevel system (also in Krammer 2004, Börzel and Buzogány 2010). 
Second, Zybała ( $\left.\begin{array}{lllll}2 & 0 & 1 & 3\end{array}\right)$ reports that a significant amount of academic knowledge is produced in Poland, both analytical and empirical, but this knowledge does not always align with the needs of policymakers. The format in which this knowledge is presented does not allow its use in policy programming (Zybała $2 \begin{array}{lllll}0 & 1 & 3\end{array}$ ). Moreover, Jelokeler, and Worek (2010) state that although growth in the body of knowledge produced is visible, they consider current datagathering systems as insufficiently developed to support public policies: data is dispersed and fragmented, not reliably evaluated, nor is it properly presented. In addition, Zybała ( $\left.\begin{array}{lllll}2 & 0 & 1 & 5\end{array}\right)$ describes a typical approach of Polish policymakers that is characterized by a lack of desire to deepen understanding about policy problems and to participate in the processes of deliberation. According to Zybała ( $\left(\begin{array}{llll}2 & 0 & 1 & 5\end{array}\right)$, this leads to a lack of institutional capability and a lack of expertise. Additionally, Zybała goes on to add there is a lack of concern for the development of modern methods of problem analysis, and a lack of willingness to engage in science-policy dialogues.

Third, inefficient science-policy communication is common in many countries and institutions. This inefficiency leads to a failure to bridge the knowledge-practice gap (e.g., Johnston and Soulsby 2006; De Koning et al. 2014). Problems may occur due to boundaries between science and policy related to differences in values, knowledge needs, requirements, and interpretations. In addition, civil servants can exhibit a tendency to believe that their experiences are unique and better than those of others. Likewise, scientists often perceive policymakers as science enemies who are not interested in scientists' results (Pielke 2007). This lack of common understanding hinders reconciliation between the supply and demand of knowledge (Sarewitz and Pielke 2007), and so hinders the production of usable knowledge. Therefore, it is not surprising that many authors claim that knowledge is often not used effectively (e.g., Meffe 1998; Bradshaw and Borchers 2000; Lawton 2007; Pohl 2008). As a solution, scholars have proposed instruments and methods to improve relations between science, policy and society (interventionist perspectives of concepts like Mode 2, co-production, and transdisciplinary research as presented in section 1.2). These instruments are used, more or less effectively, within EU institutions and member states, including Poland. 


\subsection{Research objective and research questions}

In view of the general developments in science-policy-society relations towards effectiveness and participation introduced in sections 1.1 and 1.2, the uptake of these developments in the EU, as discussed in 1.3, and the specifics of the Polish context discussed in section 1.4, the objective of the thesis is to investigate how the implementation of EU environmental and rural development policy has shaped science-policy-society relations and the use of knowledge in Poland. This objective includes both an exploration of how policy demands, formed in the case of Poland by EU requirements and a national context, affect both science-policy relations, and multiple directions of shift within these science-policy relation changes.

To reach this objective, a main research question and three sub-questions were formulated, which will be addressed in subsequent chapters.

\section{Main Research Question:}

How are science-policy relations in environmental and rural development policy in Poland shaped by EU policy processes?

\section{Sub-questions:}

A. How do European and national policy cultures and institutions shape science-policy relations? This question examines how science-policy relations are shaped by processes like Europeanization as well as institutional and cultural contexts at the national level.

B. How are science-policy interactions managed and/or steered in specific policy projects and programmes? This question examines how interactions between science and policy can be steered in specific directions (with the help of knowledge brokers and boundary objects), taking into account the trade-offs between different criteria for usable knowledge, and considering specific policy projects and programmes.

C. How do user and policy demands affect science-policy relations? This question investigates how empirical examples of policy and policymakers' demands of knowledge use, including political and instrumental use, affect science-policy relations and lead in the direction of either Mode 1 or Mode 2 knowledge production. 


\subsection{Conceptual background and analytical approach}

\subsubsection{Conceptual background}

In order to investigate how the implementation of EU environmental policy and rural development policy has shaped science-policy-society relations and the use of knowledge in Poland, this thesis adopts a constructivist approach. A constructivist approach to research aims to understand the world of human experiences as continuously shaped through human interaction with objects and other subjects (Cohen and Manion 1994, p.36). In a similar vein, Schwandt (1994) explains that "knowledge and truth are created, not discovered by mind. [...] They emphasize the pluralistic and plastic character of reality - pluralistic in the sense that reality is expressible in a variety of symbol and language systems; plastic in the sense that reality is stretched and shaped to fit purposeful acts of intentional human agents" (p. 236). Additionally, social constructivists assume that the world can be understood via social artefacts which are "[...] products of historically situated interchanges amongst people" (Gergen 1985, p. 267).

The constructivist approach allows researchers to show how things, like knowledge production processes, are shaped through social processes and values. It requires paying close attention to, and understanding of, how knowledge is constructed by individuals. It also considers knowledge as subjected to the same processes that can characterize human interactions such as communication, negotiation, and conflict (Schwandt 1994; Fischer and Gottweis 2013). It requires analysing values, considerations, and interpretations of people who create this knowledge. A constructivist approach also helps researchers understand policymaking processes by analysing activities, contributions, and communication practices of policy professionals and others involved in the policy processes and how they are engaged in struggles over power and policy (Fischer and Gottweis 2013). As knowledge is considered entwined with policymaking, this adds another dimension and expands analysis to how social practices of knowledge production and use are ordered within various institutions and whether this ordering is appropriate and/or acceptable.

Science, as socially constructed, has never been value-free and this has implications for knowledge productions processes. As a social construction, science critically investigates concepts like Mode 1 which portray science and policy as separate worlds. In science and 
technology studies (STS), therefore, scientific ideas and concepts are considered as also social constructions that are created by ideas, events, and objects within a series of choices and interactions. These interactions may change perceptions that different groups of actors have on these constructs. This has been highlighted for example in the concept of co-production in the interpretation of Jasanoff (2004), where science-policy relations are seen as coiled about each other so that it is not possible to fully separate the two. The concept of co-production in STS is used for critical assessments of science, policy and society relations (Jasanoff 2004). The co-production metaphor allows researchers to better understand how knowledge shapes perceived reality and vice versa hence what we know cannot be divorced from how we act. In other words, social processes and normative influences are inextricably intertwined (Wyborn 2015). Wyborn (2015) clearly explains that in STS the concept of coproduction helps "to examine how knowledge is framed, disseminated, and legitimized within particular social contexts and how the processes and institutions of knowledge production and culture in turn shape social order" (Wyborn 2015, p. 294).

\subsubsection{Analytical approach}

This thesis focuses on investigating science-policy relations and knowledge use in certain policy contexts. To do so, it applies analytical concepts from the field of STS as well as from governance and Europeanization studies. It focuses specifically on the following three aspects that are important for science-policy relations and knowledge uses: 1) institutional and cultural context, 2) intervention management, 3) policy demands for knowledge use.

\section{Institutional and cultural context}

Science-policy relations and knowledge production processes are not isolated but always embedded and situated. Institutions and culture impact on what is seen as relevant knowledge and what modes of knowledge production are considered to be reliable. Institutions set rules for institutional communication and interactions between state institutions and societal actors and by this mechanism create culture and regulatory style. These rules are related to modes of governance that can be hierarchical, market-oriented or multi-actor. The role and nature of legal institutions varies amongst polities and countries (Halffman 2005) and these rules also refer to practices of using expertise in regulatory 
processes. Differences between countries can also include the organization of interactions between policy and expertise (Renn 1995).

By referring to culture, this thesis concentrates mostly on policy culture. Policy culture can explain how and why actors invest time in something and give it meaning (Wedeen 2002). A focus on policy culture also enables researchers to understand beliefs, values, and customs of a specified group (Huntington 1993). Rogowski (1997, p. 14) believes in the existence of "respective cultures" with possible "cultural peculiarities" and "irreducible differences", so that "culture" refers to a "socially shared and logically interrelated set of symbols, codes, and norms". Policy cultures include styles of debate, the relative importance of legal institutions, the role of elites, and the extent of public participation (policy cultures are also discussed under the name of administrative style). These cultures can differ significantly between countries, as well as between policy sectors (Halffman 2003; 2005).

Institutions and policy culture are closely intertwined with each other and together create a context in which science and policy operate. The works by Halffman $(2003 ; 2005)$ and Jasanoff (2005) offer several examples in the regulation of toxicology and biotechnology that demonstrate the mutual constitution of policy and expert institutions and cultures. In a similar vein, studies in global climate and biodiversity governance have pointed to the ways in which knowing and governing have become entwined (Jasanoff et al. 2004; Hulme 2010; Turnhout et al. 2014; Turnhout et al. 2015).

In the field of science and policy, institutional and cultural context come together in 'infrastructures of expertise' (Kowalczewska, Behagel, and Turnhout 2017). Infrastructures of expertise are developed to specifically suit individual countries and policy sectors. For example, an institutional practice of expertise may be governed by formal and explicit boundaries between roles and responsibilities of scientists and policymakers, including strict rules of engagement (Halffman 2003; 2005). A cultural practice of expertise may consist of informal interaction between scientists and policymakers through elite networks and with blurred boundaries. These infrastructures of expertise are also known to be part of civic epistemologies (Jasanoff 2005). According to Miller (2008), civic epistemology concept "refers explicitly to the social and institutional practices by which political communities construct, review, validate, and deliberate politically relevant knowledge. Civic epistemologies include 
the styles of reasoning, modes of argumentation, standards of evidence, and norms of expertise that characterise public deliberation and political institutions" (Miller 2008, p. 1896). They can be specific for individual countries and policy domains and not easy to change.

The EU has its own civic epistemology. In the EU, both institutional and policy practices and culture are an integral part of European governance. Studies on EU accession discuss what they call the process of Europeanisation: how member states become part of a single European political space. This political space is ideally understood to be a single system of governance (Olsen 2002). However, important differences between EU civic epistemology and member state's civic epistemology can exist, different systems of governance as well as institutional and cultural traditions may dominate, or some traditions may be lacking, and the case study of Poland presented in this thesis illustrates this well. Accordingly, accession of Poland to the EU invokes a process of policy convergence as policies in Poland have to become Europeanised; and this calls for adherence to specific 'administrative structures and styles' of member states (Knill 2001, p. 2). The outcome of policy convergence is the alignment (more or less effective) of member states' institutional frameworks and policy cultures with the requirements of the civic epistemology of the EU.

\section{Intervention management}

The connection between science and policy is not self-evident. There is continuous debate about how policymakers use or do not use scientific knowledge in policymaking processes. There are also complaints about the difficulty of reaching and balancing an effective use of knowledge in policy and joint knowledge production. Subsequently, as already mentioned in section 1.2, scholars have attached a normative and interventionist interpretation to concepts describing science, policy and society relations and turned them into instruments or methods to improve the connections of knowledge systems with user demands and hence a science-policy interface. Not only do many projects emerge to manage and facilitate sciencepolicy interface but also many concepts occupy this field, including a number of variations of the "boundary" metaphor.

In the interventionist perspective, concepts of boundary work and boundary objects are seen as tools to create better interactions amongst science and policy actors. In order to connect these two domains across boundaries, the use of boundary objects (Star and Griesemer 1989) 
is relevant. Boundary objects enable intercommunication between different groups (Riesch 2010). Boundary objects are seen as an "analytic concept of those scientific objects which both inhabit several intersecting social worlds [...] and satisfy the informational requirements of each of them. [...] They have [a] different meaning in different social worlds but their structure is common enough to more than one world to make them recognizable, a means of translation" (Star and Griesemer 1989, p. 393). Therefore, boundary objects may be helpful in facilitating discussion, negotiations, facilitating communication and they can offer a common vocabulary (Carlile 2002; Turnhout 2009). Examples of boundary objects include policyrelevant tools such as scenarios, study, models, and indicators. They allow for different interpretations and meanings in different social worlds as well as meeting the demands of all involved (Star and Griesemer 1989).

The literature also provides a concept of specific individuals as 'knowledge brokers' who work either within science or policy or are located in intermediary or so-called boundary organizations (Boari and Riboldazzi 2014; Saarela et al. 2015) and can facilitate connections between different groups. These knowledge brokers draw, overcome and manage boundaries. They play a fundamental role in the process of facilitating exchange between sources of knowledge and users of knowledge which is crucial to enhancing the usability of knowledge (Bielak et al. 2008; Lomas 2007). The literature has variously specified a broad set of functions, activities and roles of knowledge brokers (Turnhout et al. 2013; Michaels 2009; Meyer 2010; Ward, House, and Hamer 2009; Oldham and McLean 1997; Bielak et al. 2008). According to Meyer (2010) and Ward, House, and Hamer (2009), knowledge brokers facilitate the transfer of knowledge by organizing connections between those who require knowledge and those who can supply it. They can also identify specific knowledge needs by creating space for the sharing of different interpretations and perceptions about expertise, knowledge and knowledge use (Van Pelt et al. 2014). They deal not only with the content of a knowledge need but also with its process (collaboration, communication, sharing, and common understanding). They can use a variety of tools such as face-to-face contacts, meetings (stationary or online), workshops, trainings, and tools such as databases, schemes, templates. Beyond creating connections, knowledge brokers may also create knowledge itself by becoming active participants in the process. (Holzmann 2013; Ward, House, and Hamer 2009; Goffin et al. 2010). 
Both boundary objects and knowledge brokers can foster collaboration between science and policy and enhance the joint and interactive production of usable knowledge. An interesting contribution for evaluating the management of science-policy interactions has been provided by Cash et al. (2002; 2003) who proposed criteria of credibility, salience and legitimacy for scientific knowledge. Credibility refers to validated and reliable knowledge, of quality related to science excellence (use of reliable methods). Salience refers to the relevance for a user, including the timing of delivering knowledge. Legitimacy refers to the fairness of knowledge production and use process, including issues of interactions, cooperation and participation. Cash et al. (2003) recognise that overlaps and trade-offs between the criteria exists and that these overlaps are ambiguous and subject to multiply potentially competing interpretations. This means that every actor involved in a debate brings their own view on what is credible, salient and legitimate. Therefore, processes of communication and interaction with the help of boundary objects or knowledge brokers provide chances to reach acceptable levels for all three criteria across actors.

\section{Policy demands for knowledge}

There is considerable discussion about the demands that users make of knowledge and what makes this knowledge usable for them. Defining criteria for usable knowledge is an important topic in knowledge-utilization studies and the literature provides references in relation to many policy sectors. Scholars have come up with partly overlapping and different sets of criteria of what is usable knowledge (Rich 1991; Weiss 1995; Dunn and Laing 2017) and have also indicated trade-offs between criteria (e.g., Cash et al. 2002; 2003). However, the debates about the criteria and what is usable often focus on the supply side of the science-policy interface. However, "the 'demand-side' is equally important: advisory bodies need a sophisticated understanding of how policymaking processes work, and the pressures and constraints under which politicians, officials and decision makers operate" (Wilsdon, Doubleday, and Stirling 2015, p. 17). Therefore, this thesis focuses specifically on policymakers' demands towards usable knowledge.

Policymakers' demands towards knowledge may place different emphasis on different criteria depending on the intention of use as well as on the general policy context in which they are situated. What criteria are considered for knowledge has been, for example, analysed by Weis 
(1995) who described five criteria important for usable knowledge: 1) relevance, 2) conformity, 3) quality, 4) action orientation, and 5) challenging the status quo. A recent study by Dunn and Laing (2017) shows, however, that users such as policymakers under certain conditions do not necessarily desire knowledge to be legitimate and credible as much as they prefer it to be policy-relevant. They therefore propose that research should consider 'applicability, comprehensiveness, timing, and accessibility' (ACTA) (Dunn and Laing 2017) rather than 'credibility, relevance, and legitimacy'. The latter set of criteria, as also described in the previous section, have been made popular by Cash et al. (2002) and have indeed been rapidly adopted by the scientific community to serve as a standard by which to evaluate knowledge production and use. Both sets of criteria can be used 'to better understand competing demands at the science-policy interface' (Tangney 2017, p. 149).

In relation to intended use, there are also several concepts that describe how policymakers may use knowledge in policy processes. Weiss (1979) presents the idea that knowledge can enlighten (knowledge is used to shape views), can be used tactically (knowledge is deployed as a tool to show that action is being taken) or politically (knowledge is used to undermine particular position). Beyer (1997) in his essay offers a synthesis of existing literature and sets three types of research use: instrumental, conceptual, and symbolic. Instrumental use involves applying research results in specific, direct ways. In conceptual use knowledge is used to generate an idea or concept. Symbolic use involves using research results to legitimate and sustain predetermined positions. (Beyer 1997).

The relation between the importance of different criteria and the intention of use in the case of policymakers is not self-evident, however, some assumptions can be made. Instrumental use of knowledge means solving particular problems like assessing the level of pollution in water or providing diagnosis of a sector (state of play). This information is crucial for deciding whether to take action through policy. Using knowledge instrumentally requires that it be relevant, delivered timely and present the truth based on facts and reliable research methods (e.g., monitoring data). The instrumental use of science in the form of numbers and numerical thresholds (on pollutant concentration in air, water, soil, foodstuffs) has some risks that Rayner (2003) describes: "Where numbers are explicitly used, the existence of competing assessment allows decision makers to select the analysis that most closely conforms to their pre-existing preference. So, while the triumph of technique promises objectivity and 
transparency, it seems just as likely to be a means to buffer decisions from public scrutiny" (Rayner 2003, p. 167).

Within politically sensitive situations, demands towards science are often expressed that call for clear independence from policymaking for example by demarcating science from policy with boundaries. In such situations, policymakers sometimes may prefer the linear sciencepolicy model like Mode 1 in the definition of Gibbons et al. (1994) to avoid responsibility and out of fear of political backlash (Valente et al. 2014). But this independence can also be obtained in a different way. Pielke (2007) shows that close interactions between science and policy do not have to interfere with maintaining scientific independence. On the contrary, interactions allow actors to mobilize specific scientific knowledge in a more open, less politicized context, that may be needed when dealing with complex problem-solving. This shows that policymakers' demands for usable knowledge could be addressed also within different science-policy relations and that depending on how the policy demands are framed, they can call for more open or closed models of science-policy relations.

\subsection{Methodological approach}

\subsubsection{Research methodology}

This study adopts a qualitative case study approach. In general, case studies are employed as a qualitative research methodology in social sciences (Yin 2017). Case study methodologies are most useful for in-depth investigations of issues in complex contexts to inform better understanding of a particular issue (Punch 2005; Flyvbjerg 2006). There are four characteristics of a case study that are relevant here (Punch 2005). First, case studies are bounded. Second, the case has to be about something specific, like a policy programme, and based on this, units of analysis are determined. Third, as written in Punch (2005, p. 145), "there is an explicit attempt to preserve the wholeness, unity and integrity of the case". Fourth, multiple sources of data and data collection methods are usually used.

The case study in this thesis focuses on Poland. Poland is an interesting country to study because its accession to the EU in 2004 not only required many laws to be introduced and/or changed in order to comply with the EU rules, like rural development policy and nitrates policy, but it also required the production of specific knowledge for policy convergence with the EU. 
This was a process in which knowledge and expertise played an important role in policy by providing policymakers with data sets, indicators, interpretations, calculations, and new ideas to create policy solutions. In this process, science-policy relations were changing, and this was also closely related to EU policymaking processes and particular demands for knowledge use.

The case study investigates how science-policy relations are shaped by EU policy processes in the context of two EU policy dossiers: 1) rural development policy, and 2) the EU Nitrates Directive, both in the period between 2004 and 2016. These two policy dossiers integrate agricultural and environmental issues and link to the European Common Agricultural Policy (CAP). The CAP was adopted in 1962 to subsidize and upscale European agricultural production (Schure and Arts 2012). It has a two-pillar structure. Whereas Pillar 1 focuses on direct payments to farmers and market management measures, Pillar 2 is dedicated to improving structural and environmental performance of agriculture and promoting rural development (Cantore et al. 2011). Environmental concerns such as environmental pollution and biodiversity loss associated with intensified agricultural practices as well as issues of climate change have gained attention over the past three decades and become integrated to both pillars of the CAP gradually since 1980, through its various instruments (Schure and Arts 2012). The rural development policy (Pillar 2) addresses issues related to reducing the degree of agricultural intensification (reducing pollution and emissions) via putting more attention to the concept of multi-functionality of agriculture, embracing spatial, environmental, service, and production functions (Amekawa et al. 2010). The EU Nitrates Directive, adopted in 1991, is one of the instruments for the protection of waters (surface, ground, and marine) against agricultural pressures, which elements are also incorporated in both pillars of the CAP (European Commission 2018; Matthews 2010).

Within these two policy dossiers, science-policy relations are investigated in the following four contexts: i) the historical implementation of the Nitrates Directive in Poland ii) Polish policymakers' demands towards the usability of a specific scenario study - the EUruralis project (Klijn et al. 2005), iii) the organization of science-policy relations in a public procurement contract focusing on mainstreaming climate change into rural development policy, and iv) Polish policymakers' demands of science and expertise in the field of rural development policy and nitrates policy. 


\subsubsection{Methods of data collection and analysis}

For the case study, multiple sources of data and multiple data collection methods were used. They included collection and analysis of data from documents and literature, interviews, questionnaires and participant observations (described in more detail in the next section) in order to ensure triangulation and internal validity of the research. Investigating science-policy relations in different policy contexts moreover strengthened the external validity of this research and allowed for the drawing of some general conclusions about science-policy relations in environmental policy in Poland.

\section{Documents and literature}

Documents are a rich source of data (Punch 2005). In conjunction with other data, documents are important for triangulation (Denzin 1989). The range of documents may include: official letters, essays, notes, institutional memoranda and reports, governmental pronouncements and proceedings (Jupp 1996) as well as statistics and records. For this thesis, the collected documents include policy documents, policy notes, governmental correspondence, scientific reports, national and EU legal statutes, project documents. While selecting documents, important criteria were: source of the documents (it had to be official and put in writing), relevance for the policy contexts, non-confidentiality, and credibility. Literature review was an additional source of data for a better historical overview of transposition and implementation of EU law in Poland and a better understanding of the policy dossiers (the Nitrates Directive and rural development policy). The table 1 below summarizes the collected and analysed policy documents, legislation, and literature used per each policy contexts. 
Table 1. Summary of used policy documents, legislation, and literature.

\begin{tabular}{|c|c|}
\hline Policy Context & Selected and analysed documents and literature \\
\hline $\begin{array}{l}\text { Implementation of the } \\
\text { Nitrates Directive }\end{array}$ & $\begin{array}{l}\text { - Ministerial correspondence and policy notes (Ministry of Agriculture } \\
\text { and Rural Development, Ministry of Environment, National and } \\
\text { regional Water Management Authorities, Chief Environmental } \\
\text { Inspectorate). These include official correspondence between } \\
\text { different institutions involved in the implementation process at the } \\
\text { national level and with the EC, letters ordering scientific reports, } \\
\text { invitations for meetings, opinions about reports, decisions, and } \\
\text { draft proposals of new national regulations. } \\
\text { - Scientific reports regarding implementation of the Nitrates } \\
\text { Directive. } \\
\text { - EU and national legislation related to the Nitrates Directive. } \\
\text { Literature on implementation of the Nitrates Directive in Poland } \\
\text { and in other EU countries. }\end{array}$ \\
\hline $\begin{array}{l}\text { Implementation of the } \\
\text { rural development } \\
\text { policy }\end{array}$ & $\begin{array}{l}\text { - Official policy document related to rural development programming } \\
\text { (both perspectives: 2007-2013 and 2014-2020). This includes ex- } \\
\text { ante evaluations, diagnosis of rural areas in Poland, rural } \\
\text { development measures, payment calculations, strategic plan for } \\
\text { rural development. } \\
\text { - Scientific analysis supporting the design and implementation of } \\
\text { particular rural development measures. } \\
\text { - EU and national legislation related to the rural development policy. } \\
\text { Literature on implementation of the rural development policy in } \\
\text { Poland and in other EU countries. }\end{array}$ \\
\hline $\begin{array}{l}\text { Usability of a specific } \\
\text { scenario study - the } \\
\text { EUruralis project }\end{array}$ & $\begin{array}{l}\text { - Technical documents of the EUruralis project: } 1.0 \text { and } 2.0 \text {. } \\
\text { - Policy correspondence of the Ministry of Agriculture and Rural } \\
\text { Development (invitations to the meeting on EUruralis, notes from } \\
\text { the meetings about EUruralis). } \\
\text { - Literature review about similar future scenarios done by Polish } \\
\text { scientists and related to Poland. }\end{array}$ \\
\hline $\begin{array}{l}\text { Public procurement } \\
\text { contract of the } \\
\text { European Commission }\end{array}$ & $\begin{array}{l}\text { - Official policy documents related to the drafting of a public } \\
\text { procurement contract. } \\
\text { - Review of official European Commission documents preparing } \\
\text { technical specifications of the public procurement contract. } \\
\text { - } \text { Reports (initial, interim and final) from public procurement } \\
\text { contract. } \\
\text { - Policy notes, minutes from the meetings between the European } \\
\text { Commission and the contract supplier. }\end{array}$ \\
\hline
\end{tabular}




\section{Interviewing and questionnaires}

Interviews were one of the main data collection tools in this research project. According to Punch (2005, p. 168) "it is a very good way of accessing people's perceptions, meanings, definitions of situations and constructions of reality. It is also one of the most powerful ways we have of understanding others". When interviews were difficult to conduct, questionnaires were used to supplement the information of the interview. In total, forty-one respondents took part in interviews and questionnaires. They represented: 1) Polish policymakers working for the Ministry of Agriculture, Ministry of Environment, National and Regional Water Management Authorities, and the Chief Inspectorate for Environmental Protection in Poland; 2) Polish scientists working for research institutes belonging to the Ministry of Agriculture; 3) Policymakers working for the Dutch Ministry of Agriculture and the European Commission; and 4) International scientists and consulting experts. The interviews were not-standardized; often respondents were asked different questions, depending on their role and expertise. The interview and questionnaire list is included in each of the chapters, without names to ensure anonymity. Interviews and questionnaires were put into written form (reports or minutes) and checked with the respondents for validity. Interviews and questionnaires were made in the period between 2006 and 2016.

\section{Participant observation}

Participant observation is an important tool for data collection. It requires changing one's role from that of a researcher to that of a detached observer of the situation, or both participant and observer of the situation (Punch 2005). In this thesis, I used participant observation during my professional working time, which includes:

- two internships (in 2005 and in 2006): 1) in the Polish Ministry of Agriculture, Rural Development Department where I learned about rural development policy and the design of rural development programmes; 2) in Alterra Wageningen University and Research, Landscape Department where I worked on the EUruralis project,

- permanent contract for the Ministry of Agriculture (since 2008 until 2014) where I was responsible for environmental aspects of the CAP (including implementation issues of the Nitrates Directive and rural development policy), 
- secondment as national expert to the European Commission, DG for Climate Action (mid-2012 until mid-2013) where I was responsible for public procurement contracts related to agriculture and climate change, amongst others.

Specific notes for data collection for this thesis were taken during nine meetings, two extensive workshops and certain phone calls. Participant observation reinforced my understanding of the two policy dossiers investigated in this thesis. In addition, I had the opportunity to observe how science and policy interacted in practice in Poland. Finally, working on secondment to the European Commission enabled me to learn how to organize a successful science-policy interface.

\section{Data analysis}

In this thesis, a qualitative data analysis has been conducted. Transcripts from interviews, participant observations, notes from policy documents and literature studies were analysed in the context of two EU policy dossiers in four different contexts, as already mentioned: 1) the Nitrates Directive's implementation in Poland, 2) Polish policymakers' demands for usability of scenario study called EUruralis, 3 ) the organization of science-policy relations in a public procurement contract, and finally 4) Polish policymakers' demands for knowledge in rural development and nitrates policy. Qualitative coding was used to analyse this written form of data. "Coding is the concrete activity of labelling data, which starts the analysis, and also goes on at different levels throughout the analysis" (Punch 2005, p. 199). Coding is seen as a process of "classifying and categorizing text data segments into a set of codes (concept), categories (constructs) and relationships" (Bhattacherjee 2012, p. 113). For each of the context situations, similar steps in the analysis were applied: 1) developing codes, 2) identifying phrases and arguments in the collected data that corresponded to the codes, and 3) ordering and summarizing the data as well as drawing conclusions. The data was coded in rounds. The first round focused more on themes that empirically come out of the collected data. The second round focused more on finding fragments of texts that link up with the analytical concepts. The coding process was supported by QDA Miner Lite software and my own tables created in Microsoft Excel.

In the context of the Nitrates Directive, analysis of the data was based on historical analysis that was produced by coding the data for the occurrence of changes in the institutional 
framework and policy culture related to scientific knowledge and expertise, building on the notions of civic epistemology (Miller 2008; Jasanoff 2005) and cultures of expertise.

In the context of a scenario study such as EUruralis, data analysis focused on: 1) the assessment of EUruralis' usability in terms of credibility, salience and legitimacy criteria, according to Cash et al. $(2002 ; 2003)$ used as codes, and 2$)$ the characteristics of the sciencepolicy relation that took place within the EUruralis project. In particular, I searched the data for how respondents described processes of cooperation and interaction and how EUruralis influenced their practices.

In the context of organization of science-policy relations in a public procurement contract, I developed codes based on a literature review of knowledge brokering activities (Turnhout et al. 2013; Michaels 2009; Meyer 2010; Ward, House, and Hamer 2009; Oldham and McLean 1997; Bielak et al. 2008) that are relevant for the interactions between knowledge production and use in the case of public procurement contracts. I proposed two categories of knowledge brokering activities such as 'translation' and 'flexible intermediary' used as codes and I looked for activities within these two categories that had been performed by a knowledge broker in a public procurement contract.

In the context of policymakers' demands for knowledge in the rural development and nitrates policy, I searched the data for policy discourses that were supporting Mode 1 or Mode 2 science-policy relations according to Gibbons et al. (1994) definition, and to identify preferences for one or the other within the two policy contexts in which interviewees were situated. In addition, I coded the data for the framing of policy demands according to the criteria of knowledge presented by Weis (1995), including whether demands for use could be identified as political or instrumental.

\subsection{Organization of the thesis}

This thesis is organized into a total of six chapters. Chapters 2 to 5 are on empirical studies and relate to particular sub-questions presented in this chapter. Each of these has been developed as an independent research paper for a peer-reviewed journal.

Chapter 2 addresses research sub-question A. It shows that the accession of Poland to the EU in general leads to a process of policy convergence in which member states' institutions and 
policy cultures become increasingly adapted to align with an EU governance system. Especially in EU environmental policy, knowledge and expertise are key aspects of the institutions and policy cultures that are adapted in this process, which ideally results in the alignment of EU policy and administrative arrangements of member states. Chapter 2 offers a historical analysis of the Nitrates Directive's implementation in Poland and shows how increasing convergence of Polish institutions and cultures of expertise with EU policy occurred in response to the directive's requirements.

Chapter 3 addresses sub-question B. It shows how scenario studies can be seen as useful tools to support planning and decision-making processes and how they play an important role in facilitating cooperation and interaction at the science policy interface. It uses a theoretical framework that connects the criteria of credibility, salience and legitimacy to the concepts of coproduction and boundary objects in order to analyse the EUruralis scenario study that addresses the future of agriculture and rural development in Europe.

Chapter 4 also addresses sub-question B. It discusses how the relation between science and policy is organized in a public procurement contract. Experiences from existing science-policy interfaces call for mechanisms for communication and exchange between scientists and policy-makers that go beyond the 'old' linear supply and demand model. This chapter shows that the public procurement contract offers good possibilities for reconciling knowledge supply with demand via knowledge brokering activities related to problem formulation, the identification of research needs, and management of the interactions between the procurer and the researcher.

Chapter 5 address sub-question C. It discusses how aspects related to policymakers' demands for knowledge shape preferences for science-policy models such as Mode 1 and Mode 2 (Gibbons et al. 1994). The chapter focuses on the demands that Polish policymakers make of science and how they envision their role in the knowledge production process in the field of environmental and rural development policy. In this chapter, a set of criteria on how policymakers define usable knowledge is applied (Weiss 1995; Cash et al. 2002; 2003) to better understand preferences and uses-in-practice of different science-policy models.

Chapter 6 presents the conclusions by answering sub-questions $A, B$, and $C$ as well as the main research question. The chapter concludes by revisiting the changing role of science and its 
attendant shift from Mode 1 to Mode 2, as well as the concept of Europeanization. It includes also reflections on methodological underpinnings and offers some broader end-reflection on the science-policy relations in the context of Poland. 
CHAPTER 2

\section{INFRASTRUCTURES OF EXPERTISE: POLICY CONVERGENCE AND THE IMPLEMENTATION OF THE EU NITRATES DIRECTIVE IN POLAND}

This chapter has been published as:

Kowalczewska, K., Behagel, J., and Turnhout, E. (2017). Infrastructures of expertise: policy convergence and the implementation of the EU Nitrates Directive in Poland. Journal of Environmental Planning and Management, 1-19. 


\section{ABSTRACT}

Access to the EU leads to a process of policy convergence in which member states' institutions and policy cultures become increasingly adapted to align with an EU governance system. Especially in EU environmental policy, knowledge and expertise are key aspects of the institutions and policy cultures that are adapted in this process, which ideally results in the alignment of EU policy and administrative arrangements of member states. This chapter offers a historical analysis of the Nitrates Directive's implementation in Poland and shows how increasing convergence of Polish institutions and cultures of expertise with EU policy occurred in response to the directive's requirements. The results highlight that 1) knowledge and expertise are central to policy convergence processes and that 2) institutions and cultures of expertise are entwined in 'infrastructures of expertise'. The chapter concludes with a call for more consideration of the science-policy interface in policy convergence processes related to Europeanisation.

Keywords: science-policy interface; policy convergence; Nitrates Directive; Europeanisation 


\subsection{Europeanisation and the Nitrates Directive}

Central and Eastern European (CEE) countries that joined the European Union (EU) in 2004 were confronted with the requirement to adopt the whole body of European law and practice known as the 'acquis communautaire' (Gorton, Lowe, and Zellei 2005). The Nitrates Directive (European Commission 1991) - protecting water against pollution caused by nitrates from agricultural sources - is an important part of this acquis communautaire. Poland, as the largest member state that accessed the EU in 2004, has already had multiple issues with the implementation of this directive. For example, Poland has been taken to the EU Court of Justice by the European Commission (EC) in 2013 as 'too little had been done' to implement the Nitrates Directive (European Commission 2013) according to the EC. In practice, many EU countries struggle with the implementation of EU environmental directives and both 'old' and 'new' member states have had infringement procedures being opened on various aspects of the Nitrates Directive (European Commission 2013). Even so, CEE countries face additional challenges to comply with EU directives as they are associated with a historical legacy of strongly centralized and state-centred forms of governance (Kluvánková-Oravská et al. 2009).

Given the above, Poland is a good example of how accession to the EU is accompanied by struggles over complying with EU requirements and processes of policy convergence (Knill 2001). What 'doing enough' means and how a member state ends up 'doing too little' is by no means straightforward: it requires insight into what is required of member states when they access the EU and how subsequent processes of policy convergence may play out over time (Leventon 2015). This is further compounded by the fact that EU governance is based on the principle of subsidiarity, which means that EU directives leave considerable scope for member states to interpret the directive's requirements and tailor their implementation to local circumstances.

Studies on EU accession discuss what they call the process of Europeanisation: how new member states become part of a single European political space. This political space is ideally understood to be a single system of governance (Olsen 2002). However, important differences between EU policy requirements and national systems of governance of new member states are present. Accordingly, accession to the EU invokes a process of policy convergence as policies of member states become Europeanized and hence call for specific 'administrative 
structures and styles' (Knill 2001, p. 2). The outcome of policy convergence is the alignment of institutional frameworks and policy cultures of member states with the requirements of the EU.

Policy convergence is often explained by referring to the extent of 'fit' between a member state and EU requirements (Frederiksen et al. 2017). According to this 'goodness of fit' theory, policy convergence will be easier for member states whose institutions and policy cultures fit well with those of the EU, while others will struggle more and may end up achieving lower levels of convergence. In this chapter, we focus on an important yet under-researched factor that influences processes of convergence: scientific expertise. Selected studies address the role of scientific expertise in terms of science-policy interfaces on the EU level (e.g., Turnhout et al. 2015), the national level (e.g., Holmes and Clark 2008), and for specific EU environmental domains (e.g., Gilek et al. 2015). However, the role of scientific knowledge and expertise in processes of policy convergence has thus far received little attention (see Radaelli 1999). This is problematic as standardised scientific knowledge and information play an important role in 'building the European Union' (Waterton and Wynne 1996). Especially in the field of environmental policy, scientific expertise is integrally linked to institutional frameworks and policy cultures (Demmke and Deakin 2001). Infrastructures of expertise are thus an important part of the institutional arrangements and policy culture that need to become aligned with EU requirements as part of policy convergence.

In this chapter, we contribute to the understanding of EU governance, and specifically policy convergence, by analysing how institutional arrangements and policy cultures related to scientific knowledge and expertise are developed in response to the implementation of the EU Nitrates Directive in Poland. We do so by showing how processes of policy convergence during the implementation of the Nitrates Directive can be seen to respond both to the requirements set by the Directive and to active interventions by the EC. Moreover, the chapter discusses the central role of scientific knowledge and expertise in these processes of policy convergence. Accordingly, our results offer an empirical and historical example of policy convergence and allow us to address a broad range of issues related to Europeanisation. Before presenting our findings, the next sections discuss the role of scientific knowledge and expertise in policy convergence and explain our case study and approach. 


\subsection{Institutions, culture, and expertise}

Policy convergence points to the process in which member state institutions and cultures come to align with EU policy requirements, e.g., by creating new governmental agencies or by demanding horizontal types of interaction between policy sectors (Knill 2001). The goodness of fit theory (Duina 1997; Knill and Lenschow 1998; 2000) states that smooth processes of convergence resulting in close alignment follow from a good fit between national administrative arrangements and those implied in European legislation (Knill and Lenschow 1998, p. 602; Liefferink, Wiering, and Uitenboogaart 2011, p. 713). Today, most EU directives combine goal orientation with a focus on specific programs on measures to allow flexibility in policy implementation on the national level (representing a 'third wave' of Directives, see also Aubin and Varone 2004). Regardless of this freedom of implementation, most EU directives still require adaptation of national institutional arrangements and policy cultures in one way or another to satisfy broader framework goals and administrative requirements such as the publication of action programs and monitoring and reporting programs.

Literature states that when there is a misfit between EU policy requirements and national institutional arrangements, the need for adaptation is high and the implementation effectiveness is likely to be low (Liefferink, Wiering, and Uitenboogaart 2011). An example of such a misfit is when EU policy requirements call for the establishment of decentralised agencies while national institutions tend toward centralisation. Policy cultures are also important factors to explain success or failure of policy convergence (see Bulmer 2008; Frederiksen et al. 2017; Haverland 2000; Knill and Lehmkuhl 1999). Policy cultures include styles of debate, the relative importance of legal institutions, the role of elites, and the extent of public participation (policy cultures are also discussed under the name of administrative style). These cultures can differ significantly between countries, as well as between policy sectors (Halffman 2003; 2005).

We argue that expertise is an important, but under-researched, dimension of European governance that is an integral part of how both institutional frameworks and policy cultures function. The implementation of EU policies entails building not just regulatory institutions but also infrastructures of expertise (Radaelli 1999) - known as science-policy interfaces - to fulfil EU requirements for the implementation of directives, including the standardisation of 
natural and geographical categories to serve as a basis for action programs (Waterton and Wynne 1996), and the measuring and reporting on implementation progress. This is a matter of adapting research and policy institutions as well as developing a new culture of expertise. In conjunction with cultures or styles of regulation, cultures of expertise are developed to specifically suit individual countries and policy sectors. These cultures of expertise are also known as civic epistemologies (Jasanoff 2005). According to Miller (2008, p. 1896), civic epistemologies refer to "the social and institutional practices by which political communities construct, review, validate, and deliberate politically relevant knowledge. Civic epistemologies include the styles of reasoning, modes of argumentation, standards of evidence, and norms of expertise that characterise public deliberation and political institutions".

The institutional and cultural practices of science and expertise refer to the ways in which experts and policymakers interact. For example, an institutional practice of expertise may be governed by formal and explicit boundaries between roles and responsibilities of scientists and policymakers, including strict rules of engagement (Halffman 2003; 2005). A cultural practice of expertise may consist of informal interaction between scientists and policymakers through elite networks and with blurred boundaries between science and policy. The works by Halffman $(2003 ; 2005)$ and Jasanoff $(2005)$ offer several examples in the regulation of toxicology and biotechnology that demonstrate the mutual constitution of policy and expert institutions and cultures. In a similar vein, studies in global climate and biodiversity governance have pointed to the ways in which knowing and governing have become entwined (Jasanoff and Long Martello 2004; Hulme 2010; Turnhout, Neves, and De Lujster 2014; Turnhout et al. 2015). As such, institutions and cultures of expertise are thought to come together in processes of policy convergence.

Within EU environmental governance, policy convergence is strongly shaped by expertise. Policy convergence by means of expertise in the EU takes place through the use of common categories and standardised monitoring and reporting frameworks (Waterton and Wynne 1996; Waterton 2002; Turnhout et al. 2015). EU environmental directives, in particular, make use of pre-established scientific categories to designate protected areas, zones, and/or water bodies where policy interventions are required to take place (Behagel 2012). Moreover, implementation progress is reported on and monitored through these and additional scientific categories, for example the category of nitrates $(N)$ concentration. Finally, even when specific 
types of expertise are not required by EU directives, these directives are nonetheless likely to act as catalysts for the production of new policy expertise (Bouwma, Arts, and Liefferink 2017).

\subsection{Case description and methods}

The implementation of the EU Nitrates Directive in Poland is taken as a case study. Adopted in 1991, the Nitrates Directive is one of the key pieces of EU legislation that aims to control pollution and improve water quality. It has close links with other EU policies concerning water, air, climate change, and agriculture. Although nitrogen is an important nutrient used in agriculture that helps plants and crops to grow, high concentrations are harmful to nature and waters (European Commission brochure 2010). Agriculture and livestock (especially manure) are responsible for over $50 \%$ of the total nitrogen discharged into surface waters (European Commission website 2016). This contributes to eutrophication, a key threat preventing good ecological status for EU surface waters under the EU Water Framework Directive (European Commission brochure 2010). Therefore, the Nitrates Directive is one of the key instruments in the protection of waters (surface, ground, and marine) against agricultural pressures (European Commission website 2016).

The Nitrates Directive sets out the following implementation requirements:

- Identification of waters that are polluted, or at risk of pollution.

- Designation of nitrate vulnerable zones (NVZs) ${ }^{1}$.

- Establishment of action programmes to be followed by farmers within NVZs on a compulsory basis, implementing good agricultural practices for farmers.

- Analyses of waters' nitrate concentration levels and trophic state. Good monitoring is crucial, and means setting up high-quality monitoring networks for ground, surface and marine waters.

- Reporting every four years to the EC on (i) $\mathrm{N}$ concentration/leaching and eutrophication, (ii) revision of NVZs and action programmes, and (iii) future trends on water quality (surface and ground) and $\mathrm{N}$ concentration. The member states' reports

\footnotetext{
${ }^{1}$ NVZs: Nitrates Vulnerable Zones - areas of agricultural land which drain into polluted waters or waters at risk of pollution and which contribute to nitrate pollution.
} 
are used as the basis for reporting on the implementation of the Directive across the EU.

The EC monitors the implementation of the Nitrates Directive. It may open an infringement procedure and refer a member state to the EU Court of Justice if it fails to guarantee that water pollution by nitrates is addressed effectively. In addition, the EC commissions external studies on different aspects of the Nitrates Directive to assist the implementation process and to expand scientific knowledge on best farming practices for the minimisation of nitrogen losses and the protection of water quality (European Commission website 2016).

Our collected data span the time period 2004-2016, a period which entailed a number of milestones for how institutional arrangements and policy cultures related to scientific knowledge and expertise developed in response to the implementation of the EU Nitrates Directive in Poland. We adopted a qualitative approach to data collection. In order to grasp a complete picture of the implementation process, our data consist of documents (ministerial correspondence, policy notes, scientific reports, national regulations), transcribed interviews, participant observation (in section 2.7.), and academic literature review.

Our data include documents that relate to the implementation of the Nitrates Directive in Poland. These include official correspondence between different institutions involved in the implementation process at the national level and with the EC, letters ordering scientific reports, and scientific reports themselves. In total, we have reviewed and analysed over a hundred pages of ministerial correspondence ${ }^{2}$. We also analysed many national regulations related to the Nitrates Directive implementation that set rules and competences for different institutions.

Interviewees were selected based on the prominence of their participation in the implementation of the Nitrates Directive and their roles in their institutions. This prominence was assessed by indication of participation in: (i) the designation process, (ii) the establishing of action programmes, (iii) meetings related to implementation (joint science-policy meetings,

\footnotetext{
2 Including letters of different Ministries presenting their positions towards the Directive implementation, discussions between Ministries on the implementation strategy, correspondence on preparing implementation reports for the EC, invitations to meetings, and letters with comments to scientific reports.
} 
working groups), and (iv) the preparation of policy and scientific reports. Eleven interviews were conducted with fifteen respondents. During the interviews, interviewees were asked to give their own historical overview of the implementation of the Nitrates Directive, including challenges that appeared, the role of science, experience of working with scientists (on what level and with what data), and the use of scientific analysis in the implementation process. Interviewees were also asked to discuss scientific analyses that were produced for the purposes of the directive. Furthermore, the first author engaged in participant observation by attending two meetings, in 2011 and in 2014, in the Ministry of Agriculture in Poland. Finally, we reviewed other scientific articles reporting case studies on the Nitrates Directive implementation in the EU to compare with our results.

The analysis of our data yields an historical analysis that was produced by coding our data for the occurrence of changes in the institutional framework and the policy culture relating to scientific knowledge and expertise, building on the notions of civic epistemology and cultures of expertise discussed above. From the moment of EU accession until now, three periods (based on EU reporting requirements) can be distinguished which we use as a chronological structure to present milestones of policy convergence during the implementation of the Nitrates Directive. These periods are as follows:

- period I: 2004-2008 (described in Sections 4.1, 4.2, and 4.3);

- period II: 2008-2012 (described in Sections 4.4 and 4.5);

- period III: 2012-2016 (described in Sections 4.6 and 4.7).

\subsection{The implementation process: $2004-2016$}

In Poland, the transposition of the Nitrates Directive requirements into national legislation resulted in several struggles over adaptation of national institutions and policy culture. These included the adoption of legal acts that regulated and listed tasks and responsibilities of various policy institutions at central and regional levels. Institutions related to knowledge and expertise equally required adapting. Policy convergence processes in areas related to cultures of expertise were also a source of struggle. The directive called for integrating environmental issues in farming practices via the designation of NVZs and the setting of rules for farmers, for which a more inter-sectoral and coordinated policy approach was needed. The reporting obligation under the Nitrates Directive also required periodic revisions of the designated NVZs and action 
programmers based on the $\mathrm{N}$ concentration state of play, again evoking processes of policy convergence in which institutions and policy cultures were adapted. We detail these processes below.

\subsubsection{Accession to the EU and the first NVZs designation}

The moment of Poland's accession to the EU has already caused a first, major challenge to its institutional arrangement and culture of expertise. Due to expected high financial costs mainly related to the construction of liquid manure tanks, Poland's initial negotiating position, as expressed by the Ministry of Environment that was responsible for this dossier, was to request an 8-year transitional period for the implementation of the Nitrates Directive (Gorton, Lowe, and Zellei 2005). The EC encouraged Poland to reconsider this position and tried to persuade Poland to prepare an implementation programme based on identified nitrates vulnerable zones (NVZs) with an investment plan to be achieved within four years after accession. The Ministry of Agriculture was expected to define the extent of the nitrate contamination problem. However, with a strong orientation on productivity, at the time of accession, the Ministry of Agriculture assigned little priority to limiting fertiliser use, and collaboration between the two ministries remained very limited (Gorton, Lowe, and Zellei 2005; Karaczun 2005; R2; R3; R6; R7). Therefore, the Ministry of Environment assigned its subordinated Institute of Meteorology and Water Management to prepare a report on the nitrate contamination problem in order to designate NVZs (R4; R5). This report stated - based on monitoring results from the period 1990 to 1999 - that there was no serious problem of nitrates pollution, that municipal sewage was the main source of high nitrates levels in surface water, and that the current levels of agricultural activity did not justify the designation of NVZs (Gorton, Lowe, and Zellei 2005; R4; R5).

Building on the expertise from the Institute of Meteorology and Water Management, the Polish Government considered nitrates contamination to be a local problem, to be solved by local authorities in the regions and by farmers themselves (Gorton, Lowe, and Zellei 2005), and no longer asked for a transitional period. The EC accepted Poland's revised position, but it also expected the Nitrates Directive to have a positive impact on minimising the nitrogen concentration in waters. Therefore, it insisted on the designation of NVZs where there was an identified problem with nitrates contamination. Acceptance of both sides (Poland and the EC) 
upon this issue closed the negotiations over the directive (Gorton, Lowe, and Zellei 2005). And so the Ministry of Environment started to prepare for the NVZs designation for the first time. Institutional competencies were adapted via legal acts by the Ministry of Environment so that regional authorities could designate NVZs in their regions and prepare relevant action programmes (R2; R3; R6; R7; R11).

Regional authorities, represented at that time by seven regional water management authorities supervised by the Minister of Environment, were neither ready for, nor knew how to deal with, the tasks they were assigned (R2; R3). The national implementation guidelines provided by the Ministry of Environment were not clear about the use of data for the designation of NVZs (R2; R3; R6; R7). The characterisation of a diffuse pollution, such as nitrate pollution, depended on monitoring data, but it was difficult to specify exactly what monitoring is required, where there is a problem, how to carry out data assessment, or how to set NVZs boundaries (Gorton, Lowe, and Zellei 2005; R2; R3). So, a lot of freedom for interpretation had been left to regional water management authorities (R9). They consulted individually with other institutions, mainly in their regions, to look for relevant monitoring data on surface waters, ground waters, and agriculture (R2; R3; R6; R7).

Regional authorities reported problems with data availability at the level of the regions (R2; R3; R4; R5; R6; R7). In addition, gathering data was difficult as datasets were fragmented and in the possession of different institutions (R2; R3). Formal rules on data sharing did not exist and institutions were reluctant to share data voluntarily (R4; R5). Regional water management authorities did not ask for data from central authorities; due to the hierarchical governmental culture this was not a practice (R2; R3). In the end, regional authorities individually commissioned analyses (based on legal contracts) in order to identify NVZs that were carried out by different scientific teams, ranging from national research institutes to private consulting companies (R2; R3; R6; R7; R11).

As research institutions were supervised by particular ministries, expertise was divided between the sectors of environment and agriculture, and communication was difficult. The Ministry of Environment supervised institutes related more to environmental sciences, such as the Institute of Meteorology and Water Management, the Institute of Environmental Protection, and the Chief Inspectorate for Environmental Protection (which set the national 
environmental monitoring network). They all provided research and data to the Ministry of Environment. Agricultural research institutes (more than ten) were supervised and partly budgeted by the Ministry of Agriculture and they provided research and data to the Ministry of Agriculture based on agreed research programmes. Not much cross-sectoral communication and coordination between scientific institutions supervised by the different Ministries was reported at the time (R1; R2; R3; R10; R11). In addition, it was difficult for the regional water authorities to contact researchers from institutes under the supervision of the ministries directly, without going through formal channels (R2; R3).

Findings from this period confirmed that the institutional organisation of expertise in 2004 did not fit with the requirements that the Nitrates Directive set out. It did not include formal ways of sharing data between policy institutions across governmental levels. A hierarchical culture strengthened this sectoral approach: communication between different levels of government (national to regional) was unidirectional, so a national body could assign tasks to a local body related to implementation, but vice versa was uncommon. The culture of expertise also did not align with requirements for data sharing and research, as well as for cooperation between regional and national authorities. Rather, expertise was organised following clear boundaries between policy and research institutions. Research outputs were requested by policymakers in formal ways and used instrumentally to solve the problem: first to agree on the accession position between Poland and the EC and then to designate NVZs, where needed, by regional policy authorities. Expertise was thus used instrumentally, where science would offer objective data to inform decisions. As will be shown more clearly in later stages of the implementation, this instrumental use of expertise sits uneasily with the idea of periodic adaptation of NVZs and action programmes.

\subsubsection{Adapting institutions of expertise}

Adapting the Polish institutional setup for the Nitrates Directive extended beyond designating NVZs. For example, the Chief Inspectorate for Environmental Protection, supervised by the Ministry of Environment, had to adapt the National Monitoring System related to waters to the requirements of the Nitrates and Water Framework Directives (R4; R5). The National Station for Chemistry and Agriculture, supervised by the Ministry of Agriculture, also received new competences on carrying out analyses of mineral nitrogen and nitrate nitrogen in waters 
in underground layers of 30,60 , and $90 \mathrm{~cm}$, which is important for the monitoring of surface water quality and mineral nitrogen values in NVZs (R10; ministerial correspondence).

In 2006, transposition and implementation of the Nitrates Directive led to the establishment of a new institution called the National Water Management Authority (the NWMA) ${ }^{3}$. The task for the NWMA was to coordinate and steer water policy, and all the associated competences had moved from the Ministry of Environment to this new institution (R11). The NWMA as a central body coordinated and also supervised regional water management authorities, including tasks related to implementation of the Nitrates Directive ${ }^{4}$. There was a lot of critique, including from the side of scientific experts, that this new institution lacked the human capacity and the competences to coordinate inter - institutional communication and shape water policy (R1; R2; R3; R4; R5; R9).

Results from this period confirm that the implementation of the Nitrates Directive called for adapting and building institutions that are closely related to expertise. Critique of the establishment of the NWMA was focused on how the development of multilevel and intersectoral institutional communication was rather difficult in a hierarchical institutional culture of communication. Our analysis indicates that although new institutions were created, old cultures of expertise perpetuated a degree of misfit in Poland's implementation processes with the requirements of inter-sectoral cooperation that the Nitrates Directive called for.

\subsubsection{The EC intervenes on the first NVZs designation}

In 2007, one year before the first implementation report was due, the EC started to investigate Poland's implementation of the Nitrates Directive (source: ministerial correspondence). DG Environment commissioned an external scientific expertise to Wageningen University and Research (WUR) to assess the designation of NVZs in Poland (R8; R12; R13; R14). The results of this expertise provided various arguments, some suggesting that the whole territory of Poland should be designated under one NVZ and one action programme, which meant a complete change of the implementation approach (WUR 2007). The Polish Government, especially the Ministry of Agriculture, did not like this, because such a change would, in their

\footnotetext{
${ }^{3}$ According to the Polish National Water Act as of 18 July 2001.

${ }^{4}$ According to the Polish National Water Act as of 18 July 2001.
} 
view, seriously affect all farmers in Poland (R1; R10). In addition, there were serious doubts about the completeness of the dataset used in the WUR analysis and about the research not taking into account positive effects of already implemented measures on manure management in Poland (R10).

Given the above, the Ministry of Agriculture decided to get actively involved in debates on the Nitrates Directive's implementation. To provide arguments for supporting the initial implementation approach (i.e. multiple, distinct NVZs), it requested the expertise of one of its research institutes - the Institute of Soil Science and Plant Cultivation in Puławy (IUNG) (R10). The summarising chapter of the resulting expert report was devoted specifically to discuss points of agreement and disagreement with the expertise of Wageningen University (Expertise of IUNG: Igras et al. 2008). In order to shape the scientific arguments, policy officers from the Ministry of Agriculture interacted intensively with scientists of IUNG during the preparation of the analysis, which resulted in mutual learning of policy and scientific actors (R10). As a result, the first implementation report that verified the designation of NVZs in Poland was based on both monitoring data and the expertise of IUNG, amongst others (R10; R11).

The institutional change that was initiated in 2006 with the establishment of the NWMA was now translated to a social practice of data sharing. The NWMA coordinated the preparation of the implementation report and collected input from regional water management authorities (R11). Although formal rules of data sharing were not officially set, now policy officers from regions knew whom to contact for data and each of the institutions was providing data in a more open manner, while regional authorities also cooperated with each other (R2; R3). The expertise of IUNG and WUR was also forwarded by the NWMA to regional authorities, so transfer of research results between policy institutions improved (R2; R3). Even so, the results of these two research reports of IUNG and WUR were published too late to be used to change the NVZs designation (R2; R3). As a consequence, the designated areas for NVZs were slightly modified (total area was reduced) and for each NVZ a separate action programme was formulated $^{5}$

\footnotetext{
${ }^{5}$ Based on Polish regulations on establishing action programmes for NVZs.
} 
The above episode shows that the intervention of the EC had an impact on the implementation process and led to the involvement of the Ministry of Agriculture. The critical evaluation of the Polish implementation approach by the EC made the Nitrates Directive become the focus of broader, political attention in Poland. Therefore, uses of science by policymakers other than instrumental appeared, such as symbolic and political uses to legitimate and sustain a preferred position. In addition, experts and policymakers interacted in a more dynamic way to build arguments that included more open data sharing. As such, the first implementation period (2004-2008) resulted in limited alignment with the EU Nitrates Directive requirements as it included the following: (i) a shift towards more coordination between institutions on sharing data; (ii) more open communication within the culture of expertise; and (iii) more types of uses of science.

\subsubsection{Further changes in institutions and cultures of expertise}

In early 2009, the NWMA officially requested the Ministry of Agriculture to be more intensively involved in the upcoming process of revision of the designated NVZs and their action programmes (source: ministerial correspondence). This request initiated a number of actions taken by the Ministry of Agriculture. First, it commissioned an analysis to a group of scientists from IUNG (based on an individual legal contract) in order to (i) assess the use of natural and mineral fertilisers in agricultural production and its pressure on the environment; (ii) assess the size of discharge of nutrients (nitrate and phosphorus) from agricultural sources based on the newest data; and (iii) project fertiliser usage and nutrient discharges for 2013 and 2015 (R10). Second, the Ministry collected other analyses related to nitrates carried out by agricultural research institutes. Third, due to an official visit of DG Environment to Poland related to the implementation of the Nitrates Directive (December 2009), the Ministry of Agriculture arranged a pre-meeting with Polish government institutions to agree on a common position with regard to the question of whether the NVZs designation approach should be changed in the future. Almost all of the institutions involved in the implementation process were invited, including the NWMA, the Ministry of Environment, the National Station for Chemistry and Agriculture, the Agricultural Paying Agency, and representatives of scientific institutes subordinate to the Ministry of Agriculture (source: ministerial correspondence). 
The stronger engagement of the Ministry of Agriculture in the implementation process and a more integrated approach at the institutional level was accompanied by a stronger recognition of policymakers of the need for support by science. This recognition was strengthened further after the official meeting with the DG Environment, during which the EC expressed its expectation of Poland to be open to further enlargement of NVZs due to eutrophication of the Baltic Sea (based on the ministerial documents). In addition, the EC underlined in its official report to the Council and the European Parliament at the beginning of 2010 that it will continue to work with member states to improve implementation with the common aim to protect waters. It will continue to take legal action where it considers necessary (European Commission 2010). This statement could be seen as a warning that the EC would take legal action if changes regarding the designated NVZs did not follow.

In early 2010, the next action of the Ministry of Agriculture focused on incorporating monitoring of the impacts of agriculture on waters' pollution and the Baltic Sea in the multiannual research programme of IUNG for 2011-2015 (financed by the Ministry of Agriculture) (R10). This followed the idea that values of nitrates contamination coming from agricultural sources should be monitored continually (R10). Monitoring was to be executed in cooperation with the National Station for Chemistry and Agriculture, the NWMA, regional authorities, and Ministries (R10). Furthermore, in mid-2010 the NWMA commissioned the University of Warsaw to prepare a model to designate river catchments with increased outflows of nitrates (University of Warsaw 2011). The Ministry of Agriculture, as well as scientists from IUNG, were invited to a final meeting during which this model was presented and discussed (source: ministerial correspondence).

This episode shows an increased institutional cooperation between government agencies to obtain datasets, scientific analyses, and identify pressures of agriculture on water quality, which could be used to better align the Polish 'infrastructure of expertise' with the requirement of NVZs and action programmes revisions. However, certain institutions still worked independently from one another: the Ministry of Agriculture and the NWMA contracted different scientific teams. Accordingly, the relation between scientific and policy organisations still remained predominantly vertical: science produces arguments that policymakers can use. 


\subsubsection{Infringement procedure against Poland and next NVZs' designation}

In October 2010, the EC sent Poland a notice with a call to address implementation failures of NVZs designation and action programmes for farmers. Poland officially replied to this notice in December 2010 and assured the EC that the action programmes and national Polish law together are sufficient to fulfil the directive requirements. In response, in November 2011, the EC sent Poland a justification of its position, once again calling for immediate actions to address the implementation failures. In doing so, the EC pushed the Polish government to adopt a common and integrated approach to address implementation failures. In late 2010, the Ministry of Agriculture and the Ministry of Environment agreed on a common strategy for NVZs designation for the period of 2012-2015 (source: ministerial correspondence). As in previous years, the regional water management authorities were authorised to designate NVZs in their regions, as well as action programmes for these NVZs.

Different from previous years, however, the Ministry of Agriculture ordered a special analysis at the IUNG to support the designation of NVZs by regional authorities (R10; ministerial correspondence; participant observation). This analysis, based on a model of nitrate outflows from agriculture, designated NVZs in each region of Poland (source: ministerial correspondence) and was published by the end of 2011. There was concern by some policymakers inside the Ministry of Agriculture that scientific expertise from one organisation is not a sufficiently reliable basis for a new designation of NVZs and that the results of expertise from the University of Warsaw should also be taken into account (R10). In addition, regional water authorities sent a series of comments and discrepancies to the IUNG analysis expressing gaps on the use of data and methodological concerns (R10; ministerial correspondence). Specifically, the IUNG analysis was based on the national monitoring data, whereas the regional authorities claimed to have more detailed regional datasets.

Even more scientific reports were produced as a reaction to the call of action by the EC (source: ministerial correspondence). The Ministry of Agriculture commissioned a report from experts within one of the Polish Universities regarding the mathematical modelling of areabased pollution coming from agriculture. In addition, the National Research Institute of Animal Production (subordinated to the Ministry of Agriculture) released an analysis estimating the size of the production unit and the nitrogen content of manure produced in different animals' 
housing systems (IZOO 2013). Both analyses brought additional information to the issue of nitrates pollution from agricultural sources (source: ministerial correspondence).

In an official letter of January 2012 to the EC, Poland expressed its willingness to enlarge previously designated NVZs based on the newest scientific analyses (based on the ministerial correspondence). In addition, the NWMA set up a special working group that consisted of representatives of the Ministry of Environment, Ministry of Agriculture, and scientists whose task it was to work on an exemplary action programme for farmers who are based in NVZs (source: ministerial correspondence). Later that year, regional authorities designated more NVZs based on the analyses of IUNG and the University of Warsaw. However, the area of NVZs was smaller than suggested in the expertise of the IUNG (R10; national regulations on designation). As before, the NWMA coordinated the preparation of the implementation report for the period 2008-2011, which was contracted to the Institute of Meteorology and Water Management and sent to the EC.

The experience of the second implementation period (2008-2012) revealed further alignment of Polish infrastructures of expertise with the EU Nitrates Directive requirements. Greater multi-level cooperation between institutions and greater policy integration was found, which was an outcome of the interventions of the EC. In terms of the culture of expertise, research became more integrated in the policy process and offered both instrumental information about the state of nitrates pollution/use and a conceptual frame for revisions of designated NVZs. At the same time, a vertical relationship between policy and science still dominated, as scientific analyses were financed through policy budgets and commissioned through individual contracts.

\subsubsection{Poland in court over nitrates and water pollution}

The EC was not satisfied with the actions of Poland to address implementation failures of the Nitrates Directive and took Poland to the EU Court of Justice for failing to guarantee that water pollution by nitrates is addressed effectively: too little has been done in the eyes of the EC (European Commission 2013; R14). As this decision was already anticipated by the Polish government a year earlier, a discussion at the policy level started regarding the NVZs designation approach (source: ministerial correspondence). At this time, in 2012, a separate department for water resources was restored back at the Ministry of Environment to 
supervise the activities of the NWMA and to formulate national water policy (R15). The Ministry of Environment suggested a complete change of approach from multiple distinct NVZs to one single NVZ for the whole territory of Poland (ministerial correspondence; R15; R10). This was criticised by the Ministry of Agriculture who argued that there is no scientific evidence suggesting the designation of the whole country as one NVZ and it would not guarantee avoiding a court proceeding (R10; R15). As the two Ministries disagreed, the decision was transferred to the Council of Ministers at the Polish Parliament (source: ministerial correspondence).

To support the argument for multiple NVZs, the Ministry of Agriculture delivered the results of a cooperative analysis (ordered late 2012 at its Economic Research Institute) regarding costs for both scenarios of designation (multiple zones vs one zone) (R10). Shortly after, the Ministry of Environment proposed an alternative policy solution: to establish one action programme for the whole territory of Poland limiting outflows of nitrates from agricultural production. This solution did not require designation of any distinct zones. The solution was accepted (ministerial correspondence; R10). In June 2013, the Ministry of Agriculture organised a meeting with scientists from its research institutes to discuss further steps. There was a need to appoint in advance research institutes and scientists who would be able to provide expert support in formulating one action programme for the whole country (source: ministerial correspondence).

After Poland was taken to the EU Court of Justice, scientists became more active and organised a few seminars to inform the general public about the challenges of the Nitrates Directive's implementation and about discharges of nitrates and phosphorus from different sources ${ }^{6}$. Policy officers from ministries and regional water authorities were also invited to these seminars (R10; R15). This was a change in practice, as before scientists had organised seminars principally for other scientists or to report to the Ministry about their research progress. On top of this, the Ministry of Agriculture, the IUNG, and other research institutes organised a public campaign in 2014 to reduce fertiliser usage, which was a prominent example of joint action and cooperation (source: participant observation).

\footnotetext{
${ }^{6}$ This information was collected via analysis of websites of ministerial research institutes who kept track about their own scientific conferences and themes, and also by seeing invitations to the seminars within the ministerial correspondence.
} 
In sum, interventions by the EC had an impact on the communication process at the policy level in which we found that environmental administrations - including the associated infrastructure of expertise - had a stronger position vis-'a-vis the agriculture sector. All the years dedicated to the implementation of the Nitrates Directive had developed a strong policy need to produce scientific knowledge that could be used in different ways in discussions between Poland and the EC and also at the national level.

\subsubsection{New pathway of implementation?}

A judgement of the EU Court of Justice (Court Case C-356/13) forced the Polish Government to take corrective actions towards the implementation of the Directive. Accordingly, a special expert group has been established to finalise a single action programme for Poland and provide proposals for legislative changes (ministerial correspondence; R10; R15). The Ministry of Agriculture seconded key scientists from different scientific institutions who could provide relevant expertise to work in this expert group (R10).

The initiation of corrective actions coincided with a change in the Polish parliament following general elections in October 2015. The new government that was established shortly after introduced many structural changes in the country's institutional framework, including a move back to a mode of centralised government. We believe that these changes will affect the competences of water management authorities at regional and national levels and shift responsibility for the single action programme from regional authorities back to the competences of Ministries (based on the new amendments to the National Water Act of 18 July 2001). All these changes may lessen the involvement of regional authorities into the implementation and policy processes regarding nitrates and may partly undo freshly established practices of cooperation developed during the implementation process for the Nitrates Directive.

\subsection{Discussion: Policy convergence and the science-policy interface}

Our analysis has demonstrated that the implementation of the Nitrates Directive in Poland started from a situation of clear misfit, where institutions and cultures of expertise were misaligned with the requirements that the EU Nitrates Directive set out in terms of institutional cooperation, information infrastructures, and cultures of knowledge production 
and use. The competition between sectors (agriculture vis-à-vis environment) was an additional obstacle to align with the Directive requirements, especially in the early phases of the implementation process. Such competition is found in many member states across the EU (compare Behagel and Turnhout 2011 for the Netherlands). However, ministries of environment in CEE countries used to be especially politically and administratively powerless, this being a heritage of previous political systems (communism, socialism) (Kramer 2004). Subsequent years showed increasing policy convergence in the sense of a development of the necessary institutional cooperation for environmental monitoring and assessment and a strengthening of the position of environmental administrations. The culture of expertise proved to be a more persistent obstacle to meeting EU requirements for a long time, as the interactions between science and policy were still very unidirectional - being steered by policy 'pull' rather than research 'push' (Bielak et al. 2008) - and information was not readily shared. Our analysis has pointed to instances of further convergence in terms of a culture of expertise and a more interactive science-policy interface, but it is difficult to make predictions on how 'deep' or 'shallow' this change will prove to be.

Knowledge and expertise are an integral part of institutional and cultural processes of convergence of national policy with EU requirements. We noticed that institutional and cultural change accompanied each other more often than not, which confirms the idea that these types of change are mutually constitutive, as also expressed by works of Halfmann (2003; 2005) and others. The institutional change of 2006, which included the creation of a new agency to implement the Nitrates Directive, became more pronounced and incorporated social practices of data sharing in the years that followed. Institutional changes towards more policy integration and multi-level cooperation were accompanied by a stronger recognition of policymakers for the need of support by science. Moreover, knowledge was produced by different agencies and used in different forms, depending also on the policy need: instrumental for assessing actual problem; symbolic to build argumentation and legitimisation; and conceptual for unified methodology of NVZs designation. Importantly, personal networks of policymakers and scientists, as well as more structured interactions, also developed over time.

Our case shows that compliance with the requirements of the EU environmental acquis by member states is at least as much steered by the calls for expertise that EU requirements 
imply as it is by more direct interventions by the EC. Specifically, our case showed that certain institutional and cultural changes were direct responses to the nature of the EU law in terms of the type of expertise on nitrate pollution that it requires (see also Kružíková, 2004). The direct interventions by the EU and this more structural need for new 'infrastructures of expertise' (Radaelli 1999) are nonetheless intimately related. Thus, 'doing too little' and being taken to the EU Court of Justice for failures in the Nitrates Directive implementation related to very specific requirements spelling out the need for scientific analyses, a deliberative approach, ensuring diminishing $\mathrm{N}$ concentration from agricultural sources, detailed monitoring, and forecasting. As such, infrastructures of expertise play a key role in policy convergence processes.

Finally, our case shows a paradox. Results point towards the conclusion that policy convergence can be closely tied to top-down pressures from the EC. That is, only through active interventions and pressure of the EC did the cooperation between science and policy move towards convergence over the years. The Nitrates Directive and Poland are no outliers in this respect, Behagel and Turnhout (2011) show how similar knowledge requirements called for by the EU Water Framework Directive had the Netherlands equally struggling to align scientific expertise with policy implementation programmes. At the same time, adaptations in policy culture are intended to bring about a more horizontal and bottom-up type of policymaking.

\subsection{Conclusions}

Our case study demonstrated that, in the context of Europeanisation, it is worth focusing on the science-policy interface as it contributes to policy convergence and compliance with the EU environmental system of governance. Specifically, in the field of environmental policy, science and expertise are not only key aspects of the requirements that EU directives set out, but they are also integrally linked to institutional frameworks and policy cultures (Demmke and Deakin 2001). We have shown that science-policy interfaces are important because EU implementation processes target them directly by calling for the assignment of environmental zones, identification of pressures, and measurable and verifiable actions. All of these actions require the cooperation of policymakers and scientists, in one form or another. Thus, they are part and parcel of the EU's 'acquis communautaire' and therefore warrant empirical analysis, 
as part of EU studies. By offering an empirical, historical example of the process of policy convergence in Poland our analysis contributes to understanding how the implementation of the EU law calls for changes in the science-policy interface as part of the EU system of governance (Bulmer 2008).

Historically, EU environmental policy has been characterised as a top-down, technocratic approach to decision-making (Turnhout et al. 2015). Moreover, Europeanisation processes, in general, are portrayed as top-down processes within EU studies, especially in the context of CEE countries (Carmin and Vandeever 2004; Leventon 2015). We argue that this top-down dynamic gives a specific type of impulse for knowledge and expertise demands within the science-policy interface. In our case study we observed a clear research demand, where policymakers sought knowledge, were prepared to act, and approached their own trusted and credible research institutes directly. Especially in the top-down approaches, there is always a risk that once pressure is gone or political dynamics change, institutional and cultural changes may reverse (Shiers et al. 2014). Recent political changes at the national level in Poland appear to point to such a reverse direction of policy change. We should note that the Nitrates Directive is considered a 'second wave' directive compared to the Water Framework Directive that is a 'third wave' directive (Aubin and Varone 2004). These more recent directives are part of what some call the 'participatory turn' in EU policy (Saurugger 2010), which includes a more active call for stakeholder participation and transparency in reporting, amongst others. Even so, such calls for participation oftentimes remain understood as a top-down requirement, i.e. as the obligation to organise participation and transparency (Behagel and Turnhout 2011). This is a reminder of the entwinement of institutions and policy cultures: it is very hard to change one without the other.

Finally, literature on the EU identifies strategies such as 'researcher push' and 'policymaker pull' to create demand for research and to close the 'know-do' gap (Bielak et al. 2008; Van Kammen, De Savigny, and Sewankambo 2006). Ideally, a balance between policy pull and research push, or supply and demand, would make science-policy interfaces more productive in policy implementation processes (Sarewitz and Pielke 2007; Young et al. 2014). However, in our case study the demand for science - so policy pull - was obvious, less was reported on researcher push. Further research could investigate what conditions would make a 'researcher-push' become part of Europeanisation processes. Such research should 
moreover also focus beyond CEE counties and compare the science-policy interface of the EU with empirical data from other countries and the EU itself.

\subsection{List of interviews and participant observation}

List of Interviews:

- R1: A policy officer involved in the Nitrates Directive implementation process of the

- Ministry of Agriculture and Rural Development, Poland. Interview, December 2008.

- R2, R3: Regional policy officers responsible for the Nitrates Directive in their region, Regional Water Management Authority, Poznań, Poland. Interview, December 20008 .

- R4, R5: Policy officers responsible for monitoring of waters at the national level, preparing monitoring network for the Nitrates Directive, commenting on national reports of the Nitrates Directive implementation, Chief Inspectorate for Environmental Protection, Poland. Interview, January 2009.

- R6, R7: Regional policy officers responsible for the Nitrates Directive in their region, Regional Water Management Authority, Warsaw, Poland. Interview, April 2009.

- R8: A researcher preparing a scientific report about Poland for the European Commission, Wageningen University and Research Centre, the Netherlands. Interview, July 2009.

- R9: A researcher doing scientific analysis on nitrates for the national administration in Poland, Institute of Land Reclamation and Grassland Farming, Poland. Interview, August 2009.

- R10: A policy officer involved in the Nitrates Directive implementation of the Ministry of Agriculture and Rural Development, Poland. Interviews, April 2010 and December 2016.

- R11: A policy officer responsible for national coordination of the Nitrates Directive, National Water Management Authority, Warsaw, Poland. Interview, May 2010.

- R12, R13, R14: Policy officers responsible for controlling EU member states from implementing the Nitrates Directive, European Commission, DG Environment. Interview, May 2013.

- R 15: A policy officer responsible for the Nitrates Directive implementation in Ministry of Agriculture and Rural Development. Interview: September 2016. 
Participant observation:

- November 2011: presence of the first author at the meeting at the Ministry of Agriculture and Rural Development during which scientists from Soil Science and Plant Cultivation Institute presented their analysis - different alternatives towards designation. Following this meeting, two informal phone conference calls between policy officers and scientists took place, during which the first author was present.

- Mid 2014: presence of the first author at the opening meeting of the campaign - stop fertilisation (Ministry of Agriculture and Rural Development together with Institute of Soil Science and Plant Cultivation and Extension services launched this campaign). 
CHAPTER 3

\section{THE USABILITY OF SCENARIO STUDIES: THE CASE OF EURURALIS FROM THE USERS' PERSPECTIVE}

This chapter has been published as:

Kowalczewska, K., and Turnhout, E. (2012). The usability of scenario studies: the case of EUruralis from the users' perspective. Polish Sociological Review, (177), 91. 


\section{ABSTRACT}

Scenario studies are seen as useful tools to support planning and decision-making processes because they provide integrated projections of future trends and developments and their impacts on land use. They play an important role in facilitating cooperation and interaction at the science-policy interface. This chapter contributes to new understandings of the role of science-based tools and instruments such as scenario studies at the science-policy interface. It uses a theoretical framework that connects the criteria of credibility, salience, and legitimacy to the concepts of co-production and boundary object to analyse the EUruralis scenario study that addresses the future of agriculture and rural development in Europe. The findings demonstrate that aspects related to legitimacy contributed to the capacity of EUruralis to function as a boundary object between the scientists and policymakers involved. They also show how cooperation in the EUruralis project resulted in joint learning and reflection. The chapter concludes by discussing the role of EUruralis as a boundary object and connecting the findings to the concept of co-production.

Keywords: EUruralis; boundary object; credibility; legitimacy; salience; co-production 


\subsection{Scenario studies and the science-policy interface}

Scientific knowledge plays an important role in current national and international environmental governance, planning and decision making. Particularly knowledge that pertains to future developments and trends is considered important as a basis for land use planning issues. Scenario studies are considered to be particularly useful in that respect because they are able to project the impacts of land use changes and illustrate future needs and issues in a complex context (Clavel et al. 2011). According to Kok (2006, p. 264), scenarios function as "plausible, challenging, and relevant stories about how the future might unfold that can be told in both words and numbers". In a similar vein, Kahn and Weiner (2000, cited in Kok 2006) state that scenarios are hypothetical sequences of events constructed for the purpose of focusing attention on causal processes and decision-points. Scenarios integrate existing knowledge about drivers and impacts of environmental and land use change. As such, they can contribute to knowledge utilization and to informed decision making in the face of uncertainty.

Several studies have highlighted the important role of tools and instruments such as scenario studies, models, maps, multi criteria analyses, or indicators at the science-policy interface (Turnhout 2009; Sterk et al. 2009; Stirling 2006; Hessel et al. 2009; Clavel et al. 2011). These tools and instruments are considered useful because they are science-based, which lends them a certain epistemic authority, while at the same time, they generate knowledge and information that is considered relevant for policy and decision making. By combining scientific validity and user relevance, these tools are considered crucial in bridging the gap between science and policy and enhancing knowledge utilization. However, their capacity to play such a role in improving the science-policy interface depends on the extent to which they are able to meet the demands of the users (Sarewitz and Pielke 2007).

This chapter explores these demands by offering an analysis of user perspectives on the usability of scenario studies. It uses the EUruralis project as a case study. The EUruralis project is a scenario study that addresses the future of agriculture and rural development in Europe. The project, which started in 2004, has been initiated by the Working Group of Sustainable Development and System Innovation set up at Wageningen University and Research and commissioned by the Dutch Ministry of Agriculture, Nature and Food Quality (LNV). The 
EUruralis project uses several scenarios to forecast the possible future of rural areas in the light of the current reforms of the Common Agriculture Policy (CAP). It was the explicit ambition to make EUruralis useful for policymaking in EU member states and to provide topics for debate on long-term rural land use and agriculture (Rienks et al. 2008). An important feature of EUruralis was that it involved policymakers in the project. Our analysis focuses on the perspectives of Polish scientists and policymakers on the usability of EUruralis. Poland was one of the countries in which EUruralis was introduced to enable Polish policymakers to use it while discussing the current rural development and agricultural issues. The introduction of EUruralis involved several meetings in the Polish Ministry of Agriculture and Rural Development and in the Netherlands, for example in Alterra Research Centre, where Polish policymakers as well as scientists who were asked to verify the data input and the first results of the prognoses in the EUruralis version 1.0, study EUruralis, and ask questions to the designers about the project. Currently, EUruralis is not used in Poland. Our analysis is based on relevant policy documents and literature as well as on material from semi-structured interviews and questionnaires. Ten out of thirteen interviewees were with the prospective users of EUruralis: Polish scientists and policymakers. The other three interviewees were Dutch representatives involved in the design of EUruralis. In addition, fourteen questionnaires were sent out and five returned. The analysis focused on the assessment of the usability of EUruralis, the reasons and criteria that were mentioned, and the characteristics of the sciencepolicy interactions that took place in the context of the project. Also, respondents were asked to reflect on the EUruralis project itself, on the processes of cooperation and interaction involved, and on how this project influenced their practices. Before presenting the results of the analysis, the next section introduces the theoretical framework used in this research.

\subsection{Usable knowledge}

Connecting science and policy is considered important to improve planning and policy decisions and underpin them with a scientific basis. However, the science-policy interface is often characterised by communication problems and other difficulties. (e.g., Johnston and Soulsby 2006; Turnhout, Hisschemöller, and Eijsackers 2007; 2008, Sarewitz and Pielke 2007). On the one hand, civil servants can exhibit a tendency to believe that their experiences are unique and better than those of others. On the other hand, scientists often perceive policymakers as science enemies who are not interested in their results (Pielke 2007). 
Moreover, the separation between science and policy is also considered important. Scientist must be able to maintain their independence from policy in order to be seen as neutral and objective, policymakers must be able to show that they are the ones who are in charge and not the scientific experts (Huitema and Turnhout $\left.\begin{array}{lllll}0 & 0 & 0 & 9\end{array}\right)$. Thus, the use of knowledge in policy and decision making cannot be assumed.

In light of these difficulties in connecting science and policy, it is not surprising that many authors claim that knowledge is often not used effectively (e.g., Meffe 1998; Bradshaw and Borchers 2000; Lawton 2007; Pohl 2008). If this situation is to improve, the production of usable knowledge should be enhanced. But what constitutes usable knowledge? Various authors in the field of knowledge utilization studies have come up with partly overlapping sets of criteria for usable knowledge (e.g., Rich 1991; Weiss 1995). Part of these criteria focus on the quality of knowledge. It is assumed that for knowledge to be usable, it should conform to scientific standards related to reliability and accuracy and it should be based on state-of-the-art methodologies. In practice, experts involved in the production of usable knowledge tend to focus a large part of their efforts on technical issues and on enhancing the quality of knowledge (Turnhout, Hisschemöller, and Eijsackers 2007). However, this is only part of the equation. As Lindblom and Cohen (1979) have pointed out the usability of knowledge is not only determined by its quality, but also needs to consider demands of users. Usable knowledge is relevant for users if it is delivered timely and if it is related to topics that are currently salient and that they are working on (Weiss 1995). Second, it has been demonstrated that knowledge users assess knowledge based on their prior knowledge,

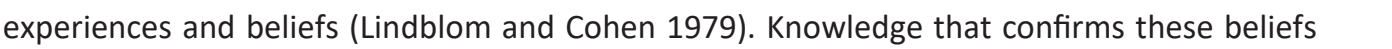
has a much greater likelihood of being accepted as usable than knowledge that contradicts them. Finally, problem and action orientation are important for usable knowledge (Weiss 1995). From that perspective, interdisciplinary knowledge based on an integrative approach that addresses the different dimensions of a current societal and environmental problem is considered to be more usable than monodisciplinary knowledge (Sumner 2003), amongst others because it can be translated into realistic and effective strategies for action to address these problems.

Meeting the different criteria for usable knowledge is no simple matter. Especially relevance and quality are often considered to involve a balancing act, because the quality of knowledge 
is assumed to depend on distance from policy, which will decrease the chances of producing knowledge that is relevant (Huitema and Turnhout 2009). Furthermore, the question which knowledge is considered usable crucially depends on the context in which it is developed and applied. Recognizing this implies conceptualizing the relation between knowledge production and use not as a 'chain of knowledge' from knowledge production - by science - to use - by policymakers - but as a dynamic science-policy interface (Turnhout, Hisschemöller, and Eijsackers 2007). In other words: "usable knowledge is co-produced in the context of every - day interactions between science and policy and the public" (Lemos and Morehouse 2005, p. 59).

In that light, the contribution of Cash et al. $(2002 ; 2003)$ is interesting. They propose three main criteria: credibility, salience, and legitimacy, which are explicitly considered as socially negotiated outcomes rather than essential characteristics of knowledge. Credibility implies that knowledge is seen as realistic and valid. This refers to perceptions of scientific quality - including the methods used and the reputation of the knowledge producers - as well as to aspects related to the completeness and transparency of a study. Salience refers to the relevance for a user, including the timing of knowledge and the link to decision-making agendas, or to choices an actor has to make. Salient knowledge is typically problem oriented. Often this requires a multidisciplinary approach to generate an integrated perspective of the phenomenon under study. Legitimacy is the most process oriented of the three. It refers to the fairness of the knowledge production and use process, including questions of the interaction, cooperation, deliberation and participation.

Cash et al. (2003, p. 8090) recognise that trade-offs and overlaps exist between the criteria and that they are ambiguous and subject to multiple potentially competing interpretations: "each actor [is] likely to enter the debate under different concepts of what makes information salient, credible and legitimate". Consequently, they argue that processes of communication and interaction are required to achieve acceptable levels of all three criteria. In such an interactive multi-actor approach, knowledge may perform a role of a boundary object. Star and Griesemer (1989, p. 393) have described the concept of boundary objects as "an analytic concept of those scientific objects which both inhabit several intersecting social worlds [...] and satisfy the informational requirements of each of them [...]. They have different meanings in different social worlds, but their structure is common enough to more than one world to 
make them recognizable, a means of translation. The creation and management of boundary objects is a key process in developing and maintaining coherence across intersecting social worlds". Thus, the concept of boundary objects implies that usable knowledge allows for different interpretations and meanings in different social worlds as well as meets the demands of all those involved. Their role in facilitating communication across differences is often enhanced by the common vocabulary that boundary objects provide (Carlile 2002; Turnhout 2009).

In this chapter we use analyse users' perspectives of the credibility, salience, and legitimacy of EUruralis. Subsequently, the analysis provides a basis for further discussion of EUruralis in relation to the concept of boundary object.

\subsection{Introducing the EUruralis project}

EUruralis is a computer-based scenario model that presents forecasts of the possible future of rural areas in Europe while considering the current reforms of the CAP. It is a discussionoriented tool to support policymakers in discussions about the future of agricultural and rural areas with scientifically sound data and methodologies for Europe in detail and with the global dimension becoming more important. An important feature of the EUruralis tool is that it links economic and biophysical domains and global and local scales (The EUruralis homepage, 2011). It has three versions. Whereas version 1.0 was released "to give an impulse to the discussion on rural development in the EU-25" (The EUruralis homepage, 2011) and presented forecasts on the country level, version 2.0. offered prognoses on the level of the European regions, had improved interactivity and included EU policy options on the future of the CAP and on biofuels. Version (3.0.) includes a new policy option, i.e. Reducing Emissions from Deforestation and Degradation policies (REDD) and a biodiversity indicator (see the EUruralis homepage for an overview).

EUruralis is based on the DPSIR approach which distinguishes between "driving forces (D) (either direct or proximate or indirect or distant) affecting a defined system (ecosystem, agro-system) by so-called pressures (P) affecting its state (S). This can be seen as the impact, which has to be assessed from society's interests (negative or positive, acceptable or unacceptable). This assessment can lead to policy interventions (Response: $R$ ). These can be targeted at effects (mitigation, compensation) or - more fundamentally - at the direct or 
indirect drivers". (Klijn et al. 2005, p. 36 ). It also addresses the People, Profit and Planet dimensions of land by recognizing that land use is "on the one hand the expression of societal needs, interest, economical laws, (Profit), techniques and on the other it exerts direct influences on the biophysical and partly socio-cultural values (Planet, People)" (Klijn et al. 2005, p. 36).

EUruralis includes four scenarios, which are derived from the IPCC-SRES ${ }^{7}$ scenarios of global coverage (Klijn et al. 2005, p. 52). The two A - scenarios focus on the international cooperation - regional vs. global. The two B - scenarios focus on government intervention -extensive vs. limited. Scenario A1 "assumes multilateral cooperation on economic issues [...], societies are driven by market-based solutions resulting in high economic growth rates" (Klijn et al. 2005, p. 53). Scenario A2 "assumes a view that social and cultural values can best be pre- served in regional political alliances, within which nation states should keep as much sovereignty as possible. No further enlargement of the EU will take place" (Klijn et al. 2005, p. 54). Scenario B1 "assumes multilateral cooperation on issues aiming at fair distribution of wealth, social justice and environmental stewardship. Trade barriers are gradually removed" (Klijn et al. 2005, p. 54). Scenario B2 "assumes that social and cultural values can best be preserved at the community level. [...] self-reliance, environmental stewardship and equity are the keys to sustainable development. Government intervention is necessary to facilitate negotiations between stakeholders and enforce decisions" (Klijn et al. 2005, p. 54).

The basic input for the scenarios is provided by three models: LEITAP, IMAGE, and CLUE (Klijn et al. 2005). LEITAP is an economic model, which is adapted from the Global Trade Analysis Project (GTAP). It aims "to support quantitative analysis of international trade, resource and environmental concerns in economic wide framework" (Klijn et al. 2005, p. 63). IMAGE (Integrated Model to Assess the Global Environment) assesses the effects of global changes in population, agricultural production and climate on the environment. It "supports decisionmaking by quantifying the relative importance of major processes and interactions in the society-biosphere-climate system" (Klijn et al. 2005, p. 77). CLUE (Conversion of Land Use and

\footnotetext{
${ }^{7}$ Intergovernmental Panel on Climate Change-Special Reports on Emissions Scenarios.
} 
its Effects) is a land use model that allocates changes in the land use and "was developed for the spatially explicit simulation of land use change" (Klijn et al. 2005, p. 83).

EUruralis was designed in an interactive way and involved various stakeholders throughout the process. The initiative itself arose from a joint discussion with policymakers and scientists about the possibilities of developing a model that would provide projections on the development of rural areas and agriculture in Europe. The involvement of scientists and policymakers is also affirmed with the establishment of two groups. The Policy Advisory Group consisted of civil servants and gave advice on the policy relevance and orientation of the scenarios as well as on the presentation mode of EUruralis (Klijn et al. 2005). The Scientific Advisory Group consisted of representatives from Environmental Assessment Agency (RIVM), Agricultural Economics Institute (LEI), Wageningen University, and UNEP-GRID ${ }^{8}$ in Poland and INRA-CIRAD ${ }^{9}$ in France. The groups cooperated in the formulation of research questions, the building of the models, and the verification of the results. The EUruralis project members invited amongst others scientists from the Polish Institute of Agriculture and Food Economics and Polish Institute of Geography and Spatial Planning of the Polish Academy of Sciences to comment on the results regarding the prognosis for Eastern and Central Europe.

\subsection{User Perspectives on the usability of EUruralis}

This section discusses the results of our analysis in terms of the three criteria of credibility, salience and legitimacy.

\subsubsection{Credibility}

One of the aspects that spoke to the credibility of EUruralis is its multidisciplinary. Polish respondents emphasised that EUruralis aggregates many disciplines and achievements of science. This allows for multidisciplinary analyses, helps to define, identify and understand complex situations, and promotes new ways of thinking about and dealing with them. This multidisciplinary approach is assured by the three-model-construction [LEITAP, IMAGE, and CLUE (Klijn et al. 2005)] as well as by using the DPSIR and sustainability approaches. EUruralis

\footnotetext{
${ }^{8}$ United Nations Environmental Programme-Global Resource Information Database.

${ }^{9}$ Institut national de la recherche agronomique-La recherche agronomique pour le developpement.
} 
addresses people, planet, and profit (the three Ps) issues, and includes knowledge about ecological properties, economic aspects and socio-cultural values from a wide variety of scientific disciplines including economics, ecology, climate science and land use studies. By integrating multiple dimensions and knowledge from multiple disciplines and sources, EUruralis is considered to be credible and able to attract a broad audience.

The usage of the DPSIR approach and particularly the way it includes outside factors as driving forces and pressures contributes to its outcomes being seen as realistic. Examples of issues, which happened outside Europe but influence the CAP and the daily life and future of Europe's citizens include the growing population and income in such countries like China, India, and Brazil; the international trade barriers and opportunities; oil production and global change processes. So, in EUruralis forecasts' drivers such as: natural forces (climate change, changes in sea level), geopolitical change and international trade, demography, world economy, technology development, consumer behaviour and policy considerations are taken into account (Klijn et al. 2005).

The way EUruralis presents its results (historic and temporary facts, figures and pictures of the EU 27), in an understandable manner by using maps, graphs and tables and in four contrasting scenarios, also contributes to its credibility. EUruralis designers compared it to the commercial games like SimCity and SimRural PC games because "it is a challenge for users to find themselves new reasons and manners for land use changes on the territory of the EU 27 ". Polish respondents indicated that they appreciated this way of presenting the results because it makes it much more attractive than a traditional scientific publication. They emphasised "the easiness of getting the results of prognosis" and they spoke about the simple and colourful maps, graphs, tables which made the project understandable for anyone. Generally, Polish respondents recognized that the way in which EUruralis presented the results contributed to the usefulness of research in policymaking.

Nevertheless, the credibility of EUruralis was also criticized. One of the weak points concerned the lack of continuous data from the past years from Eastern and Central Europe used in equations calculating the forecasts. This resulted in incorrect prognoses of changes in some regions, including Poland. Polish respondents were very critical about this, and one of them said: "if the results are not correct, this disqualifies a model and it cannot support 
policymakers". In the eyes of Polish interviewees, this strongly weakened the validity of EUruralis and influenced its utilisation by potential users in Poland.

\subsubsection{Salience}

EUruralis was intended to serve as a tool to support discussions about policy issues and possible actions at the European level. The way of presenting the results in contrasting scenarios was appreciated because it linked up with current policy issues. Responses to version 1.0 made clear that forecasts on a country scale were not considered to be very relevant and usable. Several scientists and policymakers expressed during interviews that they would prefer region specific information. Version 2 .Orectified this. According to a scientist working for the project these changes improved the relevance of EUruralis: "[EUruralis] has been improved by showing the data at the regional level". The salience of EUruralis was also enhanced by adding policy options that constituted hot issues on the international rural arena (CAP market and income support policy, bio-energy and less favoured policy). It was assumed by the designers that the 2.0 version could be relevant mainly to civil servants, NGOs, farmer associations, environmental and nature groups, colleagues in research and for purpose of educational workshops.

Apart from appropriate data resolution scales, the salience of EUruralis lies partly in its capacity to help decision makers in setting priorities. As one of the respondents explained:

there is a long list of issues [...] in the context of rural development such as water, soil, climate changes, employment in rural areas, innovations etc. But policymakers are looking for a shortlist of issues to be discussed. So EUruralis helps them to make this list. [...] It contributes to putting together a shortlist of important issues that should be changed or embedded in the European law.

One particular aspect of salience is problem orientation. The DPSIR approach mentioned earlier contributed to this as well as the multidisciplinary approach of EUruralis and its use of sustainability indicators. It also used so-called 'meta indicators' that integrate scores for the three Ps for specific countries and regions and assess differences between, amongst other, east-west, north-south, EU 15 and EU 10. This was purposively done to generalize results and present them in a policy relevant way that would be understandable for inexperienced users (Klijn et al. 2005). According to several respondents, the prognoses of changes based on societal, economic and environmental indicators and the combination of economic and environmental models enabled them to look at issues from a broader perspective. Apart from 
problem orientation, action orientation is important for salience as well. Respondents appreciated the integrated character and future orientation of the scenarios that provide forecasts on what may happen in agriculture and rural areas in Europe in ten-year intervals until 2030. As such, EUruralis facilitated discussions on policy issues regarding the effects of the accession of new member states and the implementation of environmental directives on land use.

The set of scenarios contribute to saliency as well because one can "analyse [for example] how driving forces can influence biodiversity and ecosystems" (Verboom et al. 2007). "The set of scenarios enabled also discussion of policy relevant issues and contextualized the user towards broad issues of globalization, cultural identity, environmental awareness and international solidarity, which are typically at the roots of long term scenario studies" (Klijn et al. 2005). As noticed by Verboom et al. (2007) such scenarios help in making alternative assumptions, beliefs, and attitudes explicit, and illustrate how they influence future conditions. The scientists involved in the design of EUruralis admitted that "perhaps, scenarios are overlapping, but if things are happening in all four scenarios than there is high possibility of a true prognosis. If things are happening only in one scenario, it is probably some kind of extreme". Also, most of Polish interviewees recognized the scenarios as a positive feature; whereas, one Polish scientist understood this as limiting the number of options and stressed that "it is not possible to fully predict possible changes in the project if they are treated linearly because in nature there are no linear events". Polish respondents expressed their curiosity and interest in the EUruralis results projected in the time horizon and admitted that it contributed to their knowledge. In that sense they indicated that EUruralis is policy and action oriented.

\subsubsection{Legitimacy}

Although EUruralis involved stakeholders and was introduced in several countries, it was mainly Dutch scientists that were involved in its design. Particularly in the early stages of the EUruralis project, participation of actors from other countries was limited. One of the respondents indicated that this was a weak point of the project and that it would have been better to include a broader group of actors (scientists and policymakers) from various countries. 
During the project, interaction increased. There were many informal contacts between policymakers and scientists involved for example by phone, emails. As one of the respondents recalls:

during the preparation of the EUruralis version 2.0, there were many informal working meetings in the Netherlands, where policymakers and scientists met. In fact, the majority of these meetings took place outside the Dutch ministry, in scientific organizations, where policymakers felt they could discuss issues more openly.

The interactions between the various actors concerned amongst other discussing policy options and preferences, translating policy questions into model equations, and experimenting with different ways to visualize and present the results. Although these processes required interaction, they were largely science-based with policymakers assisting the process by providing feedback and specific inputs. Interestingly, EUruralis itself enhances interaction as well. Users can choose between different domains and levels of information and they can explore different options and the implications for rural areas. It thus offers possibility to create relations between different domains and levels of information.

The cooperation between policymakers and scientists in the project was generally appreciated by the respondents. They felt that it contributed to bridging the gap between policy and science. Moreover, they felt that EUruralis encourages dialogue between policymakers and scientists on the possible implications of changes for policy by offering a common terminology and vocabulary. Cooperation in EUruralis provoked reflection on the differences between science and policy. As one of the respondents explains:

scientific and policy domains differ enormously and it is important that everybody has an open mind. It is not possible for all scientists to understand what policymakers need and vice versa. I think in general that policymakers overestimate the impact of policy and tend not to look on the real long term and also broader then their own region or country. [...] Scientists focus mainly on their own topics and tend to keep on modelling.

Reflection on the differences between the domains also implied recognition of the importance to overcome them. However, this was not considered to be easy. According to the respondents, useful interactions were possible but they require a lot of work. As one of them explains:

[achieving a productive] science-policy interface requires a lot of effort. In the EUruralis we have invested a lot, but even then it was not always successful. Other research projects have to invest a lot more into making the science-policy interface more successful. 
According to the respondents, cooperation in the project not only contributed to the awareness of how science and policy differ also to an increased appreciation of the actor's different perspectives. The scientists involved were happy to learn that policymakers were interested in models and results. As one of the respondents states:

I found that policymakers are also specialists. We spoke with policymakers from ministries of agriculture and rural development. They were very much interested in detailed impact assessments of rural development policy.

Another respondent admitted to have learned a lot for example about the importance to explicitly address issues of relevance, and to present forecasts to policymakers in an understandable way. The policymakers involved increased their understanding of the various complexities involved in modelling and realized that scenarios and prognoses cannot show and predict everything. EUruralis was identified by one of the respondents as:

a good learning experience for scientists of different discipline to cooperate and to connect each other's models and to understand each other's language.

Respondents were asked also to reflect on the project itself, its initial goals and its actual function in practice. Some realized that perhaps the expectations for EUruralis had been a bit too ambitious. Initially, EUruralis was assumed to be a decision support tool not only for policymakers but also for broader audiences. One of the respondents makes clear that this assumption was perhaps misguided: "the model can be used as an instrument by scientists to analyse research questions posed by policymakers. Also it might also be useful for students as a learning tool and that is it". However, this was considered to be very valuable. As one of the respondents explains:

maybe the most important contribution of EUruralis lies not necessarily in the results and prognoses it produces but in the way it facilitates policy discussions that are scientifically supported and deal with important trends and issues in rural areas in Europe.

This shows how during the process, EUruralis came to be interpreted and used in different ways than was originally anticipated.

\subsection{EUruralis as usable knowledge and boundary object}

The previous section has presented different perspectives on the usability of EUruralis in terms of its credibility, salience and legitimacy. The credibility of EUruralis appears to lie primarily in its multidisciplinary, its broad DPSIR approach and its clarity in presenting the 
results. By integrating different domains and knowledge, EUruralis is able to attract a broad audience and is considered credible because it offers a realistic representation of the problems and issues at hand. However, the lack of data was considered to decrease its credibility. The salience of EUruralis appears to lie in its ability to connect with currently salient policy issues related to the future of the CAP, the implementation of EU policies and the implications of these for rural areas and in presenting the prognoses in four contrasting scenarios. The future-orientation of the scenarios and the way they present the results ensure action orientation of EUruralis.

In terms of legitimacy, EUruralis was characterized by an interactive set-up. In order to make EUruralis a relevant and usable support tool for policy discussions, the project aimed at the involvement of users. Scientists and policymakers cooperated in the project and were involved in joint processes of knowledge production. Our findings also show that to a certain extent, science and policy remained separate communities with different objectives and activities. Thus, EUruralis was able to facilitate the interactions between scientists and policymakers but did not completely collapse the two. Still, those involved did not remain unaffected. Cooperation in EUruralis triggered reflection on the differences between science and policy and the importance and possibilities of overcoming them. Additionally, a learning process was involved, which resulted in increased knowledge and appreciation of each other's perspectives.

It has to be highlighted that the respondents valued different things in EUruralis and their perceptions were very differentiated. Whereas scientists focused more on the construction of the scenarios and the calculation and generation of results, policymakers focused on problem and action orientation of EUruralis, plus relevance and its possible usage in their work. For example, the multidisciplinary approach of EUruralis was appreciated for different reasons. Scientists appreciated the scientific challenge of coupling models from different disciplines. Policymakers valued the integrated perspective and the link to action that the multidisciplinary perspective offered. The way of presenting the results was also perceived differently. Scientists presented the results in such a way, including different policy options, in order to enable their use in policy discussion, while some of the policymakers focused on whether the prognoses were true. And this turned out to be an important issue due to the lack of data in some European regions. Finally, perspectives of EUruralis changed over time as 
well. Actors started to recognize that the initial ambitions of EUruralis were perhaps unrealistic and that the current role that EUruralis played in the facilitation of discussions was equally valuable.

Our analysis so far on the perspectives of the credibility, salience and legitimacy of EUruralis makes clear that it was flexible and ambiguous enough to support different interpretations. As a result of this combination of flexibility and stability, EUruralis was able to perform as a boundary object (Star and Griesemer 1989). It meant different things to different people and people had different reasons for wanting to be involved in the project. Still, it was able to satisfy their different needs and facilitate communication and interaction between them. Moreover, our analysis confirms earlier observations about the role of boundary objects in facilitating communication by providing a common vocabulary and offering space for learning and reflection (Turnhout 20009 , Carlile $\begin{array}{lllllll}0 & 0 & 0 & 2 & 0\end{array}$.

However, this role as a boundary object was not present from the start. Our analysis of the legitimacy of EUruralis points to the learning and reflection that took place during the process. The processes of cooperation and interaction involved resulted in the generation of new knowledge and understandings of scenario studies, the recognition and appreciation of differences and the emergence of commonalities and shared interests and perspectives. Its capacity to function as a boundary object was not engrained in the scenario study itself but the outcome of the process. This demonstrates that processes of knowledge production and use are context specific (e.g., Turnhout, Hisschemöller, and Eijsackers 2007). The questions of what will be taken to be usable knowledge or what kinds of knowledge will be considered to be of credible, relevant, action oriented and so on, are context specific. Different people, scientists or policymakers in different EU member states will value different things and will interpret knowledge and science-based policy tools such as EUruralis in different way.

\subsection{The role of scenario studies: co-production in-the-making}

Planning and decision making is an endeavour, which requires an integrated and futureoriented approach. Our analysis exemplifies that scenario studies can in principle contribute to these processes by connecting science and policy. The hybrid character of EUruralis, based on scientific input from different disciplines as well as input from policymakers, facilitated this role at the science-policy interface. 
According to Cash et al. (2002; 2003), knowledge is effective if acceptable levels of credibility, salience and legitimacy are obtained. As Cash et al. $(2002 ; 2003)$ have pointed out, there is the possibility of trade-offs and overlaps between these criteria. Our analysis has demonstrated that the respondents indicated different aspects of what makes EUruralis salient, credible and legitimate. The processes of interaction and cooperation involved in EUruralis project were important in ensuring its legitimacy. However, these processes contributed to its credibility and salience as well.

Thus, interactive and participatory processes that are considered legitimate are instrumental in the production of knowledge that will be considered credible and salient. Not only because the interactive processes affected EUruralis itself, but also because they involved learning and reflection. It was in the context of these processes that EUruralis was able to perform as a boundary object. Thus, our analysis shows how a boundary object such as EUruralis by connecting science and policy also changed them (Van Egmond and Zeiss 2010). EUruralis might be considered as an example of co-production in-the-making (Jasanoff 2004); a situation in which different actors - scientists and policymakers-not only interact and cooperate, but also change their perspectives on science, policy and the relation between the two. Although EUruralis project was not used in Poland as a support tool while discussing the current rural development and agricultural issues, it was a significant learning exercise for both Polish scientists and policymakers. Based on the Dutch practice, they experienced how the interactive development of such multidisciplinary and participatory projects could be organized. However, it remains to be seen whether this will lead to changed practices in science, policy and the science-policy interface of those who were involved in the introduction of the EUruralis project. That is why, we have used the term co-production in-the-making.

To conclude, scenario studies such as EUruralis, which are situated in a policy context and which demonstrate different policy alternatives, are potential examples of usable knowledge, because they are able to bridge the gap between science and policy and link knowledge supply to users' demands. The findings of this study are particularly relevant as the need for such tools in complex and long-term issues such as agriculture and rural development is likely to increase. However, our analysis has also pointed to the importance of designing these processes in such a way that they contribute to the achievement of acceptable levels of credibility, salience and legitimacy. Particularly, the importance of legitimacy should not be 
underestimated as a necessary requirement for facilitating constructive interactions, openness and mutual learning. If this is taken into account, scenario studies have a great potential in addressing complex issues and in stimulating reflection and co- production.

\subsection{List of interviews and questionnaires}

- 6 interviews with employees of the Ministry of Agriculture and Rural Development: April 2007, Warsaw, Poland.

- 2 interviews with employees of the Institute of Agricultural and Food EconomicsNational Research Institute: April 2007, Warsaw, Poland.

- 1 interview with an employee of the Institute of Geography and Spatial Organization, Rural areas study group: April 2007, Warsaw, Poland.

- 1 interview with an employee of the Institute of Land Reclamation and Grassland Farming, Department of nature protection in rural areas: April 2007, Warsaw, Poland.

- 1 interview with an employee of the LNV: May 2007, Wageningen, the Netherlands.

- 1 interview with an employee of the LNV and Environmental Assessment Agency in the Netherlands: May 2007, Wageningen, the Netherlands.

- 1 interview with an employee of Alterra Wageningen University and Research, Department of Landscape-project coordinator: May 2007, Wageningen, the Netherlands.

- 1 questionnaire by an employee of Alterra Wageningen University and Research, Department of Landscape-project coordinator: 2009.

- 3 questionnaires by scientists of the EUruralis team: 2009.

- 1 questionnaire by an employee of the LNV and Environmental Assessment Agency in the Netherlands: 2009. 
CHAPTER 4

HOW TO CREATE USABLE KNOWLEDGE IN A PUBLIC PROCUREMENT CONTRACT: THE ROLE OF A KNOWLEDGE BROKER

To be submitted to a scientific peer-reviewed journal. Authors: Kowalczewska, K., and Turnhout, E. 


\section{ABSTRACT}

Experiences from existing science-policy interfaces call for mechanisms for communication and exchange between scientists and policy-makers that go beyond the 'old' linear supply and demand model. This chapter discusses how the relation between science and policy is organized in a public procurement contract. Our analysis shows that the public procurement contract offered good possibilities for knowledge brokering activities related to the problem formulation, the identification of research needs, and the management of the interactions between the procurer and the researcher. In doing so, the knowledge broker in our case was able to ensure the usability of research. The example of public procurement offers a valuable contribution to science-policy interface studies because it offers a unique way to organize the relation between knowledge production and use, which is demand driven and guided by strict rules. As such, it offers a governance framework that could serve as a model for the organization of effective and legitimate science policy interfaces.

Keywords: science-policy interface, knowledge broker, usable knowledge, public procurement 


\subsection{Public procurement and the science-policy interface}

The expectation of the society in investments in science is that scientific knowledge, and its effective use, will lead to better policies and regulatory decisions (Holmes and Clark 2008). Studies on environmental ministries and regulators in the European Union (EU) member states show their increasing emphasis on the effective use of science in policymaking and regulations (Holmes and Clark 2008; Holmes and Savgard 2008). In fact, national governments and European institutions spend a lot of money on research funding available through different research instruments. One of the instruments that has gained attention amongst EU policymakers is public procurement (PP) (Munns and Bjeirmi 1996; Rolfstam 2009). Many governmental and European institutions, including the European Commission (EC), work intensively with PP contracts to commission a variety of goods, work, and services, including scientific studies (European Commission Vademecum 2013). In the literature, scholars have investigated the workings and effects of the current PP processes (Holzmann 2013; Rolfstam 2009; Koskinen and Makinen 2007; Munns and Bjeirmi 1996; Carayannis and Popescu 2005) in product development, IT industry (Holzmann 2013), E-procurement, and innovation (Rolfstam 2009). This scholarly work provides valuable insights into how PP contributes to the credibility of governments and competitiveness of knowledge-based economies. Our aim in this chapter is to extend and build on these insights by focusing on the procurement of research. Specifically, we are interested in examining how the arrangements within PP can contribute to the production of usable knowledge and thus better inform policy and decision making.

Current scholarship on the science-policy interface has demonstrated that the relation between the production and use of knowledge is not self-evident. Often inadequate communication or other problems result in a failure to bridge the knowledge practice gap (e.g., Johnston and Soulsby 2006, De Koning et al. 2014). Problems may occur due to boundaries between science and policy related to differences in values, in knowledge needs and requirements, and in the interpretation of knowledge differences and different values (Randhawa et al. 2017). In this context, the facilitation of knowledge exchange between science and policy has become essential within organizations (Haas 2015) in order to reconcile the supply and demand of knowledge (Sarewitz and Pielke 2007), and so produce the usable knowledge. 
PP arrangements may play a useful role in this reconciliation since actors from the two sides have to act together. On the demand side there are the policymakers that procure the study. On the supply side, there are scientists or scientifically trained experts in consultancies or research originations. The role of the public procurer is to define the concept, scope and aim of the study and to manage the contract after the supplier has been selected. The management involves the planning, controlling and monitoring of the knowledge production process (Munns and Bjeirmi 1996). The role of the supplier is to carry out the research and deliver results that fit the expectations of the procurer.

Considering the high number of scientific studies commissioned by means of PP in the EU, and especially by the EC itself, one would expect to find in PP contracts good practices of how science and policy can jointly construct usable knowledge. This chapter presents an example of one contract within the PP in which a so-called knowledge broker played an important role in the production of usable knowledge. Knowledge brokers are intermediaries who are able to translate the needs of users into questions that can be addressed by means of research and who are able to facilitate interaction and communication. In doing so, they play an important role in connecting science and policy and ensuring the usability or research (Sverrisson 2001; Bielak et al. 2008; Ward, House, and Hamer 2009; Turnhout et al. 2013). In this chapter, we focus on the role and activities of knowledge brokers to deepen our understanding of the contribution of PP to effective science-policy interfaces.

Before presenting our findings, the next section discusses the concept of knowledge broker and explains our case study and methods.

\subsection{The role of knowledge brokers in ensuring the usability of knowledge}

Knowledge brokers can play an important role in the process of facilitation the exchange between sources of knowledge and users of knowledge that is crucial in order to enhance the usability of knowledge (Bielak et al. 2008; Lomas 2007). The literature has specified different functions, activities and roles of knowledge brokers. Generally, this literature refers to specific individuals either within science or policy, or located in intermediary, or so-called boundary organizations (Boari and Riboldazzi 2014; Saarela et al. 2015) who mediate between science and policy and do not belong to any of these communities. But, for example, studies in impact 
assessment have shown that the users of knowledge (such as policymakers) can perform the role of knowledge brokers as well (Saarela et al. 2015).

There is a broad set of activities that knowledge brokers may perform. According to Meyer (2010) and Ward, House, and Hamer (2009), knowledge brokers facilitate the transfer of knowledge by organizing connections between those who require knowledge and those who can supply it. Knowledge brokers also play a role in the identification of the specific knowledge needs by creating space for the sharing of different interpretations and perceptions about expertise, knowledge and knowledge use (Van Pelt et al. 2014). Overall, knowledge brokers deal not only with the content of a knowledge need but also with the process (collaboration, communication, sharing, and common understanding). They use variety of tools such as faceto-face contacts, meetings (stationary or online), workshops, trainings, and tools such as databases, schemes, templates. "Sometimes they also go beyond creating only connections and take an actual part in creating the knowledge itself while adding to it a supplementary value" (Holzmann, 2013 p. 3; see also Ward, House, and Hamer, 2009; Goffin et al. 2010).

In public procurement context we would expect knowledge brokers to engage in a process of communication and interaction between policymakers and scientists, so knowledge users and producers, where user demands take central stage. Knowledge brokers offer an attempt to help knowledge exchange work regarding what is demanded and what can be supplied better for the benefit of all involved (Bielak et al. 2008). Their work can involve indeed a broad range of activities, amongst others, translating policy problems into researchable questions, assisting people to understand each other's abilities and needs, guiding people to sources of knowledge, helping build links, bringing people together (Bielak et al. 2008).

Different scholars propose different conceptualizations for knowledge brokering activities (Turnhout et al. 2013; Michaels 2009; Meyer 2010; Ward, House, and Hamer 2009; Oldham and McLean 1997; Bielak et al. 2008). Table 2 synthesizes this literature for a general overview of types of activities that can be expected and identifies two categories of knowledge brokering activities that are relevant for the interactions between knowledge production and use in the case of PP. The first category is related to what we call 'translation'. It involves activities such as formulating and informing of policy issues, translating policy issues into research language, sharing and disseminating useful sources of knowledge (relevant research, 
websites, factsheets), transcoding evidence into different vocabulary (understandable for policymakers), and/or making arrangements for different people to meet so as they could together identify knowledge gaps. The second category we called 'flexible intermediary' because practices refer to the management of the interaction processes between different groups or individuals. Activities may involve designing a good process of interaction, facilitating communication, helping to build links, developing positive relations, ensuring that misunderstandings are being clarified, and/or building trust and a cooperative attitude of all involved. 


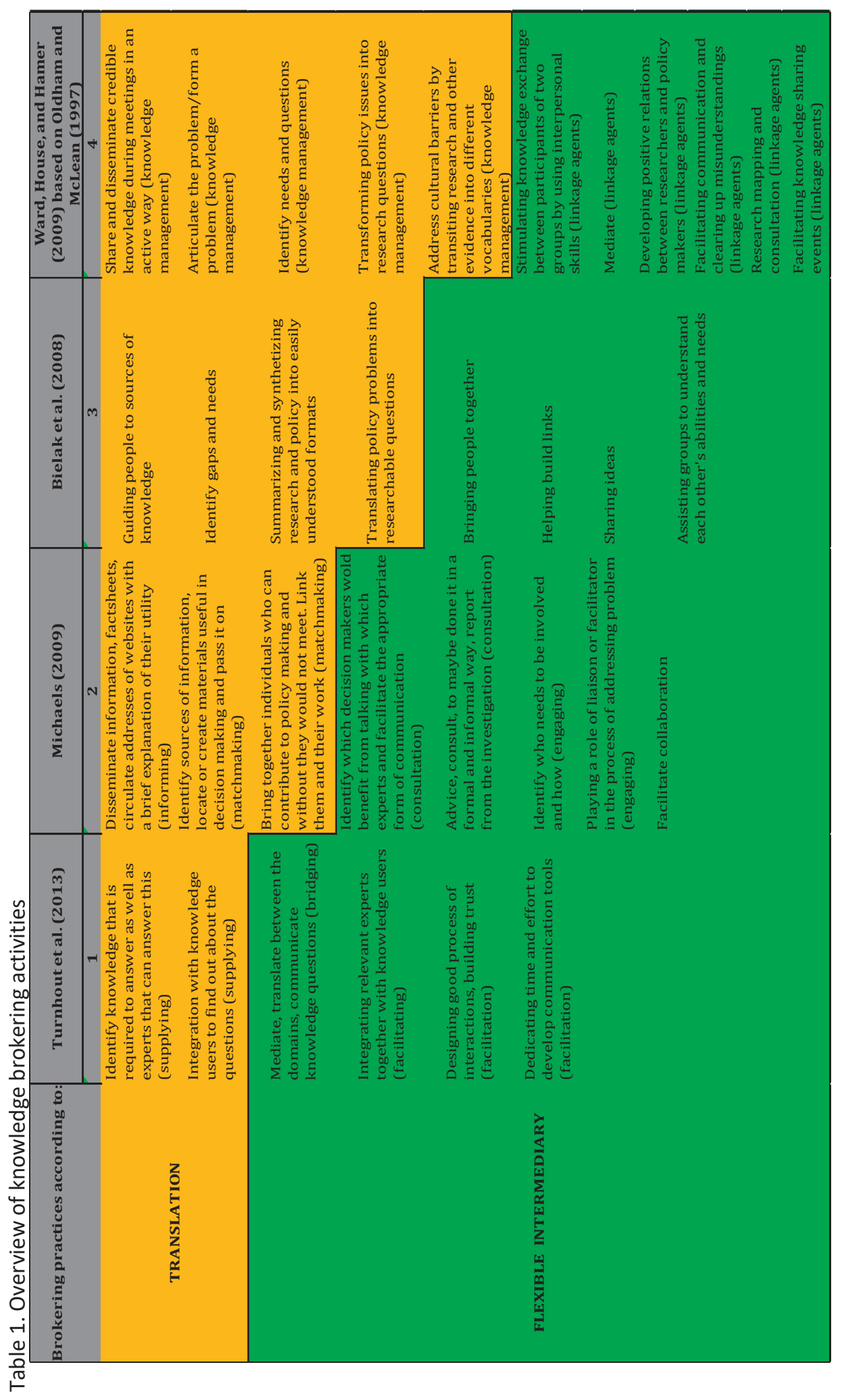




\subsection{Case description and methods}

This chapter uses the PP contract entitled Mainstreaming climate change into rural development policy post 2013 as a case study. In this contract, the public procurer was represented by policymakers of the Directorate General (DG) for Climate Action of the EC and the supplier was a consortium of six scientific institutes. In the PP contract a scientific study had been commissioned that aimed to deliver technical guidance and guidelines for managing authorities in member states on types of operations fitting to rural development measures that prioritise and address climate objectives. The contract lasted 9 months from December 2013 until September 2014. It included five tasks: task 1 identified 25 new and innovative climate actions to be described; task 2 examined how climate actions have been and could further be integrated into rural development measures in the EU; task 3 identified possible combinations of rural development measures that would increase synergies for climate objectives; task 4 identified criteria to measure the contribution of rural development measures and their combinations to climate change objectives and green growth; and task 5 focused on outreach and consultation with practitioners, dissemination of project results through a project workshop, and outreach tools.

We used this particular contract as a case because the EC is an important user of research and because it commonly uses PP procedures to purchase studies for delivering evidence to support institution's own policies or activities. This is related to the fact that the EC has recognized PP as a major component of the organisation of supply and demand of research in the EC research action plan (European Commission 2003). The EC has a well-developed internal PP procedures and requirements that can serve as good PP arrangements for production of scientific studies that are products of intellectual services and refers to the production of a scientific analysis of a de facto or de jure situation in the form of a written report (European Commission Vademecum 2013).

The procurement of a study involves the step-wise process including definition of the initial concept and identification of knowledge needs, acquisition by a third party (scientific or analytical entity), and production of the final report (Gershon 1999). Overall, PP contracts differentiate two phases: preparatory and execution. In the preparatory phase it is a role of a public procurer to define a policy problem, objectives and tasks of a study in an 
understandable manner to present it in a written form in tender documents. The execution phase refers to the process of knowledge production. In this phase, the experts perform their research and the public procurer monitors progress through communication and interaction with the experts.

We focused our analysis on how policymakers, the public procurers in our case, contributed to the creation of usable knowledge and the different knowledge brokering activities they used in the process of the PP contract. In particular, we analysed the knowledge brokering activities that have been used by the policymakers in the two phases of one PP contract: the preparatory phase and the execution phase.

Subsequently, we used our analysis to reflect on how PP provides conditions to build sciencepolicy relations. The analysis offers insights into the contribution of procurement procedures and knowledge brokering activities to the functioning of science-policy interfaces and the creation of usable knowledge. Altogether, the case study presented in this chapter offers a further exploration of the demand side of the science-policy interface and enhances understanding of the role of knowledge brokering activities in reconciling the supply and demand of knowledge (Sarewitz and Pielke 2007).

Our data collection strategy included literature and document study, participant observation and qualitative interviewing. We used eight in-depth interviews with persons: i) responsible for PP arrangements in general (policymakers of the EC); and ii) directly involved in this PP contract (policymakers of the EC or scientific experts from the supplier team) to ask on activities and processes that occurred in two phases of the PP contract and helped achieving understanding between the demand and supply side. None of the interviewees or other persons involved in the PP contract directly (on the procurer or supplier side side) were professionally trained to perform the role of knowledge broker. Participant observation was done by the first author who took part in majority of meetings during the execution of this PP contract. Participant observation was used to report on types of activities that reconciled the demand and supply side successfully. We coded our collected data for the occurrence of activities related to categories of 'translation' or 'flexible intermediary'. 


\subsection{Results: knowledge brokering activities}

This section presents knowledge brokering activities that were identified in the two phases of the PP contract.

\section{The preparatory phase}

The preparatory phase is an internal phase done by the policymakers of the EC only, therefore the brokering activities took place inside the organization. In our case study, one responsible policymaker of the DG for Climate Action was authorized to complete the preparatory phase and he acted as a knowledge broker by performing the following knowledge brokering activities:

\section{Articulating the problem and the policy need and consulting them with others}

The articulated problem was to identify possible actions to mitigate emissions and adapt to climate change in the agriculture sector that could be included in rural development policy. There was a strong belief amongst policymakers of the DG for Climate Action that rural development policy offered good opportunities for this and that the inclusion of innovative actions could make an effective contribution to climate change mitigation and adaptation.

The work of the knowledge broker focused on: i) identifying existing and credible sources of information and research, ii) synthetizing what had been done already in this field, and iii) planning what other knowledge were needed that could add to already existing knowledge. In addition, he organized internal consultations with policymakers of the DG for Agriculture and Rural Development (AGRI) (via face-to-face meetings and phone conversations). These activities provided sufficient basis for the planning of a contract (containing study report and communication activities), the content, and outputs. At the end of 2012, the first internal project proposal (a formal internal step) was prepared to ensure financial resources and to set the timeline for the final outputs.

Translating the problem and the policy need into more research-oriented tasks for the tender specification

The final outputs are specified in the tender specification. The EC has its own internal rules about the kind of information to be included in the tender specification of different types of 
contracts. According to these rules, the tender specification of a scientific study should include:

- background information about the research theme and its relevance for the EC;

- an explanation of the expected results of the research;

- a plan for the organization of the research, including the interaction between the EC and the supplier;

- a plan for communicating the results to the broader audience of potential users (optional).

The knowledge broker took care of the writing of the tender specification and discussed it in a small group of policymakers to make sure that the texts were clear. This tender specification was written in an open manner, meaning, for example, that the formulation of expected outputs was kept relatively open in order to give space to the supplier to develop his own ideas. It was requested in tender specification that the supplier develops technical guidelines. Whereas the necessary research steps to include, while developing the guidelines, were mentioned, the form of the guidelines was not specified purposively, so the supplier could come up with his own idea.

\section{Planning the interaction process}

The tender specification also required a planning: of interactions between the supplier and the EC, communication, and dissemination of the results. Therefore, the knowledge broker planned formal face-to-face meetings every time after the delivery of one of the outputs. Also, he planned the following outreach activities: i) to inform policymakers in member states about the importance of mainstreaming of climate change into rural development policy and by presenting them results of this PP contract, and ii) to get feedback from policymakers on what could be interesting and helpful while designing climate actions in rural development policy at national levels. 
Identifying experts to carry on the research

The tender specification explained what criteria would be used to award the winning tender (this also is a requirement of the PP standards) ${ }^{10}$. With regard to the identification of experts to carry out the research, it is not allowed to mention names of individuals or organisations. Nevertheless, the knowledge broker could set criteria about the required experience and level of education that experts should have and about the topics in which they should be competent (in this case rural development policy and climate change). These criteria ensured the involvement of credible experts with relevant knowledge.

\section{The execution phase}

One responsible policymaker of the DG for Climate Action was authorized to manage the execution phase and he acted as a knowledge broker. It was the same person that fulfilled the role of the knowledge broker in the preparatory phase. This was an advantage because he could clearly express the policy relevance of the contract. Part of his job related to purely administrative activities such as fulfilling the administrative and financial parts of the contract and launching a Steering Committee. The other part related to the management and facilitation of the interaction process between the procurer and the supplier. In this phase, the following knowledge brokering activities could be identified.

Bringing individuals together, identifying who needs to be involved and how

One of the knowledge broker's tasks was to constitute a Steering Committee.

There is always a Steering Committee in the EC's contracts that should be helpful to do the technical part of the work, so related to the content, the Committee is there to ensure the project's quality. (source: Interview with the EC's representative).

The knowledge broker searched for persons that would be able to provide good content guidance with regard to this contract. Therefore, he invited to the Steering Committee knowledgeable persons from the DG AGRI, other units of the DG for Climate Action, and a representative of a member state, so a potential user. They all took part in official face-toface meetings between the procurer and supplier and their role was to understand the

\footnotetext{
${ }^{10}$ In response to the written and published tender specification, potential suppliers proposed their offers. The European Commission in a selection procedure selected one supplier based on the evaluation criteria. Due to the confidentially issue we omit the selection procedure in further analysis.
} 
supplier's research methodology and provide comments to make the research results more relevant for policy work. At the later stage, the knowledge broker took the initiative to enlarge the Steering Committee to involve researchers from the EC Joint Research Centre, who were asked to provide feedback on methodology. Everybody in the Steering Committee had relevant knowledge to offer and the knowledge broker made sure that this knowledge was transferred to the supplier at the right moment. He coordinated comments coming from members of the Steering Committee to the particular deliverables; he consolidated them, and delivered to the supplier.

\section{Facilitating communication}

The knowledge broker facilitated communication and exchange of knowledge between the procurer and supplier throughout the whole time of running the execution phase, so during all meetings. Not only was the knowledge broker partly responsible for the organization of the meetings, he also participated in them. The meetings and communication strictly followed the plan included in the tender specification, almost to the exact days. Communication took place via channels such as face-to-face meetings, telephone, video conference, and additional direct calls that were not included in the communication plan.

The interactions between the procurer and supplier started very early in the execution phase. The first meeting took place a few weeks after the signing of the contract and after the delivery of the first output (it was an inception report). This first meeting was important as it allowed the procurer to explain its demands and the supplier to make sure that these demands of procurer had been understood and feasible. As an interview with a supplier's representative explains, this meeting was a key meeting in reconciling the supply and demand:

Being a supplier, I look on what the tender specification has asked for and what we as a supplier proposed to do in our offer. The first meeting is a key meeting in the communication process to clarify which parts are the most important to the procurer. So I would always go back to our offer to see what we said we are going to do and how this fits with the procurer's needs.

What was also important in the communication process was that the knowledge broker saw the advantage of introducing the whole supplier's team (so all researchers involved) to the procurer. On the side of the supplier there were three different research groups distributed over different countries, and they did not intend to participate in all face-to-face meetings (mainly due to saving costs of traveling). So, in order to meet/see them all, the second 
face-to-face meeting was changed into a video conference which helped to create a proper linkage between the procurer and supplier without bringing everybody physically to the same room.

I think that it is very important to communicate directly with as many people on the supplier team as possible and be able to listen to their questions of what they understand and what they intend to deliver. In this way we can clarify very quickly and directly any potential misunderstandings. And persons who are really going to do the research on the supplier side are able to see that their research contribution is being understood and appreciated. (source: Interview with the EC's representative).

The knowledge broker was available for the supplier whenever additional informal communication was needed (additional calls, video conferences) to discuss issues with the execution of tasks or to inform the supplier about whether he was satisfied with the research progress and particular outputs.

Developing positive relationships and mutual understanding

The knowledge broker put effort into developing the relationship between the procurer and supplier. He ensured that everybody who was present on meetings/video conferences/calls had the opportunity to make comments, give input and be understood:

In this kind of relationship, it is important to ensure that everything that the public procurer is trying to explain is clear and understood in both directions. And that there are reactions to any problem that the supplier raises. (source: Interview with the EC's representative).

One example related to this appeared while discussing task 4. During the first meeting, one member of the Steering Committee explained his expectations towards this task. Specifically, he expected that the research would deliver a full matrix of indicators for evaluation of green growth contribution. This would have been very costly and time consuming and did not fit in the budget and time planning for this task. Consequently, the knowledge broker stepped in and limited this task to what was reasonable and possible. He also gave a clear message about what priorities were important for his unit. This example illustrates that the knowledge broker also played an important role in mediating between the supplier and the Steering Committee.

Another example was that the knowledge broker used visualization to ensure that his demands were well understood by the supplier. In order to execute task 1 (to prepare 25 new and innovative climate actions and describe them), a template was provided in the tender specification, which was later on discussed with the supplier during one of the meetings. This 
template served well in clarifying and communication the expectations of the procurer and helped the supplier to describe the climate actions accordingly.

\section{Being engaged and bringing inspiration}

The knowledge broker devoted time to read all outputs that were in a form of reports carefully and to comment on them. By presenting comments in a form of open questions he intended to inspire the supplier, as explained below:

Although sometimes I also have a big knowledge of the subject, I know what is relevant and have ideas how to do things, I should not forget that the contract is to find the answers/solutions by some experts on the supplier side within the certain time. There is no need to do work for the supplier. [...] The management of the content is to trigger the experts to change their thinking, to challenge them so as they innovate, and give them space to do their work. (Interview with the EC's representative).

This demonstrates that the role of the knowledge broker was directed towards inviting the supplier to create new proposals rather than to the generation of new knowledge himself. The communication process was used to make sure that the new proposals would not be too far from the procurer's demands.

Guiding to sources of knowledge and understanding research possibilities and limitations

The knowledge broker provided the supplier with credible sources for data by linking the supplier's team to other EC representatives. He also looked for other sources when the supplier raised a problem with finding data.

One example from the case study illustrates this. The supplier asked for data from the FADN ${ }^{11}$ (Farm Accountancy Data Network) so the knowledge broker made an availability request to colleagues in the DG AGRI who owns and manages this database. Having this data would have definitely added to the analysis and it would have made the process of selection of the most climate relevant measures in task 1 more robust - said one respondent on the supplier side. But the data set was not available on time and what had been received later on was fragmented and dispersed so it was not possible to use it in the analysis. When the supplier raised this issue to the knowledge broker, a new strategy for the selection of actions was discussed. The knowledge broker identified another group of experts that could be helpful in

\footnotetext{
${ }^{11}$ The FADN were needed to identify types of climate change actions to which allocation of rural development payments at the farm level can be traced.
} 
selecting actions; these were experts from EC Joint Research Centre and subsequently, they were involved in the project within the Steering Committee.

Several of the interviews pointed out that this procurement contract was very complex methodologically. As one respondent stated:

The contract required a lot of data collection and processing, and also methodologically it was not easy. for example how do we do a robust list of actions in task 1. There were so many variables involved to assess which actions are the most relevant so at the end we also needed to use the expert judgment. (Source: interview with a supplier's representative).

The knowledge broker was able to steer the supplier whenever problems regarding research appeared and had been communicated.

The knowledge broker understood what can realistically be delivered within the contract, he was able to prioritize what is the most important for the EC when it comes to relevance, he was clear about it, he was able to indicate things that were less important. He also understood that when we were struggling with some data and the limits of days we were assigned for this contract, he was able to indicate on which parts we should focus, where the priorities are, he was steering us, and guided us towards his, so the Commission, point of view. (Source: interview with a supplier's representative).

\section{Facilitating external communication: workshop}

As one EC respondent explained, an external workshop is often a part of the tender specification of the EC. For this contract, it was the main activity that targeted potential users, that is, policymakers in member states. The knowledge broker ensured that the workshop was well planned, that its goal was achieved and the outcomes of the workshop were used while forming final results. He discussed the workshop plan and a list of potential participants with the supplier during one of the face-to-face meetings. It was agreed that the workshop would consist of a combination of presentations and group discussions and that it would focus only on those topics that were the most important to the EC.

Around 40 users took part in the workshop, mainly policymakers from member states, but also experts representing the DG AGRI, Birdlife, Copa-Cogeca. The workshop served not only to communicate research results, but also to raise awareness of the possibilities to include climate change actions into rural development policy. All our interviewees confirmed that the workshop was useful:

It was valuable that he gets feedback from the potential users during this workshop because the supplier worked on something new. (Source: Interview with the EC's representative). 
During workshop we received some practical comments (as researchers we knew less about the practicalities of implementation and policymaking). We let people commenting to make sure that the way we presented our outputs was understandable, comprehensive, and relevant for them. At the same time, we raised awareness about climate relevant operations among policymakers. (Source: Interview with the supplier's representative).

Interviewees made clear that workshop enhanced the usability of the outputs. It provided an opportunity to get users involved and gave them space to provide practical comments, for example regarding the potential implementation of the selected actions.

The workshop was very useful because it prevented the researchers from developing a bit of fantasyit helped them to put something in place because they do not understand the practicalities that the administration faces. And it also delivered to the administration some new ideas. (Source: Interview with the EC's representative).

So, workshops are fundamental part of the communication process in some contracts. If we just produce reports and pamphlets and hand them out, these contracts do not have much impact but when people come to workshops to give us feedback on these reports and pamphlets, they do put a lot of value in it. (Source: Interview with the EC's representative).

Summarizing, the workshop was helpful for the supplier in learning about the demands of users and incorporating them into their work.

\subsection{Discussion}

Our case study exemplified the role of a knowledge broker in a PP contract. It offered an example of how actors on the demand side of the science-policy interface (such as policymakers) can contribute to the creation of usable knowledge and to building productive science-policy relations. The choice of knowledge brokering activities by the policymaker was specific for this PP contract and is not easily transferable to other contexts. This is not only because knowledge brokering activities were tailored to specific policy problems, but also because these choices were influenced by the knowledge broker and the ways he perceived and performed his role in reconciling the supply and demand of knowledge (see also in Saarela et al. 2015).

Our analysis has demonstrated two knowledge brokering activities related to 'translation' in the preparatory phase of the PP. The preparatory phase concentrated on the internal process at the side of the procurer of identifying needs and articulating the research problem. These translation activities included not only working with information (processing scientific information that was produced earlier and including it in the tender specification) but also 
working with people (internal consultations amongst policymakers on policy needs). Specifically, policymakers engaged in translation to explore the aims and scope of the study and to communicate these to potential suppliers. Other knowledge brokering activities related to 'flexible intermediary'. These included the planning of the process and the management of the interactions between the different people involved. The planning of these interactions was a substantial step and highlighted the importance of dialogue between the demand and supply of knowledge. Also in the execution phase, knowledge brokering activities helped in the management and facilitation of interactions between the demand and supply sides.

Understanding how to align knowledge supply and demand was an important and constant element within this PP contract and the policymaker of the EC acted as an effective knowledge broker by carefully tailoring the activities to ensure this alignment (Sarewitz and Pielke 2007). Our case study showed how the knowledge broker within this PP arrangement used knowledge brokering activities to clearly describe what the research need was and to guide the suppliers so that they would meet this need and provide useful results. The fact that knowledge brokering activities were taking place continuously was very important for the process of building relations between the policymakers and researchers. A variety of communication tools were applied including external communication with potential users - policymakers in member states who dealt with rural development programming. Frequent communication brought higher levels of trust and understanding of each other perspectives, which are crucial for successful science policy relations (Saarela et al. 2015; Gaudreau and Saner 2014).

Most documented examples of knowledge brokering focus on experts, either outside specialists/consultants or members of the supplier team as knowledge brokers (Saarela et al. 2015; Holzmann 2013). It seems that such experts have the resources and capacity to create these kinds of roles within their organisations and may have more perceived legitimacy in guiding their colleagues towards work in areas that are likely to be more useful to end-users (Bielak et al. 2008). In our case, it was a policymaker who played the role of knowledge broker. Valente et al. (2014) have pointed out that the quality of scientific advice depends on the clarity and quality of the knowledge user in being able to identify and communicate what his needs and requirements are. So, the demand side is considered to have the main responsibility for the quality of the advice. Our case study confirms that indeed policymakers can make 
a positive contribution to the advice process and can even play the role of knowledge broker. To some extent, this was the result of some specific circumstances where in our case the policymaker had relevant scientific expertise and skills and was therefore ideally suited for this role. However, this will not always be easy to arrange and it might be a good idea to specifically train policymakers who work on daily basis with research PP contracts to play this role. We see some of this being taken up in the private sector where workers are being trained to become knowledge brokers (Ishiyama 2016), and also in policy teams where specific civil servants are tasked to improve science-policy dialogue (Bielak et al. 2008).

\subsection{Conclusions}

By demonstrating the importance of continuous interaction and by highlighting the important role of knowledge brokering activities, our analysis contributes to our understanding of how to ensure productive relations between science and policy that are able to avoid the pitfalls and shortcomings of the linear science-policy model. (Beck 2011; Wesselink et al. 2013). We recognize that since our analysis is based on a single case study, caution is warranted in such general claims. Indeed, the existence of a PP framework is not a guarantee that every PP contract can be executed smoothly and without major difficulties. Further empirical testing will be important to investigate the functioning of PP contracts, for example in other governmental settings outside the EC. Nevertheless, our study does suggest that PP could serve as a model to improve science-policy interface also in other contexts. What is specific about PP that could be considered in these other contexts is that it offers a governance framework for science-policy interfaces (see also Van Kammen, De Savigny, and Sewankambo 2006; Carter 2013; Görg et al. 2016). Specifically, PP offers a framework with clear rules and with a clear role of the demand side in governing the knowledge production process. This is relevant for science programming more generally. Whereas currently, the interactions between knowledge users and producers are often voluntarily, programmed in the final work package, or allocated limited budget, clearer rules and governance frameworks may result in science-policy interface related matters being taken more seriously also by policymakers form the start with better outcomes for the usability of research. 


\subsection{List of interviews}

List of interviews:

Eight non-standardized interviews with the representative of the European Commission and the representatives of the contractor's team took place. Their duration lasted from 40 minutes to 2,5 hours. The list of interviewees is presented below:

- Representative of DG Budge to ask for the general tender procedures (June 2013).

- Representative of the DG SRD to ask for the specific rules for contracts of research/study for the DG for Climate Action (June 2013).

- Representative of the DG for Climate Action who was involved in the tender preparation and later managed the project (first interview in June 2013, second interview in September 2015).

- Representative of the DG for Climate Action not involved in this project to learn more about the general procedure for tendering in this DG for contracting research (June 2013).

- Representative of the DG for Climate Action not involved in this project to learn more about the general procedure for tendering in this DG and contracting research (July 2013).

- Project leader of the side of the supplier (August 2015).

- Project team member of the side of the supplier (December 2015).

Participant observation:

I have participated in almost in all meetings during the time of executing the investigated public procurement. The meetings embraced formal meetings of the communication plan as well as additional informal meetings. Notes have been done during the following meetings:

- Kick off meeting - Video Conference, 18 Dec 2013.

- Inception meeting in Brussels, 31 January 2014.

- Interim meeting - Video Conference, 27 March 2014.

- Teleconference with presenting the draft results, 16 April 2014. 
- Presence on the meeting when results have been presented to the policymakers from member states on the occasion of some other meeting in Brussels, 23 May 2014.

- Workshop with the policymakers from member states to test the first results of the project, 17 June 2014. 
CHAPTER 5

\section{HOW POLICYMAKERS' DEMANDS FOR USABLE KNOWLEDGE SHAPE SCIENCE-POLICY RELATIONS IN ENVIRONMENTAL POLICY IN POLAND}

This chapter has been published as:

Kowalczewska, K., and Behagel, J. (2018). How policymakers' demands for usable knowledge shape science-policy relations in environmental policy in Poland. Science and Public Policy. https://doi.org/10.1093/scipol/scy065 


\section{Abstract}

This chapter discusses how aspects related to policymakers' demands for knowledge shape preferences for science-policy models such as Mode 1 and Mode 2. It focuses on the demands that Polish policymakers make of science and how they envision their role in the knowledge production process in the field of environmental policy. The chapter applies a set of criteria on how policymakers define usable knowledge to better understand preference and use in practice of different science-policy models. Results show that preferences for Mode 1 or Mode 2 are in part the result of trade-off between criteria of quality, relevance, conformity, and action orientation. While science can provide truth and usable knowledge in both Mode 1 and 2, Mode 1 is attractive when policymakers have specific political demands: they may use it to avoid responsibility for negative policy outcomes or to discredit undesirable results.

Key words: Mode 1; Mode 2; Poland; science-policy models; usable knowledge 


\subsection{Introduction}

Scientists are important providers of expertise: one can say that academia and society have come to an agreement that science is the best knowledge we have of how the world works (Yearley 2004). Accordingly, scientific knowledge is meant to be used and applied in policymaking. Yet, how scientific knowledge moves from production to use is a question without a clear answer. Over the past 50 years, one can witness an intense and evolving debate on how science, policy, and society relations are and should be structured (Kirchhoff et al. 2013). This debate moves from a discussion of the merits and limitations of the linear and mono-disciplinary model (Bush 1945) to the consideration of more complex, interactive, and interdisciplinary models of science production that include user involvement and sensitivity to societal problems (Gibbons 2000; Nowotny et al. 2001; Landry et al. 2003; Jacob 2006; Kirchhoff et al. 2013). The debate emphasizes different aspects of changing relations between science, policy, and society and seeks to understand efforts to enhance the quality, effectiveness, and legitimacy of knowledge and expertise in policymaking (Turnhout et al. 2013).

An influential description of the changing relations between science, policy, and society has been offered by Gibbons et al. (1994) and Nowotny, Scott, and Gibbons (2003), in the form of the Mode 1 and Mode 2 discussion. They use the term Mode 1 to denote the conventional model of knowledge production within scientific disciplines. In this model, science-policy relations are linear and "problems are set and solved in a context governed by the (largely academic) interests of a specific community" (Kraak 2000, p. 35). Mode 1 is characterized by knowledge production that draws on homogeneity of skills and hierarchical organization of the scientific endeavour. In contrast, in Mode 2, knowledge is produced in a context of application and can involve a much broader range of social perspectives. Mode 2 is thus based on inter- or transdisciplinary, heterogeneity, and organizational diversity. Moreover, Mode 2 shows a preference for flatter organizational structures which are transient (Kraak 2000). In both models, peer review serves as quality control, but in Mode 2 it involves a more temporary and heterogeneous set of participants who are interactively involved in discussions on contextualized problems. As a result, Mode 2 is thought to be more socially accountable and reflexive than Mode 1 (see also Gibbons et al. 1994, p. 3-8, 167; Nowotny, Scott, and Gibbons 2003). 
We consider the Mode 1/Mode 2 discussion to be embedded in multidirectional changes in thinking about knowledge production and use: from mono to transdisciplinary (also captured by a concept of transdisciplinary research by Klein et al. 2001; Regeer and Bunders 2009); from linear to multidirectional and social processes of knowledge co-production (Jasanoff 2000); from expert-based to democratic knowledge production (also captured by Funtowicz and Ravetz 1993 in a concept of post-normal science); and from an emphasis on knowledge production to an emphasis on knowledge use, application, and policy relevance (Mode 2 of Gibbons et al. 1994). It is important to emphasize that scholars like Gibbons (1998) have not argued that Mode 2 and other models (transdisciplinary, post normal science, and co-production) are going to eliminate the old paradigm of Mode 1. Even so, the societal trend is towards more complex and interactive models of knowledge production that include users (Metze and Turnhout 2014).

Notwithstanding the above, Mode 1 remains in use due to various reasons (Turnhout, Neves, and De Lijster 2014). Scientists who distance them- selves from policy find it easier to maintain credibility and authority (Wooster 1998). They do so by drawing on the assumption that knowledge produced in Mode 1 is independent, objective, and free of influences (an assumption still embraced by many). Mode 1 is also still supported by many governmental institutions and policy- makers (Kraak 2000). For example, Wesselink et al. (2013) write how global climate change governance is built on a linear approach to science-policy interactions. Moreover, knowledge produced in Mode 1 is perceived by policymakers to be isolated from political considerations and thus useful for policymakers to distance them- selves from policy decisions and avoid public responsibility (Gieryn 1983; Flinders and Buller 2006). However, many policymakers acknowledge that knowledge produced within Mode 2 often offers better solutions to societal problems and can deliver relevant, legitimate, and credible knowledge (as defined by Cash et al. 2002; 2003). In Mode 2, there is also more awareness that knowledge is value laden.

We hypothesize that the demands that policymakers make on knowledge-that in turn affect their preference for either Mode 1 or Mode 2-will be shaped by whether they intent to use this knowledge either politically or instrumentally. Accordingly, such demands may lead to preferences for the Mode 1 or Mode 2 model depending on the situation. How to link policy demands to preferences for Mode 1 or Mode 2 is not immediately clear. Kunseler and Tuinstra 
(2017) found that experts seek to purvey objectivity and authority, while navigating different models in their practices. Weiss (1979) already found in early studies on knowledge use that policymakers expect science to deliver empirical, objective evidence and conclusions that in principle help to solve a policy problem-a typical instrumental use of knowledge. Wesselink et al. (2013) found that when demands for knowledge are linked with political sensitive contexts of use, knowledge becomes ammunition for the side that finds its results supportive and it becomes congenial when it cannot be undermined. In other words, policymakers may benefit from scientific knowledge that is isolated from interactions with policy and therefore give preference to Mode 1, but they may equally benefit from knowledge that is co-produced with users and for a specific context of application and thus give preference to Mode 2. In addition, policymakers may have a preference for one mode or the other based on broader policy contexts.

This chapter explores how models such as Mode 1 or Mode 2 may satisfy the demands that policymakers make of knowledge production and use. We use the Mode 1/Mode 2 terminology as an umbrella term to discuss policymakers' preferences for either monodisciplinary and linear models or more complex and interactive models of science, policy, and society relations. To inform analysis, this chapter draws on the usability criteria of Weiss (1995) to identify different policymakers' demands on knowledge and how these relate to the Mode 1/Mode 2 debate. Accordingly, we discuss the influence of policymakers' demands and preferences on practices of knowledge production and use, a topic less explored in literature (Bielak et al. 2008; Dunn and Laing 2017). Our results are based on an analysis of environmental policy in Poland, where we found both Mode 1 and Mode 2 models (Kowalczewska, Behagel, and Turnhout 2017) to be relevant.

\subsection{Criteria for usable knowledge}

Defining criteria for usable knowledge is an important topic in knowledge utilization studies and is emphasized in the literature in relation to many sectors (environment, health care, and education). Scholars have come up with partly overlapping and different sets of criteria of what usable knowledge is (Rich 1991; Weiss 1995; Dunn and Laing 2017) and have also indicated trade-offs between criteria (Cash et al. 2002; 2003). Weiss (1995) and colleagues carried out a research amongst decision makers, focusing on the community of policymakers 
in the USA and a broad field of policy subjects, to value the usefulness of actual studies for their own work. This research yielded five main criteria important for usable knowledge: (1) relevance, (2) conformity, (3) quality, (4) action orientation, and (5) challenging the status quo. Although these criteria have been developed more than 20 years ago, they bring together the dimensions of knowledge production and use, both of which are relevant to the sciencepolicy interface (Tangney 2017), especially in Mode 2, where they are considered strongly entwined.

Policymakers' demands on knowledge may place different emphasis on different criteria depending on the intention of use (political or instrumental) as well as the general policy context in which they are situated. These criteria may be satisfied by science-policy relations that may be found either in Mode 1, Mode 2, or both at the same time (Gibbons 1998; Kraak 2000). A recent study by Dunn and Laing (2017) shows that users such as policymakers under certain conditions do not necessarily desire knowledge to be legitimate and credible as much as they prefer it to be policy-relevant. They, therefore, propose that research should consider 'applicability, comprehensiveness, timing, and accessibility' (ACTA) (Dunn and Laing 2017) rather than 'credibility, relevance, and legitimacy' (CRELE). The latter set of criteria have been made popular by Cash et al. (2002) and have indeed been rapidly adopted by the scientific community to serve as a standard by which to evaluate knowledge production and use. Both sets of criteria can be used "to better understand competing demands at the science-policy interface" (Tangney 2017, p. 149). Weiss (1995) and others, already in the 1980s and 1990s, have developed five criteria that includes elements of both ACTA and CRELE. Therefore, we use them as 'heuristic tools' to study how models such as Mode 1 or Mode 2 can produce knowledge of particular characteristics that do or do not satisfy policymakers' demands on knowledge. Below we present descriptions of each of the five criteria as defined by Weiss (1995). In our descriptions of the criteria, we include insights from recent literature on knowledge criteria. Crucially, we provide information for each criterion how Mode 1 or Mode 2 science-policy relations can or cannot satisfy the demands that are related to it.

The first criterion is relevance. Relevance is considered a key attribute of science-policy interfaces by a broad range of authors (Cash et al. 2003; Farrell and Jäger 2006; Kunseler et al. 2015; Dunn and Laing 2017). Knowledge is usable to policymakers when it is relevant to the policymakers' own work (Weiss 1995), which means it is delivered timely and relates to topics 
that are societally relevant and match with policy and societal needs (Sarkki et al. 2015). To increase utility by policymakers, scientists should thus produce time-critical research rapidly enough to affect policy outcomes (Laurance et al. 2012). In Mode 1, such timely and societal aspects of relevance are often lacking. In the field of conservation science, for example, many studies do not links with real-world conservation outcomes (Knight et al. 2008; Milner-Gulland et al. 2010). Laurance et al. (2012) suggest that this is because the majority of conservation scientists are in academic positions and have limited interactions with conservation practitioners and managers (see also Campbell 2007; Milner-Gulland et al. 2010). While in Mode 1 scientists do research on matters they perceive as urgent and problematic, they do not necessarily follow policy agendas and many studies stay unused (Kirchhoff et al. 2013). According to Laurance et al. (2012), this calls for communication and dialogue between conservation scientists and policymakers and practitioners. This view is shared broadly across policy domains: many studies emphasize that the involvement and participation of users (practitioners) in interactive knowledge production processes (i.e., Mode 2) leads to the production of relevant knowledge for policymakers and improves the level of its usage (Landry et al. 2003; Lemos and Morehouse 2005; Jacob 2006; Chapman et al. 2015).

The second criterion is conformity to the prior knowledge, experience, and belief of policymakers (Weiss 1995). Conformity can be achieved through a process of knowledge translation. For example, knowledge translation targeting policymakers should ensure that consideration of research evidence is a key component of decision making, but also recognize that there are other legitimate factors that need to be considered (Grimshaw et al. 2012). Knowledge translation processes thus ensure that policymakers are informed about research results. Knowledge translation in Mode 1 can be done by scientists who translate results into more readable formats, but their translation abilities and understanding of policy language and context is often limited. As Shonkoff and Bales wrote (2011, p. 30):

the challenge of translation can be addressed within a mutually respectful, ongoing collaborative process in which developmental scientists, communications researchers, and policymakers can become co-producers of broadly understood yet sophisticated scientific messages that are not 'dumbed down' yet take into account the cognitive shortcuts that non-scientists bring to the discussion of complex issues.

Thus, the argument is that following a Mode 2 model allows scientists to present knowledge that fits the policymakers' specific needs and combines it successfully with their knowledge 
base (Den Hertog 2002). To support knowledge translation processes and simplify messages for policy it is moreover emphasized (e.g., in Sarkki et al. 2013) that policy briefs, pictures, maps, and figures may be efficient translation tools for policymakers.

The third criterion is quality. Useful knowledge should be of high quality, which includes references to scientific standards related to methodology (Weiss 1995). Quality of knowledge has also been discussed in term of credibility (Cash et al. 2002; Farrell and Jäger 2006), which refers to the (perceived) quality, validity, and scientific adequacy of the knowledge exchanged at the interface. It includes credibility both of the knowledge production processes and of the knowledge holders (in Sarkki et al. 2013). In Mode 1, quality research can be achieved through very strict methodology that is used to discover facts and is carried out mainly at universities (Kirchhoff et al. 2013). Science then can be perceived as objective, free from emotions, private interests, bias, or prejudice. This means that scientific knowledge is acquired through systematic experimentation with nature and results can be reproduced to check if they are true or not (Kirchhoff et al. 2013). In Mode 2, quality is more a matter of peer review. One the one hand, Mode 2 science is flexible enough to produce for example multidisciplinary research by involving scientists from different disciplines into the production process and to involve other types of (non-scientific) knowledge holders in processes of co-production (Jasanoff 2000). Many studies emphasize the importance of multidisciplinary research projects because they "build on theories and previous research from more than one discipline and use methods for data collection and analysis from more than one research tradition" (Locker 1994, p. 138 cited in Sumner 2003, p. 2) and lead to more 'socially robust' knowledge (Hegger et al. 2012). However, some knowledge holders may either politicize or technicize expertise (Wesselink et al. 2013) to attach it to political values or actively isolate it from them. Both politicizing and technicizing weaken the credibility of co-production, as criteria of quality within respectively Mode 1 and Mode 2 become undermined. Equally, the credibility and objectivity of science produced may be weakened when scientists become too actively engaged in the policymaking process (Mills and Clark 2001; Wooster 1998).

The fourth criterion is an orientation to action. Weiss (1995) stated that decision makers prefer a study that gives them direction for tangible action. Action-oriented research generates knowledge that can be used to address practical concerns of local communities, organizations, and groups, to incorporate local understandings of specific issues (Small and 
Uttal 2005), and is often small-scale (Burns 2000). Within specific themes, for example adaptation to climate change, a call for action-oriented research is linked to a need for policy interventions to change behaviours across multiple sectors, requiring policy processes to reshape institutional settings (Lahsen et al. 2010). This may be part of Mode 1, as generalized results (e.g., global warming) call for broad policy action (e.g., climate mitigation action). However, action-oriented research is more often associated with Mode 2: it may produce information based on for example case studies that is really focus on the context of application (Small and Uttal 2005). Within all action-oriented research approaches, it is common that it involves some type of collaboration between researchers and users or policymakers (Small and Uttal 2005). How this collaboration takes shape depends on policy demands: more politicized demands will involve global orientation to action and Mode 1 type of quality, whereas policy demands focused on problem-solving may more readily lead to Mode 2 models.

The fifth criterion is for knowledge to be able challenge the status quo or the current policy agenda (Weiss 1995). Scientific studies can challenge current policies; especially the independent quality predominantly ascribed to Mode 1 science is associated with this criterion. Knowledge-driven studies (produced in Mode 1 organization) can thus be critical of current policy and offer different policy alternatives. Studies produced in Mode 2 can also bring fresh ideas into a specific problem, but more as a result from processes of co- production than from independent expertise. Thus, including multiple disciplines and types of knowledge may challenge status quo of policymaking, especially is this status quo had previously been based on Mode 1 types of science. Again, environmental science is illustrative here, as debates on science advocacy within this field move be- tween those that find it is the role of scientist to speak out to policy (e.g., 'truth to power') (Wildavsky 1987, also compare Al Gore's latest movie) and those that urge more transparency (Garrard et al. 2016) about normative positions and reflection on the relationship between researcher and policy communities (Small and Uttal 2005).

The above review shows that the first two criteria, relevance and conformity of knowledge, cannot be produced well in Mode 1 . The emphasis on these criteria is commonly associated by policymakers with the instrumental use and the literature suggests that this will steer policymakers' preferences towards Mode 2. Review of the quality, action-orientation, and 
challenging criteria of knowledge paints more complicated picture. Policymakers who have political demands for knowledge often choose Mode 1 because it considers science as independent from policymaking (part of the quality criteria). Mode 1 is also a more likely preference when policymakers' demands are in line with more global and generalized calls to action and when they consider it the task of scientists to challenge the status quo. When more instrumental demands dominate, demands for quality may also be satisfied in Mode 2 . Moreover, literature suggests that demands that focus on solutions for concrete localized issues, and policy change that originates from a broad range of stakeholders is also more likely to steer preferences towards Mode 2. Scholars such as Pielke (2007) show that in an interactive mode like Mode 2, science can also be considered independent if the focus of knowledge production and use is on opening up debate rather than closing it down.

In sum, policymakers will value different criteria for usable knowledge based on their policy needs and demands and will also interpret these criteria differently depending on whether their needs are more focused on allocating political responsibility or on being instrumental to achieving policy objectives. Moreover, trade-offs between criteria are likely, for example when demands for quality compete with the need for relevant knowledge. As a result, a combination of policy demands may lead to a preference for either a Mode 1 or a Mode 2 type of science-policy relations. Below, we flesh out this argument with an empirical case study of the knowledge demands of Polish policymakers and the models of science- policy relations that were in use.

\subsection{Case study approach and methods}

The empirical case relates to Poland that accessed the EU in 2004. The accession process required many laws to be changed in order to comply with the EU rules. But, complying with the EU rules is a continuous process that refers to all policy sectors and requires production of knowledge for policy convergence with the EU. For policy convergence, knowledge and expertise played an important role, in agriculture and environmental policies in particular (e.g., rural development and nitrates policy), by providing policymakers with data sets, indicators, interpretations, calculations, and new ideas to create policy solutions (Kowalczewska, Behagel, and Turnhout 2017). For these particular policies-rural development and nitrates policy-science-policy relations were dynamically influenced in the 
period between 2004 and 2016 by two factors. Firstly, a great amount of knowledge and expertise was required and produced within these policy initiatives. Secondly, these policies were subjected to political dynamics (Kowalczewska and Turnhout 2012; Kowalczewska, Behagel, and Turnhout 2017).

We adopted a qualitative approach to data collection. Our data derives from fifteen semistructured and face to face interviews with Polish policymakers working in the field of agriculture and agri-environment, carried out by the first author. Interviewees were selected based on the prominence of their participation in the implementation processes of rural development policy or Nitrates Directive and their institutional roles. During the interviews, interviewees were asked about: (1) use of knowledge in policy documents and developments, rural development, and the Nitrates Directive in particular; (2) types of knowledge delivered by scientists; (3) quality of delivered knowledge and other criteria important for knowledge to be used in policy; and (4) relation and interactions between policy and science.

As an analytical method we carried out a discursive analysis (Hajer and Versteeg 2005) to systematically study transcripts of interviews. In the analysis, we searched our data for policy discourses that were supporting Mode 1 or Mode 2, to identify preferences for one or the other within the general policy context in which interviewees were situated. In addition, we coded for the framing of policy demands according to the criteria of knowledge described above, including whether demands could be identified as political or instrumental. We used all the data provided in transcripts and coded with support of the QDA Miner Lite software. The next section describes our findings in detail.

\subsection{Knowledge demands of Polish policymakers}

\section{Relevance}

Polish policymakers defined relevant knowledge as being in line with European law and stated that relevant knowledge is more likely to be used. Experiences with implementing EU law in the first years after accession (e.g., agricultural policy) showed that scientists were not always fully aware/informed about the EU rules that have to be implemented in policy. Policymakers had an expectation, for example, that when programming the Rural Development Programme for 2007-2013, scientists would deliver the data and interpretation of the state of play that 
could be used directly to design rural development measures. They had been sending specific questions to scientists (according to the policymakers well formulated) but received answers that did not always fit their expectations and therefore could not be used. Similarly, when scientists were asked by policymakers to consult the programmed rural development measures sometimes their comments were not usable because they were not fully complying with EU regulations. Such science-policy communication failures are typical for Mode 1.

Relevant knowledge, for Polish policymakers, was also related to 'fit' and good timing in policy. They stated that information presented according to international standards is more likely to be used. This is important when presenting values of environmental indicators because then it is possible to compare them with the European average or other countries. Good timing was an issue within the implementation of the Nitrates Directive in 2007: there was a problem with synchronization of national implementation guidelines with the EU guidelines. First, the national implementation guidelines were used by regional water authorities (responsible for the Nitrates Directive implementation) to order scientific expertise (via individual legal contracts) with indicators showing levels of nitrates pollutions in waters. Later on, the European Commission sent the European guidelines for all member states and it turned out they differed from the national ones. Scientific expertise that was already acquired by policymakers had to be adapted in order to be used, or changed completely. So, timing was an important issue.

Another example highlighting the relevance of knowledge related to preparing a National Strategic Plan for rural development for 2007-2013 ${ }^{12}$ for which it was necessary to have a diagnosis of the state of agriculture in Poland. For this diagnosis, statistical data were used although these data did not always meet the requirements of this document. Therefore, additional information was provided by two scientific institutes subordinate to the Ministry of Agriculture. There was already a group of scientists who worked on this document, so the exchange of information between them and policymakers was interactive and effective (similar to Mode 2). Scientists developed and validated the descriptive and indicative parts so

\footnotetext{
12 National Strategic Plan 2007-13: this is an official document prepared according to the Council Regulation 1698/2005 on support for rural development. This document embraces the rural development perspective of 2007-13 and was necessary to be prepared and submitted to the European Commission for acceptance. Only after approval of this document, the Rural Development Programme 2007-13 for Poland could be submitted to the European Commission.
} 
that all strategic goals and priorities described in the document would reflect the current state of agriculture, according to the guideline for the National Strategic Plan. As a result, the delivered scientific information was relevant and could be directly used by policymakers.

\section{Conformity}

We wrote that conformity to the knowledge of policymakers requires the process of translation. Polish policymakers mentioned that they sometimes had difficulties with understanding scientific reports due to their written language which was named by respondents as 'very scientific' or 'too scientific'. Scientists had their own way of presenting scientific standards, regulated by the world of science. Monographs (single long manuscripts) can be painstaking to read for policymakers, said one policymaker. Condensation of results in a shorter document is more desired although this may cause a risk of misinterpretation of the results, according to another policymaker. In the case of Poland, the process of translating research results for policymakers was not mentioned as a common practice which would suggest that a traditional way of knowledge production, like in Mode 1, dominated.

Polish policymakers were fully aware that certain characteristics like visualization and good layout-so more user-friendly elements-are helpful in understanding scientific knowledge. Graphical representations and formats can also positively influence usability, in their views. For example, the way the EUruralis scenario study presented its results (historic and temporary facts, figures and pictures of the EU 27) (Kowalczewska and Turnhout 2012), by using maps, graphs, tables and four contrasting scenarios contributed to usability. Polish respondents appreciated this way of translating the results because it makes it much more attractive than a traditional scientific publication. They emphasized "the easiness of getting the results of prognosis", that is, they spoke about the simple and colourful maps, graphs, and tables, which makes the project understandable for anyone. Generally, Polish respondents recognized this way of presenting research results as an important feature contributing to the usefulness of research in policymaking.

We found that sometimes Polish policymakers translated scientific information to the policy language themselves to use them in policy process, for example as an input to policy documents. One policymaker said that translation consists of filtering a scientific report to extract the core results. Another policymaker said that cooperation with scientists is needed 
in the translation process; therefore, he meets or talks with scientists in order to extract the usable information for policy. The translated information was usually used to support policy argumentation or action. The translations had a simpler language and included visualizations like tables or graphs with data. Translation was done according to the policy need, so it was a selective use of scientific knowledge. This finding proves that the challenge of conformity with policymakers' knowledge can be easier addressed within a collaborative process (Mode 2).

\section{Quality}

We found that the methodology and credibility of research as well as having 'trustworthy' scientists and their organizations were critical to usability. Polish policymakers paid attention to several aspects of methodology including: reliable data collection; methods for calculations of indicators and finances; monitoring data and its interpretations; use of indicators/factors that describe a certain problem or state of play; issues of spatial and time scales of the research; availability of data sets; and ways of presenting scientific information. All these aspects were valued because they believed that methodology should not be undermined, as that would also undermine quality of results. This suggests that Mode 1 was much is use. Scientific information/knowledge often informed about the state of play, for example regarding pollution of environment, and this in- formation was crucial for deciding on policy actions. Scientific information was thus considered as objective truth that can serve as justification for policy decisions.

Maintaining relations and interactions with scientists have also been mentioned by Polish policymakers as important for the quality of research. Via interactions: (1) doubts towards methodology can be clarified, (2) access to knowledge is easier, (3) there is more understanding about the limitations of science (how to achieve good quality science and present valid results); and (4) justification for certain policy decisions/positions can be stronger if supported by many scientists, representing different research organizations. In practice, contacts with scientists are being strengthened by involving them as experts into policy working groups (examples were present in the Nitrates Directive implementation and programming of rural development policy). To make knowledge more usable, one policymaker mentioned that involving policy officers into research projects as experts, advisors, or members of a steering committee would definitely improve the usability of the final project 
results. This because during the research phase modifications could follow regarding methodology, scope of the research, and data collection, so at the end final results could better fit the policymakers needs.

An example of the use of a good quality scientific report according to Mode 1 can be found at the beginning of the Nitrates Directive implementation. A credible research institute-the Institute of Meteorology and Water Management-by request of the Ministry of Environment (in 2004), delivered a report on the state of play of nitrates concentrations in waters for the whole territory of Poland. This report stated-based on monitoring results from the period 1990 to 99-that there was no serious problem of nitrates pollution, that municipal sewage was the main source of high nitrates levels in surface water, and that the current levels of agricultural activity did not justify the designation of nitrates vulnerable zones (NVZs) (Gorton, Lowe, and Zellei 2005). This information was credible enough to be a basis for a policy decision that nitrates contamination was only a localized problem (which later on proved not to be the case) that could be solved at the local level by local authorities in the regions and farmers themselves. This caused a designation only of a few nitrates vulnerable zones in locations where the problem with nitrates was reported Kowalczewska et al. 2017). In this example, science offered seemingly objective, but ultimately wrong information to decision makers, based on which local actions were planned.

If policymakers have doubts about the quality of research they are reluctant to use its recommendations, especially if this leads to policy change, and they may search for other, more credible expertise on the same subject. During the Nitrates Directive implementation in 2007, an analysis of a foreign, Dutch university (WUR 2007) was published that questioned the Polish designation approach of NVZs. This expertise was not fully based on the Polish national monitoring data; it used other, more local analyses, so it was not clear how the process of data collection was organized, according to one of the policymakers. Due to the questionable methodology of this analysis, Polish policymakers found it difficult to take recommendations for changes of the NVZs designation approach seriously into consideration. So, to validate the proposed recommendations in this analysis (WUR 2007), an additional analysis was commissioned, in this case by policy officers from the Ministry of Agriculture. 
We learned that some aspects of Mode 2 model were also used. A multi-source research was developed jointly (by policy and science communities) within the process of Nitrates Directive implementation after 2007 and used for justification of policy decisions before the European Commission. Almost since the beginning of the implementation process of the Nitrates Directive, the European Commission had been unconvinced with the actions of Poland and intervened several times. These interventions had an impact and led to more multi-level cooperation between institutions, better policy integration, and better integration of research to the policy process. After some time, what was considered quality research offered both instrumental information about the state of nitrates pollution/use and a conceptual frame for revisions of designated NVZs.

\section{Action orientation}

During our interviews, Polish policymakers did not mention action-orientation as a criterion important for usability. Across our investigation, however, we found two strong examples of action-oriented research which produced results for specific situations. In these examples, we noticed collaboration between researchers and policymakers that was helpful in obtaining the expected outcomes. We found there to be no prescribed methodology for action-oriented research, whereas every research focused on different issues/problems and used a different methodological approach. Each example shows that for action-oriented research in environmental policy in Poland, Mode 2 more or less dominated.

One example for action-oriented research related to the design of the rural development measure called 'Adjusting farms to the EU standards' within the rural development programme of 2004-2006. This measure required sophisticated calculations of payments for famers. The idea was to grant farmers a lump-sum payment for modernization and investments at the farm instead of reimbursement of costs. It required the preparation of averaged payment rates for different type of farms: (1) in the case of modernizing these were rates for the purchase of slurry tanks and the construction of slabs, (2) in the case of dairy farms these were rates for the purchase of milking machines and milk coolers, and (3) in the case of poultry farms these were rates for purchase of modern breeding systems. Calculations of payments were ordered and later on delivered by two research institutes belonging to the Ministry of Agriculture. Consultations between policymakers and scientists took place while 
preparing the calculations. Policymakers wanted to have reliable and independent scientific analyses with variations for calculations of payments. The reason of asking two individual scientific institutes for the same analyses of calculations was to consult and validate the calculations in the case of being confronted with allegations or claims regarding unfair rates of payments.

Another example of action-oriented research related to the revision of nitrates vulnerable zones (NVZs) in 2011. The Ministry of Agriculture ordered a special analysis at the Institute of Soil Science and Plant Cultivation in Puławy to support the designation of NVZs. This analysis, based on a model of nitrates outflows from agriculture, designated three different scenarios of unified NVZs designation. Each scenario was proposing a different percentage of areas covered by NVZs and investigated the nitrate contamination problem within specific contexts of agricultural production in different regions. The idea was that policymakers would choose one of the scenarios as a new approach for NVZs designation. The results of this research were presented and discussed at a meeting with policymakers who represented different policy institutions. At the end, none of the proposed scenarios were used. First, there was a concern by some policymakers inside the Ministry of Agriculture that this scientific report delivered by only a single research organization cannot be a reliable basis for the new designation. Second, policy officers (also from different institutions) had doubts about the methodology of this research and the quality of the monitoring data used for the analyses. Third, each of the scenario presented much larger areas covered with NVZs (at the national level) than the current situation at the time, which was not considered desirable from a political point of view (there was no political will to enlarge NVZs in Poland).

\section{Challenging}

In the views of Polish policymakers, the fact whether scientific knowledge was challenging or not was to a certain extent related to funding instruments. Some respondents had doubts if research can be challenging within the Polish science-policy organization of environmental and agricultural domains. Interviewees reported that policy-relevant knowledge production took place mainly in research institutes that are financed by and subordinate to the Ministries (Ministry of Agriculture or Environment respectively) within frameworks of multiannual research programmes or via ad-hoc contracts. Production of knowledge seldom took place at 
Universities or other research organizations (who are financially independent of the two mentioned Ministries) and if it did it was via individual contracts. Policymakers commissioned or sought out scientific analyses by formulating research questions/scopes and science was perceived as delivering a service to policymakers.

Some respondents underlined the fact that some research institutes are so much financially dependent on the Ministries makes it hard to present very critical opinions about certain policies, so knowledge is not challenging. This structure between the Ministry and institutes does promote demand-driven production of science. In the eyes of policymakers, scientists in the past exhibited a more passive rather than active attitude, that is, not fully informing policymakers about national or international research projects in which they were involved in. Thus, some policymakers stated that this financial dependency of research institutes to the Ministries actually pushed scientists to become more active in ensuring the relevance and quality of their work in order to maintain funding. Being active includes organizing conferences for policymakers where scientists present results of their particular research areas (informing about all kinds of projects). Being active includes also being creative and presenting science as inspiration to policymakers.

Polish policymakers were mostly critical of research that tends to criticize the current policy approach, so typical knowledge-driven research produced in Mode 1 was not preferred by them if it did not align with their policy and political interests. According to one of interviewees, such research can be very inconvenient. The implementation of the Nitrates Directive in Poland is a case in point: the first designation of NVZs in 2004 was done by various regions and not in a unified way. There were Polish, peer-reviewed articles criticizing this designation approach of 2004. Polish policymakers did not find this supportive when Polish authorities had to explain and justify the NVZs designation to the European Commission, especially given that the European Commission had many concerns with the Polish implementation of this directive to begin with.

\subsection{Discussion}

Our results show that criteria for knowledge such as relevance, conformity, quality, action orientation, and challenging status quo are still highly relevant when analysing the demands that Polish policymakers make of knowledge and expertise. In the case of Poland and 
environmental policy, we observed that some criteria were more strongly considered than others and that trade-offs occur across different criteria as well. In particular the criteria of relevance and quality were most frequently associated with usability. At the same time, these two criteria were seen to involve a balancing act, as research quality was often assumed to depend on maintaining a distance from policy as in Mode 1 knowledge production, (see also Sarkki et al. 2013; Huitema and Turnhout 2009). The Mode 1 type of research did decrease the chances of producing knowledge that is relevant, as Mode 2 type of interactions and communication between science and policy were for example needed to address the issue of nitrate pollution.

Both Mode 1 and Mode 2 type of science-policy relations were found in environmental policy in Poland. Our analysis shows how preferences for these models were responsive to policy demands related to usability of knowledge. Obtaining relevant knowledge in Mode 1 was rather problematic, so for this knowledge characteristic Mode 2 was preferred. Polish policymakers stressed a lack of knowledge translation, as a part of conformity, due to the use of Mode 1. In terms of action-orientation, policymakers asked for both basic and applied research; for example to give data on the state of play of a pollution problem or to propose solutions to deal with the designation of NVZs. Action-oriented knowledge was thus delivered to certain policy problems and in that sense was more in accordance with Mode 2 types of science-policy relations. Finally, the criterion of 'challenging the status quo' is difficult to categorize under Mode 1 or Mode 2. Hierarchical structures of research institutes under policy institutions imposed a linear science-policy relation, but critique from independent scientists (via peer-reviewed publications) was not appreciated much.

Demands of policymakers related to quality were especially important in shaping their preference for either Mode 1 or Mode 2 science-policy relations. Policymakers' demands for scientific rigour that is considered free of political interferences led to choices for Mode 1 science-policy relations (similar observation: Funtowicz 2006). In Mode 1, science and policy are seen as separate domains, with science perceived as a uniquely neutral provider of objective knowledge (Van den Hove 2007; Wardekker et al. 2008), and decision-making perceived as the domain and responsibility of policy specialists (Demeritt 2006 in Young et al. 2014). Thus, when policymakers consider scientific facts to be true, objective, and independent, they can basically rely on them for the development of policies without the fear 
of political backlash (Valente et al. 2014). Considering science and policy as separated is convenient to policymakers as it gives them room to shift blame and avoid political responsibility (Gieryn 1983; Flinders and Buller 2006). Other times, demands for socially robust knowledge favoured relations and interactions between science and policy as in Mode 2 and other interactive science-policy models. In the process of Nitrates Directive implementation in Poland (after 2007) a multi-source research was developed by policy and science jointly and its results were used as justification of Polish policy decisions in front of the European Commission. So, policymakers' demands were framed in such a way that they steered preferences towards Mode 2, in order to have science support policy decisions in front of a third party.

In our case study, the usability criteria of relevance, conformity, and action orientation proved to be more difficult to obtain in Mode 1. Policymakers acknowledged that Mode 1 has its limitations and will not provide the silver bullet to resolve complex and contentious issues (Mills and Clark 2001). In this context, Mode 2 became attractive to policymakers as it can bring relevant and action-oriented knowledge. Quality knowledge, according to Valente et al. (2014, p. 234), can also be obtained when the client - or policymaker - spells out what is wanted. Increased science-policy interactions may also allow for more open criticism as it could become more constructive and link scientific recommendations with policy actions, although we could not identify this happening in our results.

Our results confirm that neither Mode 1 or Mode 2 types of science-policy relations are panaceas that can satisfy all the demands of policymakers for usable knowledge. While it is the case that knowledge is something better understood when socially co-produced (e.g., Cash et al. 2006), we found important trade-offs in producing knowledge that seeks to be simultaneously credible, legitimate, and relevant (Cash et al. 2003). Sometimes, rushing results to meet pressing policy demands and thereby addressing their relevance involves a risk of less quality, and in turn credibility of the knowledge produced (Sarkki et al. 2013). Equally, increased collaborations with policymakers during the knowledge production process can decrease the problems of value-laden science, by opening up uncertainties and promoting inclusiveness in knowledge production Pielke (2007), but also carries the risk of politicizing science and expertise to the point that it can no longer address policy issues effectively. The reverse can also hold, when knowledge becomes so technicized that its underlying values are 
overlooked, which erodes the social robustness of policy (as was the case when NVZs in Poland where initially determined).

Summarizing, the question of where policymakers' demands for usability could be best addressed in Mode 1 or Mode 2 strongly depended on how these demands were framed and what policy contexts they were parts of. As showed in the example of Polish policymakers, knowledge can be used instrumentally to solve a particular problem such as providing an assessment of the 'state of the environment'. In a closed, politically sensitive environment, it is often considered crucial that knowledge presents an objective truth so that political responsibility is (at least partly) avoided, corresponding to a Mode 1 type of science-policy relations. Such relations however may not hold when pressure to come up with policy solutions mounts, as we saw in examples where the EU intervened on the process of the designation of the NVZs. In response, credible and multi-source research was developed by various policy and research organizations jointly in Mode 2. In sum, we noticed that Mode 1 was predominantly preferred when policy decisions required high quality research (in sense of scientific rigorous) to avoid responsibility in political sensitive issues. Mode 2 was more used when science was used to support certain policy measures because the scientific results were easier to translate, relevant, and action-oriented.

\subsection{Conclusions}

This chapter has illustrated the demands for usable knowledge of Polish policymakers and how those demands shaped preferences for science-policy models. Those preferences, in turn, shape science- policy relations in practice. Thus, the criteria of scientific knowledge that policymakers consider usable are of high relevance for our understanding of why Mode 1 models of science-policy relations often persist as well as why Mode 2 models in other cases are finding their way to reality. Our case study confirmed that interactive and complex sciencepolicy models do serve better to produce knowledge that is considered relevant, actionoriented and conforming with policymakers' background knowledge and beliefs. At the same time, we noticed that other criteria of knowledge-especially quality-strongly link to diverging preferences for either Mode 1 or Mode 2, depending on the context of the policy case. 
While Mode 2 may seem to be a better candidate to respond to multiple demands that policymakers make on the usability of knowledge, it often fails to make a convincing response to policymakers' need to base their decisions on knowledge that is considered objective. Therefore, Mode 1 remains attractive to policymakers, even when it has received considerable-some say even destructive-critiques (Balconi, Brusoni, and Orsenigo 2010). The linearity that is at the basis of Mode 1 supports maintaining the separation of science from policy so that science can be isolated from too high levels of politicization (Metze and Turnhout 2014; Valente et al. 2014) and thus provides what on the face of it appears as objective knowledge on which decisions can be built (Van den Hove 2007; Metze and Turnhout 2014). Policymakers consider this objective knowledge as difficult to be undermined by other parties and therefore a good basis for legitimation of their policy decisions. As long as societal discourse on the role of science in society supports the linear model, the perception of policymakers is that quality and credibility of knowledge remain strongly tied to Mode 1 science-policy relations, even when Mode 2 may offer a type of quality that is more socially robust.

In the end, the choice for one or another science-policy mode depends on the context of the policy case and the particular usability criteria that are deemed important in those contexts. In that weighing of criteria, trade-offs appear to be inevitable. Policymakers are often seen to place most value on relevance and quality of knowledge. While limiting knowledge production to Mode 1 alone decreases production of knowledge that is relevant, balancing trade- offs between relevance and quality can also imply trade-offs between different modes of sciencepolicy relations. Thus, a trade-off between Mode 1 preferences for 'objective' quality and Mode 2 preferences for relevance and robustness may be observed.

Today, most scholars take a normative position that advocates for a shift from the 'traditional' Mode 1 to a 'modern' Mode 2 in order to align knowledge supply with demand and to improve democratic processes. While this position is understandable from an ethical point of view, a more realistic perspective is that Mode 2 will not replace Mode 1 but more likely work in tandem with it (Gibbons et al. 1994; Kazancigil 1998). Policymakers are willing to use both science-policy models to tailor knowledge production processes to their demands for knowledge, making Mode 1 here to stay for at least a little while longer. 


\subsection{List of interviews}

- 7 interviews with seven different policy officers involved in the implementation of rural development policy, Ministry of Agriculture and Rural Development, Warsaw, Poland, April 2007.

- 1 interview with a policy officer involved in the Nitrates Directive implementation, Ministry of Agriculture and Rural Development, Warsaw, Poland, December 2008.

- 1 interview with two regional policy officers responsible for Nitrates Directive in their region, Regional Water Management Authority, Poznań, Poland, December 2008 .

- 1 interview with two regional policy officers responsible for monitoring of waters at the national level, preparing monitoring network for the Nitrates Directive, commenting on national reports of Nitrates Directive implementation, Chief Inspectorate for Environmental Protection, Warsaw, Poland, January 2009.

- 1 interview with two regional policy officers responsible for Nitrates Directive in their region, Regional Water Management Authority, Warsaw, Poland, April 2009.

- 2 interviews with a policy officer involved in the Nitrates Directive implementation and rural development policy, Ministry of Agriculture and Rural Development, Warsaw Poland, April 2010 and December 2016.

- 1 interview with a policy officer responsible for national coordination of the Nitrates Directive, National Water Management Authority, Warsaw, Poland, May 2010.

- 1 interview with a policy officer involved in the Nitrates Directive implementation, Ministry of Agriculture and Rural Development, Warsaw, Poland, September 2016. 
CHAPTER 6

\section{CONCLUSIONS AND REFLECTIONS}




\subsection{Introduction}

The role of science in society has been changing and moving in multiple directions. An important societal trend today is the move towards more complex and interactive models of knowledge production that include users and many other relevant stakeholders. In this thesis, I explored how the European Union (EU) plays an important role in making this trend happen by steering science, policy and society relations. It does so by emphasizing specific needs for co-production of science and policy; for involvement of users and other stakeholders in policy process; and by requiring policies to be based on science of good quality. The EU makes these emphases through various channels: they are made visible in the research culture of the EU and in EU research programming; they are part of EU policy culture and the institutional context of policymaking and policy implementation; and the EU directly promotes specific ideas about how science and expertise should contribute to policy development and effectiveness.

The influence of the EU affects science-policy relations in all member states. At the same time, EU and national research and policy cultures vary significantly across policy sectors and amongst countries. Poland is a country that in particular has struggled to comply with EU environmental policy requirements and has already faced several legal procedures as a result. The case of Poland is exemplary of how many Central and Eastern European (CEE) countries deal with EU environmental policy, where many national policy processes as well as sciencepolicy relations today are being steered to an important extent by EU policy processes.

This thesis investigated how the implementation of the EU environmental and rural development policy has shaped science-policy-society relations and the use of knowledge in Poland. This included an exploration of how policy demands articulated by the EU and by national users affect science-policy relations and of the multiple directions in which these science-policy relations change. The thesis addressed the following main research question:

- How are science-policy relations in environmental and rural development policy in Poland shaped by EU policy processes?

And the following three sub-questions: 
A: How do European and national policy culture and institutions shape science-policy relations?

B: How are science-policy interactions managed and/or steered in specific policy projects and programmes?

C: How do user and policy demands affect science-policy relations?

The next section of this chapter provides answers to the sub-questions and the main research question. Subsequently, it offers a theoretical and methodological discussion based on the findings. These include: i) a debate on the changing role of science and the shift from Mode 1 to Mode 2; ii) a debate on the imperfect shift towards Mode 2 in Poland; iii) a debate on the role of knowledge and expertise in Europeanisation processes; and iv) a reflection on the case studies and my own role as a researcher who is also active as a practitioner in these cases. The chapter concludes with a call for reflection on science-policy relations and some recommendations for both policymakers and experts on how to manage science-policy relations.

\subsection{Answering the sub-questions}

\subsubsection{Sub-question A: How do European and national policy culture and institutions shape science-policy relations?}

Sub-question A was specifically explored in Chapter 2, which presented the historical analysis of the Nitrates Directive implementation in Poland. Four main conclusions are to be highlighted:

1. The European Commission (EC) expects member states to converge as much as possible with EU policy and administrative arrangements. The results showed that policy convergence in Poland is a process that proceeded over time, resulting in the adoption of EU law (like the EU Nitrates Directive), administrative structures, and styles. Although a general trend was observed towards policy convergence, this process of convergence does not always proceed very smoothly. The results showed that policy convergence is being hindered by domestic issues related to institutional and cultural contexts (e.g., hierarchical administrative structure, poor communication 
between different institutions within policy and science, political dynamics), and that these hindrances still exist today.

2. The top-down nature of both the Europeanisation process and EU environmental policymaking strengthened existing knowledge and expertise and added new demands for knowledge and expertise within the science-policy interface. The EU environmental directives, such as the Nitrates Directive, target the science-policy interface directly by calling for specific expertise related to the establishment of environmental zones, the identification of pressures, and measurable and verifiable actions. In addition, the EU's monitoring and control mechanisms and the EC's interventions within the implementation process oblige member states to enforce effective environmental actions and report on progress towards improving the environment. These top-down dynamics gave an impulse to a clear research demand to which Polish policymakers responded by approaching their own - already trusted and considered credible research institutes directly. In sum, it strengthened the pre-existing top-down science-policy relations in Poland.

3. Institutional and cultural changes in science-policy relations developed over the years due to active EC's interventions and pressures on Poland towards compliance with the EU Nitrates Directive. The implementation process started from a situation of clear misfit, where Polish institutions and the Polish culture of expertise were misaligned with the Directive's requirements. First, this was because the ministries of environment in CEE countries, including Poland, were often politically and administratively powerless as a heritage of previous political systems (communism, socialism) (Kramer 2004). Second, Poland had a less pluralized policy apparatus in comparison with the EU, weak institutional cooperation, and a different tradition of knowledge production and use (including a mainly linear science-policy interface) (Kowalczewska, Behagel, and Turnhout 2017 ; Zybała 2013 ). Subsequent years of Nitrates Directive implementation showed slow but increasing policy convergence. This included the development of the necessary institutional cooperation for environmental monitoring and assessment, strengthening the position of environmental administrations, and increasing demand and use of science. Thus, science-policy relations became more interactive. Importantly, personal networks of 
policymakers and scientists, as well as more structured interactions, developed over time as well.

4. Cultural contexts tend to be a more persistent obstacle in meeting the EU requirements than institutional contexts (Kowalczewska, Behagel, and Turnhout 2017). Cultural contexts may either prolong the process of alignment of science-policy relations with the EU civic epistemology or stall it. Accordingly, the future convergence of EU and Polish cultures of expertise towards more interactive science-policy interfaces within the Nitrates Directive implementation in Poland is difficult to predict. The risk of top-down approaches towards policy change is that once a pressure is gone or domestic political dynamics change, both institutional and cultural changes may reverse (Shiers et al. 2014). Recent political changes at the national level in Poland appear to point to such a reverse direction of policy change towards previously used top-down and linear models of science-policy relations.

\subsubsection{Sub-question B: How are science-policy interactions managed and/or steered in specific policy projects and programmes?}

Chapters 3 and 4 investigated how science-policy interactions are managed/steered in policy projects and programmes. The findings have led to the following three conclusions:

1. Knowledge brokers can play an important role in managing science-policy relations by aligning the supply of knowledge with the demand. Chapter 4 discussed the specific example of how a knowledge broker managed interactions between science and policy in one of the EU public procurement contracts. The knowledge broker employed specific knowledge brokering activities related to the planning, control, and monitoring of the knowledge production process (see also in Munns and Bjeirmi 1996). These activities focused on communicating research needs to knowledge suppliers so that they would meet these needs and provide knowledge outcomes that are useful to policymakers. A variety of communication tools, including external workshops with potential users/policymakers from member states, was also found helpful to align research results with policymakers' demands. In addition, knowledge brokering activities focused on building trust between all involved in the knowledge production process through repeated interactions and on deepening an understanding of each 
other's perspectives and research limitations. Both trust and understanding were found to be crucial for successful science-policy relations (Saarela et al. 2015; Gaudreau and Saner 2014).

2. Boundary objects play an important role in managing science-policy interactions by bringing actors with different backgrounds together. Chapter 3 discussed how a computer-based scenario model called EUruralis was able to perform as a boundary object. The Eururalis project encouraged policymakers and scientists from different member states and different disciplines to discuss the future of agriculture and rural areas in Europe by presenting scientific data and methodologies in four different scenarios. By doing so, it offered common terminology and vocabulary which stimulated not only human interactions but also interactions of users with the EUruralis computer tool. In addition, interactive boundary objects like EUruralis may stimulate further learning. EUruralis proved to be a good learning experience for scientists of different disciplines who cooperated and connected each other's models into one computer-based programme. It allowed policymakers to experience how to develop multidisciplinary and participatory projects. The most important learning element for both policymakers and scientists was found in the way EUruralis was able to facilitate policy discussions, reflections, and co-production (Kowalczewska and Turnhout 2012).

3. Early engagement of users in projects/programmes is important. Involving policymakers in the early design phase of the EUruralis project resulted in a tool that was much appreciated by policymakers because of its policy relevance and its attractive way of presenting the scenarios (simple and colourful maps, graphs, tables, and contrasting scenarios). The task of policymakers was to assist scientists in the modelling process by verifying the data and to help formulate research questions which EUruralis was supposed to answer. In the EU public procurement contract (Chapter 4), policymakers also started to interact with researchers very early on to ensure the alignment of research to their needs, that these needs had been well understood, and that requested knowledge production was feasible. 


\subsubsection{Sub-question C: How do user and policy demands affect science-policy relations?}

Sub-question C was discussed in Chapter 5, which analyses how different policy demands, categorized by means of the usability criteria of Weiss (1995), affect the way in which Mode 1 and Mode 2 science-policy models are employed. Chapter 5 uses the notions of Mode 1 and Mode 2 as an umbrella concept to discuss policymakers' preferences for either monodisciplinary and linear or more complex and interactive science-policy models. The analysis focused on two policy fields in Poland - rural development and nitrates policies where both Mode 1 and Mode 2 models were found to be relevant (Kowalczewska and Behagel 2018). Four conclusions are highlighted:

1. The preferences of Polish policymakers for either Mode 1 or Mode 2 science-policy models corresponded with different sets of usability criteria. Mode 1 better satisfied the criteria of quality, in the sense of scientific standards related to methodology (Weiss 1995), and conformity. Demands for a scientific standard where knowledge is considered free of political interferences led to choices for Mode 1 because it allowed policymakers to treat science and policy as separate domains (see Funtowicz 2006 for a similar observation), to consider science as a uniquely neutral provider of objective knowledge (Van den Hove 2007; Wardekker et al. 2008), and to leave responsibility for decision-making in the hands of policy experts (Hoppe 2010; Demeritt 2006 in Young et al. 2014). Mode 1 also corresponded with the criterion of conformity, because knowledge translation processes, which require an ongoing collaborative process (Shonkoff and Bales 2011), were not well developed in Poland. Mode 2 better satisfied the criteria of relevance and action orientation. Interactions between policymakers and scientists were considered crucial for achieving relevant research, which in this case meant that it was in line with EU law and well-timed to fit policy implementation timelines. Action-oriented research was carried out for specific policy problems, for example, to design a rural development measure or to develop a unified methodology for the designation of nitrate vulnerable zones for the purpose of the Nitrates Directive. In these examples, collaboration between researchers and policymakers, as advocated in Mode 2, was helpful in achieving the expected outcomes. 
2. Second, different interpretations of quality led to different preferences for either Mode 1 or Mode 2. One interpretation of quality included references to scientific standards related to methodology. Polish policymakers paid attention to several aspects of methodology, including reliable data collection; methods for calculating indicators and finances; monitoring data and its interpretations; use of indicators/factors that describe a certain problem or state of play; issues of spatial and time scales relevant to the research; and availability of data sets. Methodological aspects were valued because policymakers believed that methodology should not be undermined, as that would also undermine quality of results. All of this points to Mode 1. A different interpretation of quality - where quality is understood as following peer review - required more interactive science-policy relations (like Mode 2). This involved both the inclusion of scientists from different disciplines and the inclusion of different knowledge types (including non-scientific) to produce more socially-robust knowledge (Nowotny, Scott, and Gibbons 2003). Within the process of the implementation of the Nitrates Directive, socially-robust research was developed to offer both instrumental information about the state of nitrates pollution in waters due to agricultural production and a conceptual frame for a revision of the designated nitrate vulnerable zones. This Mode 2 type research was used to justify policy decisions with respect to the EC.

3. There are trade-offs between the studied usability criteria. One of the reasons for these trade-offs is that neither Mode 1 nor Mode 2 types of science-policy relations are panaceas that can satisfy all of the demands of policymakers for usable knowledge (Kowalczewska and Behagel 2018). Sometimes, rushing results to meet pressing policy demands and thereby addressing their relevance involves a risk of reduced quality, which in turn weakens the credibility of the knowledge produced (Sarkki et al. 2013). Equally, increased collaboration with policymakers during the knowledge production process can help open up debates on uncertainties and promote inclusiveness in knowledge production (Pielke 2007), but also carries the risk of politicizing science and expertise to the point that it can no longer address policy issues effectively. The reverse can also hold true. When knowledge becomes so technicized that its underlying values are overlooked, this can erode the social robustness of policy. 
4. The difference between political or instrumental intentions for the use of knowledge was an important factor in shaping the preferences of Polish policymakers for different science-policy models as well. When Polish policymakers needed instrumental information about the state of environment, they could use either Mode 1 or Mode 2, depending on the intentions. Within an instrumental perspective on knowledge, science delivers empirical evidence and conclusions that can, in principle, help to solve a policy problem (Weiss 1979). Close interactions, a characteristic of Mode 2, were considered crucial for achieving relevant research. Once demands of knowledge are linked with politically sensitive contexts of use, knowledge provides ammunition in political arguments (Wesselink et al. 2013). In this situation, it becomes congenial when knowledge is difficult to undermine. In such a politicized context, policymakers can benefit from scientific knowledge that is isolated from interactions with policy, resulting in a preference for Mode 1. Such science-policy relations may, however, not always hold when pressure to come up with policy solutions mounts. This was exemplified in Poland within the Nitrates Directive implementation where EU interventions on the process of designating the nitrate vulnerable zones impacted science-policy relations and changed them from a predominant Mode 1 model, which did not fit well with the EU, to a model resembling Mode 2.

\subsubsection{Main research question: How are science-policy relations in environmental and rural policy in Poland shaped by EU policy processes?}

This thesis showed that science-policy relations in environmental and rural development policy in Poland are shaped by EU and national institutional and cultural contexts (sub-question A), the process of managing interactions (sub-question B), and specific aspects of policy demands (sub-question C). In addition, important links were found between: i) EU policy processes and policy demands at national level, ii) those national policy demands and the choices for science-policy models and hence different management styles, and iii) EU and national institutional and cultural contexts and preferred type of science-policy relations. Each point is explained below. 
First, EU environmental directives and regulations were seen to strengthen the existing top-down structure for policy-driven knowledge in Poland while at the same time creating new knowledge demands. Polish policymakers needed to respond to legal requirements of the EU as a part of the policy convergence process. Such requirements were, for example, set out in the EU Nitrates Directive and rural development regulations. These top-down requirements strengthened the existing top-down institutional structure for ordering research from research institutes that are internal to the ministry. New demands to be fulfilled by national authorities were also introduced. Specifically, assignments like the designation of environmental zones required monitoring and reporting efforts that were structured by common and standardized categories (Waterton and Wynne 1996). These EU requirements steered Polish policymakers' knowledge needs and resulted in some institutional and cultural changes. Polish policymakers wanted high quality and relevant knowledge that could be readily used to decide on actions. The urgency of these demands helped push towards more interactive science-policy relations and more open communication, thereby changing the culture of expertise. Polish policymakers also wanted knowledge that could not be undermined by a third party, such as the European Commission, and that would hold when they would be asked to defend policy implementation choices. To achieve this, it was important that science and policy were closely intertwined and presented a shared message.

Second, changing policy demands required specific forms of management of science-policy relations to addresses those demands. This was illustrated in Chapter 5, which described how different policy demands for usable knowledge steered choices of policymakers towards either Mode 1 or Mode 2, and thus required different management of science-policy relations. The demands of Polish policymakers, formed by both national and EU contexts, related to different criteria of usable knowledge, such as relevance, conformity, action orientation, and quality. When specific criteria were given extra importance (i.e., relevance), this required active management of the science-policy interface towards Mode 2 (for the case of relevance). Moreover, criteria could be interpreted differently: quality was either seen as methodological rigor or as extended peer review, and those different interpretations also required different types of science-policy relations. Specifically, demanding scientific facts to be objective and independent called for a Mode 1 type of relation. Alternatively, demands for socially robust knowledge (that is also relevant and 
action-oriented) favoured relations and interactions between science and policy that fit with Mode 2.

Third, the EU has its own cultural and institutional ways of managing science-policy interactions in the environmental policy field and these have translated into a preference for a specific science-policy model that is at once interactive, participatory, and multi-level (Newig and Fritsch 2009). By implicitly following this EU model, member states are expected to achieve effective policy implementation. In empirical practice, the effectiveness of this EU model remains disputed (Newig and Fritsch 2009, p. 198). Newig and Fritsch (2009) show that hypotheses related to the effectiveness of different environmental policymaking models (e.g., participatory versus top-down models, local versus higher scale decision making, and multilevel governance) require further research and need to be placed in the context of a specific country. Whereas there appears to be an EU-wide consensus that science is a part of policy processes, the degree of scientific involvement and roles assigned to science will differ per country. Poland does not follow this ideal EU model completely, nor does it have a fully developed participatory policymaking model, and so it struggles with the implementation of EU environmental law. As long as the EU continues to push towards its own ideal EU model within the EU environmental policy, including a science-policy model, clashes between EU and Poland will continue.

\subsection{Revisiting the changing role of science and the shift from Mode 1 to Mode 2}

\subsubsection{The shift from Mode 1 to Mode 2 is incomplete}

The changing role of science in society is strongly related to the changing demands and perceptions of society and policy towards science. Over the last decades, society and decision makers/policymakers increasingly came to expect that science would provide directions and solutions for complex challenges where there is uncertainty about the nature of the problem, their solutions, and outcomes. Accordingly, it is becoming increasingly visible that both society and policy cannot be divorced from science (Van Wyk et al. 2007). "Science should be seen as an inextricable part of a political process, rather than as an external input for policy processes" (Turnhout 2003, p.135). This statement is at the core of the discussion about the changing role of science. That discussion has been developing for quite some time now, including 
contributions from Weinberg (1972) who wrote about a necessary shift from science to transscience, Funtowicz and Ravetz (1994) who wrote about a shift from science to post-normal science, and Gibbons at al. (1994) who wrote about the shift from Mode 1 to Mode 2. Especially the latter shift elaborated by Gibbons et al. (1994) has been discussed in this thesis.

Gibbons et al. (1994) and Nowotny, Scott, and Gibbons (2003) proposed the concepts of Mode 1 and Mode 2 and provided an influential description of the changing role of science and changing science-policy and society relations. The concepts of Mode 1 and Mode 2 have been used in this thesis as an umbrella concept to discuss different science-policy models, either monodisciplinary and linear or more complex and interactive. As discussed in the introductory chapter, this thesis has considered the Mode $1 /$ Mode2 discussion to be embedded in multidirectional changes in the theory and practice of knowledge production and use. This thesis highlighted that the most visible change throughout all its presented case studies is the direction towards co-production, which is a management model for science-policy relations that today has been embraced by many researchers, stakeholders, and funding organizations to solve sustainability problems (Lemos et al. 2018).

Despite a general trend towards more complex and interactive models like Mode 2 and co-production, the examples included in this thesis show that the shift from Mode 1 to Mode 2 is not unidirectional. Rather, this shift is imperfect or incomplete and it plays out in the context of other developments that continue to pull science towards Mode 1. Such imperfect shifts are also known in the governance literature, which has provided multiple examples of multidirectionality in the shift "from government to governance" (see also Boonstra 2004, Van der Zouwen 2006). Van der Zouwen (2006) showed that in policy processes in which nongovernmental actors are involved, governmental actors can still dominate and determine who takes parts and with what task/role, making a shift towards multi-actor governance imperfect. Analogously, a shift towards Mode 2/co-production will also remain imperfect and sciencepolicy interactions may continue to fluctuate between Mode 1 and Mode 2/co-production as these models are found in use today for different reasons in Poland and elsewhere. Moreover, within Mode 2 science models, elements of Mode 1, such as the idea of a separation between policy and science, may remain dominant. 


\subsubsection{In between Mode 1 and Mode 2}

By applying concepts from the field of STS, governance studies, and Europeanisation studies, this thesis has offered four explanations of why the shift towards Mode 2 is imperfect and may be difficult to achieve in Poland and possibly also beyond. First, both Mode 1 and Mode 2 science-policy models are attractive to policymakers. Mode 2 is acknowledged by many policymakers as better in delivering relevant, legitimate, and credible knowledge (as defined by Cash et al. 2002; 2003). As such, increased science-policy interactions can create knowledge that fits the needs of policymakers (Valente et al. 2014), with the help of knowledge brokers and boundary objects. This may also allow for more open criticism that can be used as constructive feedback while linking scientific recommendations with policy actions. At the same time, policymakers' demands for scientific standards and for knowledge that is considered free of political interferences often leads to choices for Mode 1 (see also Funtowicz 2006). Moreover, Mode 1 is still supported by many governmental institutions and policymakers today (Kraak 2000). Linear science-policy relations are convenient for policymakers as they give them room to shift blame and avoid political responsibility for decisions (Gieryn 1983; Flinders and Buller 2006). For example, Wesselink et al. (2013) discuss how global climate change governance is built on a linear approach to science-policy interactions. Such a linear approach, once established, is difficult to overcome and it requires conscious and explicit efforts to do so, as is for example the case for the International SciencePolicy Platform on Biodiversity and Ecosystem Services (IPBES) (Turnhout et al.2013).

Second, knowledge production and use are both embedded in cultural and institutional processes. Accordingly, they change in tandem with broader policy changes. For example, Zybała $(20,13015)$ points out that both cultural aspects and structures of public administration in Poland make participation in the process of knowledge production and use within public policies rather problematic. Still, the analysis presented in Chapter 2 showed that changes in science-policy relations in Poland are possible and were reported when implementing the Nitrates Directive. These changes in science-policy relations were indeed accompanied by mutually constitutive institutional and cultural changes, also expressed in the idea of "infrastructures of expertise" (Halfmann 2003; 2005). However, the analysis also showed that in Poland these instructional and cultural processes are rather resistant to 
change, which means that shifting science-policy interactions towards Mode 2 and co-production takes time.

Third, aligning supply and demand of knowledge is a challenging process that involves complex social processes. These processes therefore require suitable facilitation and joint efforts. Many scholars highlight communication problems and other difficulties in science-policy relations (e.g., Johnston and Soulsby 2006, Turnhout, Hisschemöller, and Eijsackers 2007; 2008, Sarewitz and Pielke 2007). Valente et al. (2014) have pointed out that the quality of scientific advice also depends on the clarity and quality of policymakers (so the demand side) in being able to identify and communicate what their needs and requirements are. Therefore, organizations put a lot of emphasis on facilitating knowledge exchange between science and policy with the help of knowledge brokers or boundary objects (Carlile 2002; 2004; Ward, House, and Hamer 2009). Chapter 4 brought attention to the role of the knowledge broker performed by an experienced policy officer who tailored his knowledge brokering activities to specific policy problems and directed them to reconciling supply and demand of knowledge. Likewise, the analysis of Chapter 3 confirmed that boundary objects play a role in bringing people with different backgrounds and knowledge together by providing them with a common vocabulary and offering space for learning and reflection (Kowalczewska and Turnhout 2012). This investment may involve the organization of specific training programmes for policy officers who work with research daily and who can benefit from a better understanding of science, including its limitations. Other examples from the literature show that not only workers in the private sector are being trained to become knowledge brokers (Ishiyama 2016), but also public policymakers who are tasked to improve science-policy dialogues (Bielak et al. 2008).

Fourth, public participation and the opening up of policy processes to include types of knowledge other than scientific still require more attention and acceptance in some countries. It is especially important to reflect on the participation and inclusion of alternative types of knowledge in the context of the shift towards Mode 2. Not only scientists and elite experts, but also a more temporary and heterogeneous set of independent participants are considered important in policy processes because they can complement scientific knowledge and sciencepolicy interfaces with both additional and alternative knowledge and insights (Van Bommel 2008). However, as reported by Paloniemi et al. (2015) public participation in biodiversity 
governance in Poland is still rare and when implemented this is mostly due to legally binding legislation. Public participation and the engagement of different types of knowledge may thus differ among different sectors and policy themes within the country. Turnhout, Hisschemöller, and Eijsackers (2007) - while discussing ecological indicators - shows that "even though indicator development is demand driven, interdisciplinary, uncertain and value laden" (p 225), scientific knowledge is still dominant in the development of ecological indicators and the inclusion of perspectives of other stakeholders requires acceptance, which suggest that this is still uncommon. Jasanoff (2003 p. 162) explains this continued dominance by pointing to the ongoing refusal to think systematically or theoretically about the changing role of experts and expertise in our legal and administrative systems. In addition, democratic structures in some countries may lack the institutions to facilitate participation in knowledge production (Hisschemoller 2004). As a result, the power and dominance of scientific knowledge remains because other types of knowledge from other participants simply are not available or not included.

\subsection{Revisiting the concept of Europeanisation}

A key finding of this thesis is that science-policy interfaces play a central role in policy convergence processes related to Europeanisation. Traditionally, policy convergence is often explained by referring to the extent of institutional "fit" between a member state and EU requirements (Knill, Tosun, and Bauer 2009; Mastenbroek 2005; Frederiksen et al. 2017). According to this "goodness of fit" theory, policy convergence will progress smoothly for a member state whose existing institutions at the national level fit to those of the EU, while others will struggle more and may end up achieving lower levels of convergence. Knill and Tosun (2009, p. 874) wrote that in exploring how the EU matters, many scholars have focused their research on issues of policy convergence and the implementation of the European directives and regulations (for example Knill 2001; 2005; Heritier et al. 2001; Knill and Lehmkuhl 2002; Olsen 2002; Börzel and Risse 2003). I argued in Chapter 2 that scientific expertise is an important and under-researched factor that influences policy convergence processes within the Europeanisation context. Especially for countries like Poland, which have not been in the position to shape EU environmental policy (on topics like biodiversity, water, and so on) but mainly "download" it, the production of knowledge and expertise was a key part of policy convergence. 
For countries like Poland, but also other countries in Central, Eastern, and South-eastern Europe, the process of Europeanisation of policies, institutions, and expertise remains a puzzle. Around the time of the last two EU enlargements in 2004 and 2007, many scholars pointed out that new member states would not be able to follow and adapt to the EU environmental policy requirements (Carmin and Vandeveer 2004) and therefore their EU membership constituted a threat to ambitious EU environmental policy. This assumption turned out to be incorrect as CEE countries do not perform worse than many "older" member states when it comes to the "formal implementation" of the EU environmental law (Braun 2016, Sotirov, Lovric, and Winkel 2015, p. 988). Specifically, CEE countries prepared their institutional frameworks for legal transposition of the EU legislation relatively well. The case of the Nitrates Directive in Poland confirmed that institutional competences were adapted to implement the directive via national legal acts in a straightforward manner. The phases of practical implementation and enforcement turned out to be more problematic. Practical implementation is defined as a process in which "state authorities provide administrative resources to put policy objectives into practices as well as monitor, encourage, or coerce rule-consistent behaviour of public and private actors as regulatory targets by incentives and sanctions" (Sotirov, Lovric, and Winkel 2015, p. 988). Thus, policy convergence involves ongoing social processes that go beyond formal and institutional change.

By involving social processes that include knowledge production and use, the practical implementation of EU environmental policies brings complexity and gives rise to conflicts for which CEE countries seem to be less prepared. Many social processes are culture-specific and so more resistant to change. These processes involve science and policy cooperation, broadening stakeholder participation, policy management, and coordination. Moreover, they may include the politicization of policy problems and struggles between different stakeholders and their interests. Such social processes may "give rise to new disparities and new forms not only of cooperation but also competition, create winners and losers in policy processes that, in turn, constitute political potential for articulation of conflicting interests and demands by political parties, interest groups and social movements" (Cianciara 2017, p. 241). In sum, policy convergence creates social dynamics around knowledge production and use that includes struggles over both the instrumental and political role that knowledge can play. 
There is still a scarcity of systematic studies on Poland and other CEE countries regarding how they practically manage the implementation of EU environmental legislation (Braun 2016) and what social processes accompany these implementations. Such studies would bring valuable insight to help along further debates about what Europeanisation entails in practice and to what extent and how this can be translated to the process of socialization of member states and their societies to the EU. As shown in the Nitrates Directive case, there are social processes ongoing in Poland as a result of the Europeanisation process that depend on the domestic institutional fit or misfit with the EU and have a strong cultural dimension as well. The historical analysis of the Nitrates Directive's implementation revealed cultural changes within the science-policy relation in Poland as an integral part of Europeanisation. Hence, it is worth to consider including questions about the role of science and expertise in Europeanisation studies, how different countries adapt their science-policy interfaces, and what the roles of national culture is in these processes.

\subsection{Methodological reflection}

The case study approach of this thesis has allowed for a better understanding of how implementation of the nitrates policy and rural development policy shaped science-policy relations and the use of knowledge in Poland. These two policies were chosen because from 2004 to 2016 a considerable amount of knowledge and expertise was required and produced within these policy initiatives. This has dynamically influenced science-policy relations. Various research institutes provided descriptive and analytical parts of these policies and their measures by delivering an analysis of a variety of indictors, as well as statistical and monitoring data. In addition, these policies were subjected to strong political dynamics, including between the EU and Poland (Kowalczewska, Behagel, and Turnhout 2017). This was especially visible during the Nitrates Directive implementation process, where the intervention of the EC and its critical evaluation of the Polish implementation approach made the Nitrates Directive become the focus of broader political and administrative attention in Poland.

One could argue that a limitation of this research project is that it focuses only on nitrates and rural development policy. Indeed, the analysis of a different EU environmental directive could have led to different results. Had the research project focused on, for example, the Water Framework Directive or Natura 2000, this thesis would have likely placed more focus on 
stakeholder participation. Especially more recent EU directives, like the Water Framework Directive, are part of what some call the "participatory turn" in EU environmental policy (Saurugger 2010), which includes a more active call for stakeholder participation and transparency in reporting, amongst other things. This would allow for a stronger emphasis on another dimension of the Europeanisation process related to broadening the policy processes for participation of various stakeholders. At the same time, it may have distracted from the central role that science and expertise play in these and other directives, as political conflicts and implementation struggles are often most visible in stakeholder arenas, even if they originate elsewhere.

Data collection methods included interviews, participant observation, and document analysis. I collected the data while working at three different places: i) the Polish Ministry of Agriculture; ii) the European Commission, DG for Climate Action; and iii) Wageningen University and Research, Landscape Department. In the first two places I worked as a policymaker on policy files related to nitrates and rural development. While interning at Wageningen University and Research, I observed the EUruralis project and learned how to develop a joint science-policy project. All of these work experiences were helpful in identifying interviewees, asking them for their time and information, and gaining their trust. However, having an insider perspective also raised a challenge: to make sure my interviewee data was not coloured by my own biases. I sometimes struggled with avoiding becoming too policydriven in preparing, conducting, and interpreting interviews and colouring the interviews with my own policy perceptions and understandings. I also struggled with taking a step back as a policymaker and taking on the role of meeting observer in order to avoid confusing analytical standards with normative opinions. To deal with this, I was very careful to include substantial analysis of policy documents in my research and did not depend on interviews alone. I have read and reviewed many policy documents that span the period 2004 to 2016. In these official and written policy documents (notes, letters, reports, etc.) as well as scientific reports, I was looking for a confirmation or refutation of what I found in interviewee and participant observation data, and vice versa.

While conducting research I discovered that Polish policymakers working on both the nitrates and rural development policy have been reflecting on science-policy relations in Poland themselves. These reflections touched on the roles of research organizations and funding in 
Poland, which promote demand-driven production of science, amongst others. Policymakers were wondering if Polish research institutes that are financially dependent on different ministries may generate very critical opinions about policy proposals of these ministries. On the other hand, according to some policymakers, the dependency of research institutes on the ministries also pushed scientists to become more active in ensuring the relevance and quality of their work and in maintaining funding. Being active included, for example, organizing conferences for policymakers where scientists presented the results of their projects as they relate to nitrates.

The opportunity to work and collect data in three different environments, where I practiced different policymaking models and science-policy relations like Mode 1 and Mode 2/co-production, was a great learning experience. I experienced that science-policy relations based on engagement, understanding, interactive communication, and willingness to solve jointly policy problems are more effective in generating usable outcomes. In addition, I noticed that policymakers in the EC put lot of emphasis on clearly formulating policy problems and needs as well as on having extensive consultation processes both with the policymakers working for different departments/sectors and with other relevant stakeholders, including representatives of member states and non-state actors. My learning experience led to a change of my own cultural policy beliefs to acknowledge that collaborating to coproduce knowledge is conductive to effective environmental management and policies. I also agree with the claim of Fernández $(2016$, p. 174) that open consultation and exchange is an essential process that aims improve everyone's environmental literacy in the broadest sense.

The belief in collaborating to co-produce knowledge impacted my research both conceptually and practically. I was looking for positive examples on what co-production may look like in practice and how it can be successful. Therefore, this thesis included practical-orientated explorations of the systems and procedures that might help on the science-policy frontier, presented in Chapter 4. It explored the value of the knowledge broker who acted as an intermediary and translator to successfully facilitate knowledge co-production in one EU public procurement contract. This conviction also gives meaning to my current work as an agricultural policy advisor at the Dutch Embassy in Warsaw, Poland. Via embassy activities in Poland, I promote different forms of cooperation, including 
public-private partnerships and multi-actor approaches by drawing on examples from the Netherlands. Examples of cooperation include the triple helix (cooperation of knowledge institute, governments, and industry), the Wageningen approach (cooperation of natural and social sciences as well as specialized research institutes and the university), Polish-Dutch science cooperation, and science-industry cooperation more generally. Cooperation between research institutes and different types of actors is still uncommon in Poland, but growing awareness and calls by different stakeholders for strengthening cooperation on different levels are more visible today than several years ago.

\subsection{Recommendations}

As the final point in this thesis, I would like to make a call for scientists, policymakers, and other stakeholders to be more reflexive about the role of science and science-policy-society relations in the context of environmental and rural development policy in Poland. While the EU has been searching for the rightful place of science in policy and society, we need to engage in a similar process in Poland. This is a process in which a number of aspects need to be reflected on, and keeping course of shifting science-policy relations in the direction of co-production is important. There is an increasing understanding within scientific and policy communities as well as different stakeholders worldwide that collaborating to co-produce knowledge will increase its use (Lemos et al. 2018). That does not happen on its own. Co-production of knowledge needs to be familiarized, understood, tested, and reflected on if it is to contribute to effective policymaking and to be supported in Poland. There is already a growing number of scientists and potential users who can deliver examples on how to organize co-production and give insights about costs, time, and participation (Lemos et al. 2018). This knowledge should be acquired (this thesis is an attempted starting point) and discussed.

The concept of usability is becoming dominant in debates on science, policy, and society relations and knowledge (co-)production in the environmental field. It also leads to reflection on the complexity of knowledge systems where science and practice interact. As participants within the knowledge systems represent various institutions and domains, they have different interpretations of the nature and extent of the credibility and salience required when producing knowledge (similar observation in Ingram et al. 2016). Today, environmental policy 
implementation is no longer understood as a linear application of a set of external objectives (Behagel, Arts, and Turnhout 2017), but as a dynamic process of interpretation and negotiation where science should be seen as one of the important actors but other participants and types of knowledge should not be excluded. In that context, policymakers should give more prominence to activities in the policymaking process that focus on managing and taking into account stakeholder feedback, expectations, and views, as well as negotiating how to reconcile different stakeholder needs. This may bring more understanding of policy problems and more effective processes of knowledge co-production and exchange while searching for the usable policy responses.

Reflecting on types of research organization and forms of funding is important as this relates to the effectiveness of knowledge production systems. It is about steering towards more formal or informal, hierarchical, corporate or co-productive science-policy relations. Reflection should be given on funding and capacities of current research organizations in Poland in facilitating co-production. Poland follows a model where universities and scientific institutes focusing on basic and applied research in environmental and agricultural sciences are detached from each other and where disciplines are kept separate. Public funding of research that is dominant in Poland is seen "as a set of exchange relationships between different types of funding agencies - including ministries, research councils, other agencies and a (usually larger) set of research performers who provide research services" (Lepori et al. 2009, p. 669). These relationships are still highly institutionalized in a form of hierarchical structures (Lepori et al. 2009). Public funding of research also includes different types of instruments (e.g., core vs. project funding). It is important to reflect on how to manage different funding streams between different research organizations (universities, research institutes), different expected deliverables, and how to combine financing with enhancement mechanisms that will push scientists to closely collaborate with other stakeholders while engaging in knowledge production processes. The collaboration of scientists with other stakeholders can link scientific knowledge to practice and the context of use, and thus help solve real problems for real people. This direction has been stressed by Sarewitz (2016, p. 39) who argued that "in the future, the most valuable science institutions will be closely linked to the people and places whose urgent problems need to be solved". 
New societal demands put pressure on scientists worldwide to integrate scientific disciplines and to become more proactive by speaking to people, showing their research results in a simple way that lay people can understand, and addressing the value that scientific innovations may have for society (Felt 2017). There is a need for scientists from different disciplines to work together, which can only result in better conceptualizations of research and projects (Kebo and Saner 2014). There is also a need to develop the domain of science communication for non-experts. In addition, scientists need to reflect on what is considered good science and how a good researcher should be (Felt 2017). Their views should be shared and discussed with policymakers who also have views, often different, on what is good science for them and how a good researcher should be. Mutual engagement and understanding between scientists and policymakers are an important basis for co-production.

Finally, just like scientists need to reflect on what knowledge they supply, policymakers need to take more responsibility for identifying and communicating what their needs and requirements towards science are. The quality of scientific advice also depends on the clarity and quality of the way in which demands and research needs are expressed and communicated (Valente et al. 2014). Moreover, it depends on the effort that is put into coordinating and facilitating interactions between knowledge production and use. For example, one could explore introducing special teams in policy organizations responsible for the science-policy interface, who coordinate and translate policy needs into research questions, discuss with scientists the details of their research, are part of research teams to provide feedback, and translate scientific results to a language understandable to policymakers. In conclusion, there is a need for more strategic thinking about the ideal science-policy relation model for each country, the model that best suits the culture and society of Poland. This requires reflection on current practices and active thinking about what path needs to be followed to strive for this ideal. A full convergence of EU member states with the EU science-policy model may not be a desired outcome, but one can think of individual models that positively connect EU policies to science-policy models for individual countries, including a model for Poland. 


\section{REFERENCES}

Amekawa, Y., Sseguya, H., Onzere, S., and Carranza, I. (2010). Delineating the multifunctional role of agroecological practices: Toward sustainable livelihoods for smallholder farmers in developing countries. Journal of Sustainable Agriculture, 34(2), 202-228.

Aubin, D., and Varone, F. (2004). The evolution of European eater policy. In the evolution of national water regimes in Europe, edited by Ingrid Kissling-Näf and Stefan Kuks, 4986. Dordrecht: Kluwer Academic Publishers.

Balconi, M., Brusoni, S., and Orsenigo, L. (2010). In defence of the linear model: An essay. Research Policy, 39(1), 1-13.

Beck, S. (2011). Moving beyond the linear model of expertise? IPCC and the test of adaptation. Regional Environmental Change, 11(2), 297-306.

Beck, U. (1992). From industrial society to the risk society: questions of survival, social structure and ecological enlightenment. Theory, Culture and Society, 9(1), 97-123.

Behagel, J. (2012). The politics of democratic governance: the implementation of the Water Framework Directive in the Netherlands. PhD thesis. Wageningen: Wageningen University.

Behagel, J. H., Arts, B., and Turnhout, E. (2017). Beyond argumentation: a practice-based approach to environmental policy. Journal of Environmental Policy and Planning, 1-13.

Behagel, J., and Turnhout, E. (2011). Democratic legitimacy in the implementation of the Water Framework Directive in the Netherlands: Towards participatory and deliberative norms? Journal of Environmental Policy and Planning 13(3), 297-316.

Bergmann, M., Jahn, T., Knobloch, T., Krohn, W., Pohl, C., and Schramm, E. (2012). Methods for transdisciplinary research: a primer for practice. Campus Verlag.

Beyer, J. M. (1997). Research utilization: Bridging a cultural gap between communities. Journal of Management Inquiry, 6(1), 17-22.

Bhattacherjee, A. (2012). Social science research: Principles, methods, and practices. University of South Florida.

Bielak, A. T., Campbell, A., Pope, S., Schaefer, K., and Shaxson, L. (2008). From science communication to knowledge brokering: the shift from 'science push' to 'policy pull'. In Communicating science in social contexts: New Models, New Practices, edited by D. Cheng M. Claessens T. Gascoigne J. Metcalfe et al., (p. 201-226). Amsterdam: Springer.

Boari, C., and Riboldazzi, F. (2014). How knowledge brokers emerge and evolve: The role of actors' behaviour. Research Policy, 43, 683-695.

Boonstra, F.G. (2004). Laveren tussen regio's en regels. Verankering van beleidsarrangementen rond plattelandsontwikkeling in Noordwest Friesland, 
de Graafschap en Zuidwest Salland. PhD thesis, Nijmegen Catholic Univeristy. Assen. Uitgeverij Koninklijke Van Gorcum.

Borrass, L., Sotirov, M., and Winkel, G. (2015). Policy change and Europeanization: Implementing the European Union's habitats directive in Germany and the United Kingdom. Environmental Politics, 24(5), 788-809.

Börzel, T., and Buzogány, A. (2010). Environmental organisations and the Europeanisation of public policy in Central and Eastern Europe: the case of biodiversity governance. Environmental Politics, 19(5), 708-735.

Börzel, T.A. and Risse, T. (2003). Conceptualizing the domestic impact of Europe. In K. Featherstone and C., M. Radaelli (Eds.). The Politics of Europeanization. Oxford: Oxford University Press, 57-80.

Bouwma, I., Arts, B., and Liefferink, D. (2017). Cause, catalyst or conjunction? The influence of the Habitats Directive on policy instrument choice in Member States. Journal of Environmental Planning and Management, 60(6), 977-996.

Bradshaw, G. A., and Borchers, J. G. (2000). Uncertainty as information: narrowing the science-policy gap. Conservation Ecology, 4(1), [online] URL: http://www.consecol.org/vol4/iss1/art7/ .

Braun, M. (2016). Europeanization of environmental policy in the new Europe: Beyond conditionality. London: Routledge.

Bulmer, S. (2008). Theorizing Europeanization. In Europeanization edited by P. Graziano and M. Vink, 46-58. London: Palgrave Macmillan.

Burns, A. (2000). Facilitating collaborative action research: some insights from the AMEP. Prospect, 15(3), 23-34.

Bush, V. (1945). Science, the Endless Frontier: A Report to the President. US Govt. print. off. Washington.

Campbell, A. (2007). An investigation into the conservation impact of research published in the scientific literature. Doctoral dissertation, Faculty of Natural Science, Centre of Environmental Policy, Imperia College London.

Cantore, N., J. Kennan, S. Page and te Velde, D. W. (2011). Common Agricultural Policy (CAP) - reform and development. London, Overseas Development Institute, 52.

Carayannis, E., and Popescu, D. (2005). Profiling a methodology for economic growth and convergence: learning from the EU e-procurement experience for central and eastern European countries. Technovation, 25(1), 1-14.

Carlile, P. R. (2002). A pragmatic view of knowledge and boundaries: Boundary objects in new product development. Organization Science, 13(4), 442-455.

Carlile, P. (2004) Transferring, Translating, and Transforming: an Integrative Framework for Managing Knowledge Across Boundaries. Organization Science, 15, 555-568.

Carmin, J., and Vandeveer, S. D. (2004). Enlarging EU environments: Central and Eastern Europe from transition to accession. Environmental Politics, 13(1), 3-24. 
Carrozza, C. (2015). Democratizing expertise and environmental governance: Different approaches to the politics of science and their relevance for policy analysis. Journal of Environmental Policy and Planning, 17(1), 108-126.

Carter, C. (2013). Constructing sustainability in EU fisheries: Re-drawing the boundary between science and politics? Environmental Science and Policy, 30, 26-35.

Case-law of the Court of Justice, 2014. C-356/13. Judgment of the Court (Ninth Chamber) of 20 November 2014. European Commission v Republic of Poland.

Cash, D., Adger, W., Berkes, F., Garden, P., Lebel, L., Olsson, P., ... and Young, O. (2006). Scale and cross-scale dynamics: governance and information in a multilevel world. Ecology and Society, 11(2).

Cash, D., Clark, W., Alcock, F., Dickson, N. M., Eckley, N., Guston, D. H., ... and Mitchell, R. B. (2003). Knowledge systems for sustainable development. Proceedings of The National Academy of Sciences, 100(14), 8086-8091.

Cash, D., Clark, W., Alcock, F., Dickson, N., Eckley, N., and Jäger, J. (2002). Salience, credibility, legitimacy and boundaries: linking research, assessment and decision making. KSG Working Papers Series RWP02-046. Retrieved January 30, 2011, from http://ssrn.com/abstract=372280

Chapman, J. M., Algera, D., Dick, M., Hawkins, E. E., Lawrence, M. J., Lennox, R. J., ... and Vu, M. (2015). Being relevant: practical guidance for early career researchers interested in solving conservation problems. Global Ecology and Conservation, 4, 334-348.

Chilvers, J., and Kearnes, M. (Eds.). (2015). Remaking participation: Science, environment and emergent publics. London: Routledge.

Cianciara, A. K. (2017). Contestation of EU Climate Policy in Poland: Civil Society and Politics of National Interest. Prakseologia, 159, 237-264.

Clavel, L., Soudais, J., Baudet, D., and Leenhardt, D. (2011). Integrating expert knowledge and quantitative information for mapping cropping systems. Land Use Policy, 28(1), 57-65.

Cohen, L., and Manion, L. (1994). Research methods in education. (4th ed.) London: Routledge.

De Koning, J., Turnhout, E., Winkel, G., Blondet, M., Borras, L., Ferranti, F., ... and Jump, A. (2014). Managing climate change in conservation practice: an exploration of the science-management interface in beech forest management. Biodiversity and Conservation, 23(14), 3657-3671.

Demeritt, D. (2006). Science studies, climate change and the prospects for constructivist critique. Economy and Society, 35(3), 453-479.

Demmke, C., and Deakin, S. F. (2001). Towards effective environmental regulation: innovative approaches in implementing and enforcing European environmental law and policy. Cambridge, MA: Harvard Law School. 
Den Hertog, P. (2002). Co-producers of innovation: on the role of knowledge-intensive business services in innovation. Productivity, Innovation and Knowledge in Services, 223-255.

Denzin, N.K. (1989). The Research Act: A Theoretical Introduction to Sociological Methods. (3rd edition). New York: McGraw-Hill

Dilling, L., and Lemos, M. C. (2011). Creating usable science: Opportunities and constraints for climate knowledge use and their implications for science policy. Global Environmental Change, 21(2), 680-689.

Duina, F. (1997). Explaining legal implementation in the European Union. International Journal of the Sociology of Law, 25(2), 155-179.

Dunn, G., and Laing, M. (2017). Policy-makers perspectives on credibility, relevance and legitimacy (CRELE). Environmental Science and Policy, 76, 146-152.

Eden, S. (1996). Public participation in environmental policy: considering scientific, counterscientific and non-scientific contributions. Public Understanding of Science, 5(3), 183204. https://doi.org/10.1088/0963-6625/5/3/001

European Commission. 2010. Brochure about the Nitrites Directive. Retrieved from http://ec.europa.eu/environment/pubs/pdf/factsheets/nitrates.pdf

European Commission. (1991). Council Directive 91/676/EEC of 12 December 1991 Concerning the Protection of Waters Against Pollution Caused by Nitrates from Agricultural Sources. Brussels: European Commission.

European Commission. (2001). European Governance. A White Paper. COM(2001) 428 final. Brussels: European Commission.

European Commission. (2002). Communication from the Commission on the collection and use of expertise by the Commission: principles and guidelines. Improving the knowledge base for better policies. $\operatorname{COM(2002)~} 713$ final. Brussels: European Commission.

European Commission. (2003). Investing in Research: An action Plan for Europe, COM(2003) 226 final/2. Brussels: European Commission.

European Commission. (2010). Report from the Commission to the Council and the European Parliament on Implementation of Council Directive 91/676/EEC Concerning the Protection of Waters Against Pollution Caused by Nitrates from Agricultural Sources Based on Member States Reports. $\operatorname{COM(2010)~} 47$ final. Brussels: European Commission.

European Commission. (2013). Press Release: Environment: Commission takes Poland to Court Over Nitrates and Water Pollution. Brussels: European Commission. http://europa.eu/rapid/press-release_IP-13-48_en.htm

European Commission. (2013). Vademecum on public procurement in the Commission. European Commission, Budget, Central Financial Service. 
European Commission. (2016). Homepage of the European Commission. Retrieved December 2016, from http://ec.europa.eu/environment/water/waternitrates/index_en.html

European Commission. (2017). Communication from the Commission. EU law: Better results through better application. 2017/C18/02.Brussels: European Commission.

European Commission. (2018). Environment, the Nitrates Directive. Brussels: the European Commission. Retrieved September 29, 2018, from http://ec.europa.eu/environment/water/water-nitrates/index_en.html

European Commission. (2019). Homepage of the European Commission/Horizon 2020.

Retrieved January 2019 from https://ec.europa.eu/programmes/horizon2020/en/h2020section/responsible-research-innovation

Farrell, A. E., and Jäger, J. (Eds.). (2006). Assessments of regional and global environmental risks: designing processes for the effective use of science in decisionmaking. Resources for the Future.

Felt, U. (2017). "Response-able Practices" or "New Bureaucracies of Virtue": The Challenges of Making RRI Work in Academic Environments. In Responsible Innovation 3, 49-68. Springer, Cham.

Felt, U., Barben, D., Irwin, A., Joly, P. B., Rip, A., Stirling, A., and Stöckelová, T. (2013). Science in Society: caring for our futures in turbulent times. Policy Briefing, 50.

Felt, U., Wynne, B., Callon, M., Gonçalves, M. E., Jasanoff, S., and Jepsen, M. (2007). Taking European knowledge society seriously. Luxembourg: DG for Research. EUR, 22, 700.

Fernández, R. J. (2016). How to be a more effective environmental scientist in management and policy contexts. Environmental Science and Policy, 64, 171-176.

Fischer, F. (1990). Technocracy and the Politics of Expertise. SAGE Publications.

Fischer, F., and Forester, J. (Eds.). (1993). The argumentative turn in policy and planning. London: Duke University Press.

Fischer, F., and Gottweis, H. (2013). The argumentative turn in public policy revisited: twenty years later. Critical Policy Studies, 7(4), 425-433.

Flinders, M., and Buller, J. (2006). Depoliticisation: Principles, tactics and tools. British Politics, 1(3), 293-318.

Flyvbjerg, B. (2006). Five misunderstandings about case-study research. Qualitative Inquiry, 12(2), 219-245.

Frederiksen, P., van der Sluis, T., Vadineanu, A., Terkenli, T. S., Gaube, V., Busck, A. G., ... and Pedroli, B. (2017). Misfits and compliance patterns in the transposition and implementation of the Habitats Directive-four cases. Land Use Policy, 62, 337-350.

Funtowicz, S. (2006). Why knowledge assessment. Interfaces Between Science and Society, 1, 137-145.

Funtowicz, S. O., and Ravetz, J. R. (1993). The emergence of post-normal science. In Science, Politics and Morality, 85-123. Springer, Dordrecht. 
Funtowicz, S., and Ravetz, J. R. (1993). Science for the post-normal age. Futures, 25(7), 739755.

Garrard, G. E., Fidler, F., Wintle, B. C., Chee, Y. E., and Bekessy, S. A. (2016). Beyond advocacy: making space for conservation scientists in public debate. Conservation Letters, 9(3), 208-212.

Gaudreau, M., and Saner, M. (2014). Researchers are from Mars; Policymakers are from Venus. Policy Brief Series, Science/Policy Interface (1). Ottawa, Canada: University of Ottawa.

Gergen, K. J. (1985). The social constructionist movement in modern psychology, American Psychologist, 40, 266-275

Gershon, P. (1999). Review of civil procurement in central government. HM Treasury.

Gibbons, M. (1998). Higher Education Relevance in the 21st Century. Washington, DC.

Gibbons, M. (2000). Mode 2 society and the emergence of context-sensitive science. Science and Public Policy, 27(3), 159-163.

Gibbons, M., Limoges, C., Nowotny, H., Schwartzman, S., Scott, P., and Two, M. (1994). The new production of knowledge. The dynamics of science and research in contemporary societies. London: Sage.

Gieryn, T. F. (1983). Boundary-work and the demarcation of science from non-science: strains and interests in professional ideologies of scientists. American Sociological Review, 781-95.

Gilek, M., Udovyk, O., Karlsson, M., and Linke, S. (2016). Science and Policy in the Governance of Europe's Marine Environment: The impact of Europeanization, regionalization and the ecosystem approach to management. In Governing Europe's Marine Environment: Europeanization of Regional Seas or Regionalization of EU Policies, edited by Michael Gilek and Kristine Kern 141-163. Farnham: Ashgate Publishing.

Goffin, K., Koners, U., Baxter, D., and van der Hoven, C. (2010). Managing lessons learned and tacit knowledge in new product development. Research Technology Management, 53(4), 39-51.

Görg, C., H. Wittmer, Carter, C., Turnhout, E., VandeWalle, M., Schindler, S., and Livorell, B. (2016). Governance options for science-policy interfaces on biodiversity and ecosystem services: comparing a network versus a platform approach. Biodiversity and Conservation, 25, 1235-1252.

Gorton, M., Lowe, P., and Zellei, A. (2005). Pre-accession Europeanisation: The strategic realignment of the environmental policy systems of Lithuania, Poland and Slovakia towards agricultural pollution in preparation for EU Membership. Sociologia Ruralis, 45(3), 202-223.

Grimshaw, J. M., Eccles, M. P., Lavis, J. N., Hill, S. J., and Squires, J. E. (2012). Knowledge translation of research findings. Implementation Science, 7(1), 50. 
Hadorn, G. H., Biber-Klemm, S., Grossenbacher-Mansuy, W., Hoffmann-Riem, H., Joye, D., Pohl, C., ... and Zemp, E. (2008). The emergence of transdisciplinarity as a form of research. In Handbook of transdisciplinary research (19-39). Springer, Dordrecht.

Hajer, M., and Versteeg, W. (2005). A decade of discourse analysis of environmental politics: Achievements, challenges, perspectives. Journal of Environmental Policy and Planning, 7(3), 175-184.

Halffman, W. (2003). Boundaries of Regulatory Science. Boechout: Albatros.

Halffman, W. (2005). Science-policy boundaries: national styles? Science and Public Policy, $32(6), 457-467$.

Hallstrom, L. (2004). Eurocratising enlargement? EU elites and NGO participation in European environmental policy. Environmental Politics, 13, 175-196.

Haverland, M. (2000). National adaptation to European integration: The importance of institutional veto points. Journal of Public Policy, 20(1), 83-103.

Hegger, D., Lamers, M., Van Zeijl-Rozema, A., and Dieperink, C. (2012). Conceptualising joint knowledge production in regional climate change adaptation projects: success conditions and levers for action. Environmental Science and Policy, 18, 52-65.

Héritier, A., and Lehmkuhl, D. (2008). The shadow of hierarchy and new modes of governance. Journal of Public Policy, 28(1), 1-17.

Héritier, A., Knill, C., Douillet, A.-C., Kerwer, D., Lehmkuhl, D. and Teutsch, M. (2001). Differential Europe. The EU Impact on National Policy Making. Lanham, MD: Rowman and Littlefield.

Hessel, R., Van den Berg, J., Kaboré, O., Van Kekem, A., Verzandvoort, S., Dipama, J. M., and Diallo, B. (2009). Linking participatory and GIS-based land use planning methods: A case study from Burkina Faso. Land Use Policy, 26(4), 1162-1172.

Hisschemöller, M. (2004). Knowledge production and the limits of democracy. In Massen, S. and P. Weingart (Eds.). Democratization of Expertise? Exploring novel forms of scientific advice in political decision-making. Sociology of Sciences Yearbook 24. Dordrecht, Kluwer, 189-207.

Holmes, J., and Clark, R. (2008). Enhancing the use of science in environmental policymaking and regulation. Environmental Science and Policy, 11(8), 702-711.

Holmes, J., and Savgård, J. (2008). Dissemination and Implementation of Environmental Research: Including Guidelines for Best Practice. Naturvårdsverket.

Holzmann, V. (2013). A meta-analysis of brokering knowledge in project management. International Journal of Project Management, 31(1), 2-13.

Hoppe, R. (2010). From "knowledge use" towards "boundary work": sketch of an emerging new agenda for inquiry into science-policy interaction. Knowledge Democracy (p. 169186). Springer, Berlin, Heidelberg. 
Huitema, D., and Turnhout, E. (2009). Working at the science-policy interface: a discursive analysis of boundary work at the Netherlands Environmental Assessment Agency. Environmental Politics, 18(4), 576-594.

Hulme, M. (2010). Problems with making and governing global kinds of knowledge. Global Environmental Change, 20(4), 558-564.

Huntington, S. P. (1993). The clash of civilizations? Foreign Affairs, 22-49.

Igras, J., M. Fotyma, T. Jadczyszyn, W. Lipiński, and Radzimierski. M. (2008). Evaluation of the contamination of shallow ground waters, endangered directly by the inflow of biogenic substances particularly from agriculture and the potential impact of impurities from agricultural production on the environment. Puławy, Poland: Ministry of Agriculture and Rural Development.

Ingram, J., Mills, J., Dibari, C., Ferrise, R., Ghaley, B. B., Hansen, J. G., ... \& Molnar, A. (2016). Communicating soil carbon science to farmers: incorporating credibility, salience and legitimacy. Journal of Rural Studies, 48, 115-128.

Ishiyama, N. (2016). Role of knowledge brokers in communities of practice in Japan. Journal of Knowledge Management, 20(6), 1302-1317.

IZOO. ( $\left.\begin{array}{llll}2 & 0 & 1 & 2\end{array}\right)$. Oszacowanie wielkości projalubcaz jednostkowej zawartości azotu nawozów naturalnych, powstałych w rożnych systemach utrzymania zwierząt gospodarskich w Polsce. [Estimation of the Size of the Production Unit and the Nitrogen Content of Manure Produced in Different Animals' Housing Systems]. Balice, Poland: National Research Institute of Animal Production.

Jacob, M. (2006). Utilization of social science knowledge in science policy: Systems of Innovation, Triple Helix and VINNOVA. Social Science Information, 45(3), 431-462.

Jasanoff, S. (1990). The fifth branch: Science advisers as policymakers. Harvard University Press.

Jasanoff, S. (2000). Between risk and precaution-reassessing the future of GM crops. Journal of Risk Research, 3(3), 227-282.

Jasanoff, S. (2003). Accountability. (No?) Accounting for expertise. Science and Public Policy, $30(3)$, p. 157-162.

Jasanoff, S. (2005). Designs on nature: science and democracy in Europe and the United States. Princeton University Press.

Jasanoff, S. (2011). What is the regulatory science? Concept and history in United States and in Japan. Interview with Professor Sheila Jasanoff. Japanese translation published in Clinical Evaluation (39), 167-180.

Jasanoff, S. (Ed.). (2004). States of knowledge: the co-production of science and the social order. London: Routledge.

Jasanoff, S., Martello, M. L., and Haas, P. M. (2004). Earthly politics: local and global in environmental governance. MIT press. 
Jelonek, M., Keler, K., and Worek, B. (2010). Dane- ich gromadzenie, integracja i jakość. In Górniak, J.; and Mazur S. (red.), Polityki publiczne oparte na dowodach i ich zastosowanie do rynku pracy, Pracodawcy RP, (brak daty wydania, prawdopodobnie 2010 r.), Warszawa.

Johnston, E., and Soulsby, C. (2006). The role of science in environmental policy: an examination of the local context. Land Use Policy, 23(2), 161-169.

Jupp, V. (1996). Documents and critical research. In Sapsford R Jupp W (Eds.). Data collection and analysis. p. 298-316.London: Sage.

Kahn, H., and Wiener, A. J. (1967). The year 2000; a framework for speculation on the next thirty-three years. New York: Macmillan.

Karaczun, Z. (2005). Preparing for EU environmental policy in Poland: the case of the nitrates directive. Land Use Policy, 22(3), 245-253.

Kazancigil, A. (1998). Governance and science: market-like modes of managing society and producing knowledge. International Social Science Journal, 50(155), 69-79.

Kebo, S., and Saner, M. (2014). Making it Work: incentives to improve the science/policy interface. Workshop Backgrounder, Science/Policy Interface (4). Ottawa, Canada: University of Ottawa.

Kirchhoff, C. J., Lemos, M. C., and Dessai, S. (2013). Actionable knowledge for environmental decision making: broadening the usability of climate science. Annual Review of Environment and Resources, 38, 339-414.

Klein, J. T. (2004). Prospects for transdisciplinarity. Futures, 36(4), 515-526.

Klein, J. T., Grossenbacher-Mansuy, W., Häberli, R., Bill, A., Scholz, R. W., and Welti, M. (Eds.). (2001). Transdisciplinarity: joint problem solving among science, technology, and society: an effective way for managing complexity. Springer Science and Business Media.

Klijn, J.A.A., L.A.E. Vullinghs, M. v.d. Berg, H. van Meijl, R. van Lammeren, T. van Rheenen, A .A. Tabeau, A. Veldkamp, P.H. Verburg, H. Westhoek and Eickhout,B. (2005). The EURURALI S study: Technical document. Wageningen, Alterra, Alterra-rapport 1196.

Kluvánková-Oravská, T., Chobotová, V., Banaszak, I., Slavikova, L., and Trifunovova, S. (2009). From government to governance for biodiversity: the perspective of central and Eastern European transition countries. Environmental Policy and Governance, 19(3), 186-196.

Knight, A. T., Cowling, R. M., Rouget, M., Balmford, A., Lombard, A. T., and Campbell, B. M. (2008). Knowing but not doing: selecting priority conservation areas and the research-implementation gap. Conservation Biology, 22(3), 610-617.

Knill, C. (2001). The Europeanisation of national administrations: Patterns of institutional change and persistence. Cambridge University Press.

Knill, C. (2005). Introduction: Cross-national policy convergence: concepts, approaches and explanatory factors. Journal of European Public Policy 12(5), 764-74. 
Knill, C. and Lehmkuhl, D. (2002). The national impact of European Union regulatory policy: three Europeanization mechanisms. European Journal of Political Research 41(2), 25580 .

Knill, C., and Lehmkuhl, D. (1999). How Europe matters. Different mechanisms of Europeanization. European Integration Online Papers 3 (7). https://kops.unikonstanz.de/bitstream/handle/123456789/4271/1999_EIOP_Europeanization_Mec hansisms.pdf? sequence=1.

Knill, C., and Lenschow, A. (1998). Coping with Europe: the impact of British and German administrations on the implementation of EU environmental policy. Journal of European Public Policy, 5(4), 595-614.

Knill, C., and Lenschow, A. (2000). Implementing EU environmental policy: New directions and old problems. Manchester University Press.

Knill, C., and Tosun, J. (2009). Hierarchy, networks, or markets: how does the EU shape environmental policy adoptions within and beyond its borders? Journal of European Public Policy, 16(6), 873-894.

Knill, C., Tosun, J., and Bauer, M. W. (2009). Neglected faces of Europeanization: The differential impact of the EU on the dismantling and expansion of domestic policies. Public Administration, 87(3), 519-537.

Kok, K., Rothman, D. S., and Patel, M. (2006). Multi-scale narratives from an IA perspective: Part I. European and Mediterranean scenario development. Futures, 38(3), 261-284.

Koskinen, K., and Makinen, S. (2009). Role of boundary objects in negotiations of project contracts. International Journal of Project Management, 27, 31-38.

Kowalczewska, K., and Behagel, J. (2018). How policymakers' demands for usable knowledge shape science-policy relations in environmental policy in Poland. Science and Public Policy. https://doi.org/10.1093/scipol/scy065

Kowalczewska, K., and Turnhout, E. (2012). The Usability of Scenario Studies: the Case of the EUruralis from the Users' Perspective. Polish Sociological Review, (177), 91.

Kowalczewska, K., Behagel, J., and Turnhout, E. (2017). Infrastructures of expertise: policy convergence and the implementation of the EU Nitrates Directive in Poland. Journal of Environmental Planning and Management, 1-19.

Kraak, A. (2000). Changing modes: A brief overview of the mode 2 knowledge debate and its impact on South African policy formulation. Human Science Research. Pretoria, 1-37.

Kramer, J. M. (2004). EU enlargement and the Environment: Six Challenges. Environmental Politics, 13(1), 290-311.

Kružíková, E. ( $\left.\begin{array}{llll}2 & 0 & 0 & 4\end{array}\right)$. EU accession and legal change: accomplishments and challenges in the Czech case. Environmental Politics, 13(1), 99-113.

Kunseler, E. M., and Tuinstra, W. (2017). Navigating the authority paradox: Practicing objectivity in environmental expertise. Environmental Science and Policy, 67, 1-7. 
Kunseler, E. M., Tuinstra, W., Vasileiadou, E., and Petersen, A. C. (2015). The reflective futures practitioner: balancing salience, credibility and legitimacy in generating foresight knowledge with stakeholders. Futures, 66, 1-12.

Lahsen, M., Sanchez-Rodriguez, R., Lankao, P. R., Dube, P., Leemans, R., Gaffney, O., ... and Smith, M. S. (2010). Impacts, adaptation and vulnerability to global environmental change: challenges and pathways for an action-oriented research agenda for middleincome and low-income countries. Current Opinion in Environmental Sustainability, 2(5), 364-374.

Landry, R., Lamari, M., and Amara, N. (2003). The extent and determinants of the utilization of university research in government agencies. Public Administration Review, 63(2), 192-205.

Laurance, W. F., Koster, H., Grooten, M., Anderson, A. B., Zuidema, P. A., Zwick, S., ... and Anten, N. P. (2012). Making conservation research more relevant for conservation practitioners. Biological Conservation, 153, 164-168.

Lawton, J. H. (2007). Ecology, politics and policy. Journal of Applied Ecology, 44(3), 465-474.

Lemos, M. C., and Morehouse, B. J. (2005). The co-production of science and policy in integrated climate assessments. Global environmental change, 15(1), 57-68.

Lemos, M. C., Arnott, J. C., Ardoin, N. M., Baja, K., Bednarek, A. T., Dewulf, A., ... and Mach, K. J. (2018). To co-produce or not to co-produce. Nature Sustainability, 1(12), 722.

Lepori, B., Masso, J., Jabłecka, J., Sima, K., and Ukrainski, K. (2009). Comparing the organization of public research funding in central and eastern European countries. Science and Public Policy, 36(9), 667-681.

Leventon, J. (2015). Explaining implementation deficits through multi-level governance in the EU's new member states: EU limits for arsenic in drinking water in Hungary. Journal of Environmental Planning and Management, 58(7), 1137-1153.

Liefferink, D., Wiering, M., and Uitenboogaart, Y. (2011). The EU Water Framework Directive: A multi-dimensional analysis of implementation and domestic impact. Land Use Policy, 28(4), 712-722.

Lindblom, C., and Cohen, D. K. (1979). Usable knowledge. Social science and social problem solving. London: Yale University Press.

Locker, K. O. (1994). The challenge of interdisciplinary research', Journal of Business Communication, 31: 137-51.

Lomas, J. (2007). The in-between world of knowledge brokering. Bmj, 334(7585), 129-132.

Mastenbroek, E. 2005. EU compliance: Still a 'black hole? Journal of European Public Policy, 12(6), 1103-1120.

Matthews, A. (2010). How Might the EU's Common Agricultural Policy Affect Trade and Development After 2013? Issue Paper No. 29. ICTSD, Dublin, 15.

McCauley, D. (2008). Sustainable development and the 'governance challenge': the French experience with Natura 2000. European Environment, 18(3), 152-167. 
Meffe, G. K. (1998). Conservation scientists and the policy process. Conservation Biology, 12(4), 741-742.

Metze, T., and Turnhout, E. (2014). Politiek, participatie en experts in de besluitvorming over super wicked problems', Bestuurskunde, 23, 3-12.

Meyer, M. (2010). The rise of the knowledge broker. Science Communication, 32, 997-1011.

Michaels, S. (2009). Matching knowledge brokering strategies to environmental policy problems and settings. Environmental Science and Policy, 12, 994-1011.

Miller, C. A. (2008). Civic epistemologies: constituting knowledge and order in political communities. Sociology Compass, 2(6), 1896-1919.

Mills, T. J., and Clark, R. N. (2001). Roles of research scientists in natural resource decisionmaking. Forest Ecology and Management, 153(1-3), 189-198.

Milner-Gulland, E. J., Fisher, M., Browne, S., Redford, K. H., Spencer, M., and Sutherland, W. J. (2010). Do we need to develop a more relevant conservation literature? Oryx, 44(1), $1-2$.

Mocsári, J. (2004). Missing details behind the big picture: the delayed implementation of the Habitats Directive in Hungary. Budapest Papers on Europeanization, 18.

Munns, A. K., and Bjeirmi, B. F. (1996). The role of project management in achieving project success. International Journal of Project Management, 14(2), 81-87.

Muñoz-Erickson, T. A. (2014). Co-production of knowledge-action systems in urban sustainable governance: The KASA approach. Environmental Science and Policy, 37, 182-191.

Newig, J., and Fritsch, O. (2009). Environmental governance: participatory, multi-level-and effective? Environmental Policy and Governance, 19(3), 197-214.

Nilsson, M., Jordan, A., Turnpenny, J., Hertin, J., Nykvist, B., and Russel, D. (2008). The use and non-use of policy appraisal tools in public policy making: an analysis of three European countries and the European Union. Policy Sciences, 41(4), 335-355.

Nowotny, H., Scott, P., and Gibbons, M. (2003). Introduction: Mode 2'Revisited: The New Production of Knowledge. Minerva 41, 179-194

Nowotny, H., Scott, P., Gibbons, M., and Scott, P. B. (2001). Re-thinking science: Knowledge and the public in an age of uncertainty. Cambridge: Polity.

O'Donnell, P., and Deighton, B. (2015). A key element in research policy in Europe. Horizon Magazine (Special Issue March 2015). (doi 10.2777/95988)

Oldham, G., and McLean, R. (1997). Approaches to knowledge-brokering. International Institute for Sustainable Development paper, 23(06).

Olsen, J. P. (2002). The many faces of Europeanization. Journal of Common Market Studies, 40(5), 921-952.

Paloniemi, R., Apostolopoulou, E., Cent, J., Bormpoudakis, D., Scott, A., Grodzińska-Jurczak, M., ... and Pantis, J. D. (2015). Public participation and environmental justice in 
biodiversity governance in Finland, Greece, Poland and the UK. Environmental Policy and Governance, 25(5), 330-342.

Pielke Jr, R. A. (2007). The honest broker: making sense of science in policy and politics. Cambridge University Press.

Pohl, C. (2008). From science to policy through transdisciplinary research. Environmental Science and Policy, 11(1), 46-53.

Punch, K. F. (2005). Introduction to social research: Quantitative and qualitative approaches (2nd edition). London: Sage.

Radaelli, C. M. (1999). The public policy of the European Union: whither politics of expertise? Journal of European public policy, 6(5), 757-774.

Randhawa, K., Josserand, E., Schweitzer, J., \& Logue, D. (2017). Knowledge collaboration between organizations and online communities: the role of open innovation intermediaries. Journal of Knowledge Management, 21(6), 1293-1318.

Ravetz, J. R. (1990). The merger of knowledge with power: Essays in critical science. London: Mansell Publishing Limited.

Rayner, S. (2003). Democracy in the age of assessment: reflections on the roles of expertise and democracy in public-sector decision making. Science and Public Policy, 30(3), 163170.

Regeer, B. J., and Bunders, J. F. (2009). Knowledge co-creation: Interaction between science and society. A Transdisciplinary Approach to Complex Societal Issues. Den Haag: Advisory Council for Research on Spatial Planning, Nature and the Environment/Consultative Committee of Sector Councils in the Netherlands [RMNO/COS].

Renn, O. (1995). Style of using scientific expertise: a comparative framework. Science and Public Policy, 22(3), 147-156.

Rich, R. (1991). Knowledge Creation, Diffusion, and Utilization. Perspectives of the Founding Editor of Knowledge. Knowledge Creation, Diffusion, and Utilization 12 (3), 319-337.

Rienks,W., Hoek, S., Verweij, P., Lokers, R., and Vanmeulebrouk, B. (2008). Eururalis, a discussion support tool for rural Europe. iEMSs 2008: International Congress on Environmental Modelling and Software. Wageningen, The Netherlands: Wageningen University and Research.

Riesch, H. (2010). Theorizing boundary work as representation and identity. Journal for the Theory of Social Behaviour, 40(4), 452-473.

Rogowski, R. (1997). Rational choice as a Weberian view of culture. APSA-CP, 1997, 14-15.

Rolfstam, M. (2009). Public procurement as an innovation tool: the role of institutions. Science and Public Policy, 36(5), 349-360.

Saarela, S., Soderman, T., and Lyytimaki, J. (2015). Knowledge brokerage context factors What matters in knowledge exchange in impact assessment? Environmental Science and Policy 51 325-337. 
Sanderson, I. (2006). Complexity, "practical rationality" and evidence-based policy making. Policy and Politics, 34, 115-132.

Sarewitz, D. (2016). Saving science. The New Atlantis, 49, 4-40.

Sarewitz, D., and Pielke Jr, R. A. (2007). The neglected heart of science policy: reconciling supply of and demand for science. Environmental Science and Policy, 10(1), 5-16.

Sarkki, S., Niemelä, J., Tinch, R., Van Den Hove, S., Watt, A., and Young, J. (2013). Balancing credibility, relevance and legitimacy: a critical assessment of trade-offs in sciencepolicy interfaces. Science and Public Policy, 41(2), 194-206.

Sarkki, S., Tinch, R., Niemelä, J., Heink, U., Waylen, K., Timaeus, J., ... and van den Hove, S. (2015). Adding 'iterativity' to the credibility, relevance, legitimacy: a novel scheme to highlight dynamic aspects of science-policy interfaces. Environmental Science and Policy, 54, 505-512.

Saurugger, S. (2010). The social construction of the participatory turn: The emergence of a norm in the European Union. European Journal of Political Research, 49(4), 471-495.

Schure, J. M., and Arts, B. J. M. (2012). Common Agricultural Policy Reform and its Impact on Biodiversity and Livelihoods in Developing Countries-A review of scientific literature. Forest and Nature Conservation Policy Group, Wageningen University.

Schwandt, T. A. (1994). Constructivist, interpretivist approaches to human inquiry. In N. K. Denzin and Y. S. Lincoln (Eds.). Handbook of qualitative research (pp. 118-137). Thousand Oaks, CA, US: Sage Publications, Inc.

Shiers, D., Weston, J., Wilson, E., Glasson, J., and Deller, L. (2014). Implementing new EU environmental law: the short life of the UK Site Waste Management Plan Regulations. Journal of Environmental Planning and Management, 57(7), 1003-1022.

Shonkoff, J. P., and Bales, S. N. (2011). Science does not speak for itself: Translating child development research for the public and its policymakers. Child Development, 82(1), 17-32.

Small, S. A., and Uttal, L. (2005). Action-oriented research: Strategies for engaged scholarship. Journal of Marriage and Family, 67(4), 936-948.

Sotirov, M., Lovric, M., and Winkel, G. (2015). Symbolic transformation of environmental governance: implementation of EU biodiversity policy in Bulgaria and Croatia between Europeanization and domestic politics. Environment and Planning C: Government and Policy, 33(5), 986-1004.

Star, S. L., and Griesemer, J. R. (1989). Institutional ecology, translations' and boundary objects: Amateurs and professionals in Berkeley's Museum of Vertebrate Zoology, 1907-39. Social Studies of Science, 19(3), 387-420.

Sterk, B., Carberry, P., Leeuwis, C., Van Ittersum, M. K., Howden, M., Meinke, H., ... and Rossing, W. A. H. (2009). The interface between land use systems research and policy: multiple arrangements and leverages. Land Use Policy, 26(2), 434-442.

Stirling, A. (2006). Analysis, participation and power: justification and closure in participatory multi-criteria analysis. Land Use Policy, 23(1), 95-107. 
Stirling, A. (2006). From Science and Society to Science in Society: towards a framework for co-operative research. Project Report. European Commission, Directorate General for Research.

Sumner, J. (2003). Relations of suspicion: Critical theory and interdisciplinary research. History of Intellectual Culture, 3(1), 1-12.

Sverrisson, Á. (2001). Translation networks, knowledge brokers and novelty construction: Pragmatic environmentalism in Sweden. Acta Sociologica, 44(4), 313-327.

Tangney, P. (2017). What use is CRELE? A response to Dunn and Laing. Environmental Science and Policy, 77, 147-50.

The Eururalis homepage. (2011). Retrieved January 27, 2011, from http://www.eururalis.eu/index.htm.

Turnhout, E. (2003). Ecological indicators in Dutch nature conservation: science and policy intertwined in the classification and evaluation of nature. PhD thesis. Vrije Universitate Amsterdam, Aksant.

Turnhout, E. (2009). The effectiveness of boundary objects: the case of ecological indicators. Science and Public Policy, 36(5), 403-412.

Turnhout, E., Behagel, J., Ferranti, F., and Beunen, R. (2015). The construction of legitimacy in European nature policy: expertise and participation in the service of costeffectiveness. Environmental Politics, 24(3), 461-480

Turnhout, E., Hisschemöller, M., and Eijsackers, H. (2007). Ecological indicators: between the two fires of science and policy. Ecological Indicators, 7(2), 215-228.

Turnhout, E., Hisschemöller, M., and Eijsackers, H. (2008). Science in Wadden Sea policy: from accommodation to advocacy. Environmental Science and Policy, 11(3), 227-239.

Turnhout, E., Neves, K., and De Lijster, E. (2014). 'Measurementality' in biodiversity governance: knowledge, transparency, and the Intergovernmental Science-Policy Platform on Biodiversity and Ecosystem Services (IPBES). Environment and Planning A, 46(3), 581-597.

Turnhout, E., Stuiver, M., Klostermann, J., Harms, B., and Leeuwis, C. (2013). New roles of science in society: different repertoires of knowledge brokering. Science and Public Policy, 40(3), 354-365.

University of Warsaw. (2011). Wyznaczenie obszarów znajdujących się pod rzeczywista presją działalności rolniczej ze względu na zanieczyszczenie wód związkami azotu. [Designation of areas being under pressure of agriculture production in terms of nitrates pollution of waters]. Warsaw: University of Warsaw.

Valente, A., Castellani, T., Larsen, M., and Aro, A. R. (2014). Models and visions of sciencepolicy interaction: Remarks from a Delphi study in Italy. Science and Public Policy, $42(2), 228-241$.

Van Bommel, S. (2008). Understanding experts and expertise in different governance contexts. The case of nature conservation in the Drentsche Aa area in the Netherlands. $\mathrm{PhD}$ thesis. Wageningen: Wageningen University. 
Van den Hove, S. (2007). A rationale for science-policy interfaces. Futures, 39(7), 807-826.

Van der Zouwen, M. (2006). Nature Policy Between Trends and Traditions. Dynamics in nature policy arrangements in the Yorkshire Dales, Donana and the Veluwe. PhD thesis. Catholic University Nijmegen. Delft: Eburon

Van der Zouwen, M. W., and Top, M. (2000). European nature conservation in the Netherlands: a pioneer unable to implement its own ambitions. Radboud University Nijmegen.

Van Egmond, S., and Zeiss, R. (2010). Modeling for policy science-based models as performative boundary objects for Dutch policy making. Science and Technology Studies 23, 58-78.

Van Kammen, J., de Savigny, D., and Sewankambo, N. (2006). Using knowledge brokering to promote evidence-based policy-making: the need for support structures. Bulletin of the World Health Organization, 84 (8), 608-612.

Van Pelt, S. C., Haasnoot, M., Arts, B., Ludwig, F., Swart, R., and Biesbroek, R. (2015). Communicating climate (change) uncertainties: simulation games as boundary objects. Environmental Science and Policy, 45, 41-52.

Van Wyk, E., Breen, C. M., Sherwill, T., and Magadlela, D. (2007). Challenges for the relationship between science and society: developing capacity for ecosystem governance in an emerging democracy. Water Policy, 9(S2), 99-111.

Verboom, J., Alkemade, R., Klijn, J., Metzger, M. J., and Reijnen, R. (2007). Combining biodiversity modeling with political and economic development scenarios for $25 \mathrm{EU}$ countries. Ecological Economics, 62(2), 267-276.

Ward, V., House, A., and Hamer, S. (2009). Knowledge brokering: the missing link in the evidence to action chain? Evidence and Policy: a journal of research, debate and practice, 5(3), 267-279.

Wardekker, J. A., van der Sluijs, J. P., Janssen, P. H., Kloprogge, P., and Petersen, A. C. (2008). Uncertainty communication in environmental assessments: views from the Dutch science-policy interface. Environmental Science and Policy, 11(7), 627-641.

Waterton, C. (2002). From field to fantasy: classifying nature, constructing Europe. Social Studies of Science, 32(2), 177-204

Waterton, C., and Wynne, B. (1996). Building the European Union: science and the cultural dimensions of environmental policy. Journal of European Public Policy, 3(3), 421-440.

Wedeen, L. (2002). Conceptualizing culture: Possibilities for political science. American Political Science Review, 96(4), 713-728.

Weinberg, A. M. (1972). Science and trans-science. Minerva, 10(2), 209-222.

Weiss, C. H. (1979). The many meanings of research utilization. Public Administration Review, 39, 426-31.

Weiss, C. H. (1995). The haphazard connection: social science and public policy. International Journal of Educational Research, 23(2), 137-150. 
Wesselink, A., Buchanan, K. S., Georgiadou, Y., and Turnhout, E. (2013). Technical knowledge, discursive spaces and politics at the science-policy interface. Environmental Science and Policy, 30, 1-9.

Wildavsky, A. B. (1987). Speaking truth to power. Published by Transaction Publishers. New York.

Wilsdon, J., Doubleday, R., and Stirling, A. (2015). Future directions for scientific advice in Europe. Centre for Science and Policy.

Wooster, W. S. (1998). Science, advocacy, and credibility. Science, 282, 1823.

WUR. (2007). Environmental Sciences Wageningen University and Research Centre, November 2007. Contract 2006/441164/MAR/B1 (Implementation of the Nitrates Directive (91/676/EEC) Task 3. Wageningen: Wageningen University.

Wyborn, C. A. (2015). Connecting knowledge with action through coproductive capacities: adaptive governance and connectivity conservation. Ecology and Society, 20(1).

Yearley, S. (2004). Making sense of science: Understanding the social study of science. London: Sage.

Yin, R. K. (2017). Case study research and applications: Design and methods. Sage publications.

Young, J. C., Waylen, K. A., Sarkki, S., Albon, S., Bainbridge, I., Balian, E., ... and McCracken, D. (2014). Improving the science-policy dialogue to meet the challenges of biodiversity conservation: having conversations rather than talking at one-another. Biodiversity and Conservation, 23(2), 387-404.

Zybała, A. ( $\left.\begin{array}{llll}2 & 0 & 1 & 3\end{array}\right)$. Źródła wiedzy w politykach publicznych. Współczesna teoria i praktyka badań społecznych i humanistycznych", red. J. Juchnowski, R. Wiszniowski, Wrocław: Wydawnictwo Uniwersytetu Wrocławskiego.

Zybała, A. ( $\left.\begin{array}{llll}2 & 0 & 1 & 5\end{array}\right)$. Polityka publiczna w warunkach socjologicznej próżni. [Public policy in a socilogical vacuum]. Kultura i Społeczeństwo 4, 43-64.

Zybała, A. (2 0105 a). Polityka publiczna wobec teorii i jej praktyki w Polbteocławskie Studia Politologiczne, 18, 28-43. 


\section{SUMMARY}

The European Union (EU) plays an important role in steering the role of science in society in the direction of complex and interactive models of knowledge production that involve users and many other stakeholders. Many scholars emphasize that steering towards complex and interactive models of knowledge production and use is a current societal trend. Accordingly, these models are becoming part of social, cultural and political dynamics that cannot be separated from how we act in and organize the world. Important models include the model of Mode 2 knowledge production and use and the co-production model. These models are subject to normative and interventionist interpretations as they are turned into policy instruments and methods with the aim to improve science-policy and society relations and to effectively connect knowledge systems with user demands.

The influence of the EU affects science-policy relations in Poland. Poland is a country that has struggled to comply with EU environmental policy requirements and has already faced several legal procedures as a result. Poland is representative for many other Central and Eastern European (CEE) countries dealing with EU environmental policy, where national policy processes as well as science-policy relations today are to an important extent being steered by EU policy processes. This thesis examines how science-policy relations in environmental and rural development policy in Poland are being shaped by the EU and national processes. This includes an exploration of how policy demands that are articulated within EU and national processes affect science-policy relations and of the multiple directions in which these sciencepolicy relations change.

The main research question of the thesis asks how science-policy relations in environmental and rural development policy in Poland are shaped by EU policy processes. The research subquestions are:

A. How European and national policy cultures and institutions shape science-policy relations cultures and institutions shape science-policy relations?

B. How are science-policy interactions managed and/or steered in specific policy projects and programmes?

C. How do user and policy demands affect science-policy relations? 
The thesis is organized in six chapters. Chapters 2 to 5 present empirical studies, relate to research sub-questions, and are written as independent research articles for peer-reviewed journals. Chapter 6 concludes the thesis by answering the research questions and debating science-policy-society relations in Poland and the EU.

Chapter 2 addresses research sub-question A. It shows that the accession of Poland to the EU leads to a process of policy convergence in which member states' institutions and policy cultures become increasingly adapted to align with an EU governance system. Especially in EU environmental policy, knowledge and expertise are central to the institutions and policy cultures that are adapted in this process, which ideally results in the alignment of EU policy requirements with the administrative arrangements of member states. Chapter 2 offers a historical analysis of the Nitrates Directive's implementation in Poland and shows how increasing convergence of Polish institutions and cultures of expertise with EU policy occurred in response to the directive's requirements. This chapter highlights two important results: (1) knowledge and expertise are central to policy convergence processes and they are being stimulated by the top-down nature of EU environmental policy and Europeanisation processes in the case of Poland, and (2) institutions and cultures of expertise are entwined in 'infrastructures of expertise' and it is very hard to change one without the other. The chapter ends with a call for better consideration of the science-policy interface in Europeanisation processes.

Chapter 3 addresses research sub-question B. It shows how scenario studies can be useful tools to support planning and decision-making processes and how they play an important role in facilitating cooperation and interaction at the science-policy interface. It uses a theoretical framework that connects the criteria of credibility, salience and legitimacy to the concepts of co-production and boundary objects in order to analyse the EUruralis scenario study. EUruralis addresses the future of agriculture and rural development in Europe with four contrasting scenarios and colourful maps that are easily understandable for users. The findings show that EUruralis responded to the criteria of credibility, salience and legitimacy but different respondents, like policymakers and scientists, valued different things in EUruralis and their perception were very differentiated. The findings highlight that aspects related to legitimacy contributed to the capacity of EUruralis to function as a boundary object between the scientists and policymakers involved. The results also show how cooperation in the EUruralis 
project resulted in joint learning and reflection. The chapter concludes that if aspects related to legitimacy are taken into account while designing interactive and participatory processes of scenario studies, then they have a great potential to address complex issues and to stimulate reflection and co- production.

Chapter 4 also addresses research sub-question B. It discusses how the relation between science and policy is organized in one of the public procurement (PP) contracts which are commonly used by the European Commission to order scientific studies. By exploring the role of a knowledge broker in a real case setting and by using interviews to substantiate views and ideas, this chapter delivers evidence how a knowledge broker may ensure effectiveness of collaboration process between scientists and policymakers. This chapter also argues that PP offers a valuable contribution to science-policy interface studies because of its unique way to organize the relation between knowledge production and use, which is demand driven and guided by strict rules. The chapter presents knowledge brokering activities chose by a broker that are narrowed down to roles of intermediary and translator. The choice of the knowledge brokering activities was specific for this PP contract and is not easily transferable to other contexts. The knowledge broker used knowledge brokering activities related to the problem formulation, the identification of research needs, consulting with external stakeholders, and the management of the interactions between the procurer and the researcher. The role of a knowledge broker was played by a policymaker (representing the client of the public procurement contract). Policymakers are seen to have the main responsibility for the outcome of the scientific advice and the quality of the research process much depend on the clarity and quality of the policymakers in being able to identify and communicate about their policy needs and requirements. Therefore, this chapter showcases how policymakers can make a positive contribution to both knowledge production and use.

Chapter 5 address research sub-question C. It discusses how policymakers' demands for knowledge shape preferences for science-policy models such as Mode 1 and Mode 2. The chapter focuses on the demands that Polish policymakers make of science and how they envision their role in the knowledge production process in the field of environmental and rural development policy. In this chapter, a set of criteria on how policymakers define usable knowledge is applied to better understand preferences and uses-in-practice of different science-policy models. The results show that preferences for Mode 1 or Mode 2 depends on 
policy context and the particular usability criteria that are deemed important in that context. In the weighing of usability criteria, trade-offs appear to be inevitable, therefore preferences for science-policy models are in part the result of trade-off between criteria of quality, relevance, conformity, and action orientation. The findings also show that science can provide usable knowledge in both Mode 1 and 2. While Mode 2 may seem to be a better candidate to respond to multiple demands that policymakers make on the usability of knowledge, it often fails to make a convincing response to policymakers' need to base their decisions on knowledge that is considered objective. Therefore, Mode 1 remains attractive to policymakers when they have specific political demands: they may use it to avoid responsibility for negative policy outcomes or to discredit undesirable results. The chapter concludes it is likely that Mode 2 will not replace Mode 1 but work in tandem with it because policymakers are willing to use both science-policy models to tailor knowledge production processes to their demands for knowledge.

The concluding chapter of the thesis answers the main research question and sub-questions and reflects on the conceptual and methodological approaches taken in this thesis. These include debates on: i) the changing role of science and the shift from Mode 1 to Mode 2; ii) the imperfect shift towards Mode 2 in Poland; and iii) the role of knowledge and expertise in Europeanisation processes. Moreover, in the chapter I reflect on the case studies and my own role as a researcher as a active practitioner in these cases. The chapter concludes with recommendations for both policymakers and experts on how to manage science-policy relations. The recommendations highlight that while the EU has been searching for the rightful place of science in policy and society, there is a need to engage in a similar and strategic process about the ideal science-policy relation model in Poland that would connect with EU policies and at the same time best suit the culture and society of Poland. New societal demands put more attention to multi- and transdisciplinary research, involvement of nonscientific knowledge, engagement of users, and science communication. Scientists should be able to responds to these. Equally, policymakers need to take more responsibility for identifying and communicating what their policy needs and requirements towards science are. The quality of scientific advice also depends on the clarity and quality of the way in which demands and research needs are expressed and communicated. Coordinating and facilitating 
interactions in knowledge production and use processes is a role that both policymakers and scientists should be able to play. 


\section{ABOUT THE AUTHOR}

Katarzyna's research interest in the science-policy interface and the role of science in policymaking process in the field of environment and agriculture is strongly related to her professional career. She started her career with a short internship at Wageningen University and Research, where she had a lot of contacts with Dutch policymakers from the Ministry of Agriculture and where she learned how to develop a joint science-policy projects. She has

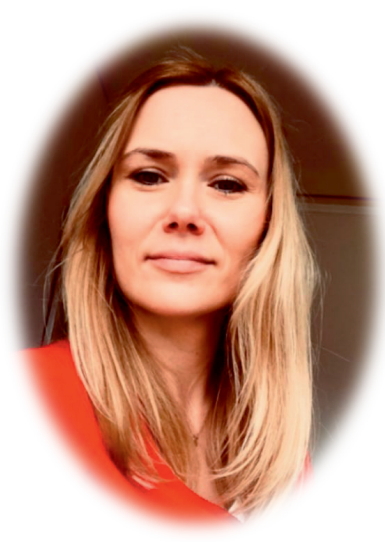
obtained a Master degree in Environmental Sciences of Wageningen University and a Master degree at Warsaw Agricultural University (Interfaculty Studies on Environmental Protection).

During and after her Master studies, Katarzyna worked for eight years at the Polish Ministry of Agriculture and Rural Development, being involved in analyses of the reforms of the Common Agriculture Policy and changes in agricultural sector due to various EU policies and global challenges. In 2012-2013, she was seconded to the European Commission, DG for Climate Action where she was responsible for actions related to mainstreaming climate objectives in the EU's rural development programmes, communicating of the Commission's proposal on Land Use, Land Use Change and Forestry (LULUCF) and LIFE climate action sub-programme.

The opportunity of working in different policy environments allowed Katarzyna to practice and experience with different policymaking models and science-policy relations. These experiences were an important input while writing this PhD thesis. In addition, they led to a change of her own cultural policy beliefs, where she increasingly came to acknowledge that different forms of close and interactive cooperation in knowledge production processes are conductive to effective environmental management and policies.

Currently, Katarzyna works at the Embassy of the Kingdom of the Netherlands on the position of agricultural policy advisor. She deals not only with policies but also with market and trade developments in the field of agriculture, nature, food quality, and climate change. Via embassy activities in Poland, she tries to promote different forms of cooperation, including publicprivate partnerships and multi-actor approaches by drawing on examples from the Netherlands. 
Cover design: by Aleksandra Jaczewska, Poland Printed by: ProefschriftMaken | Digiforce 